\title{
ON THE CORE OF A CONE-PRESERVING MAP
}

\author{
BIT-SHUN TAM AND HANS SCHNEIDER
}

\begin{abstract}
This is the third of a sequence of papers in an attempt to study the Perron-Frobenius theory of a nonnegative matrix and its generalizations from the cone-theoretic viewpoint. Our main object of interest here is the core of a cone-preserving map. If $A$ is an $n \times n$ real matrix which leaves invariant a proper cone $K$ in $\mathbb{R}^{n}$, then by the core of $A$ relative to $K$, denoted by $\operatorname{core}_{K}(A)$, we mean the convex cone $\bigcap_{i=1}^{\infty} A^{i} K$. It is shown that when core $_{K}(A)$ is polyhedral, which is the case whenever $K$ is, then $\operatorname{core}_{K}(A)$ is generated by the distinguished eigenvectors of positive powers of $A$. The important concept of a distinguished $A$-invariant face is introduced, which corresponds to the concept of a distinguished class in the nonnegative matrix case. We prove a significant theorem which describes a one-to-one correspondence between the distinguished $A$-invariant faces of $K$ and the cycles of the permutation induced by $A$ on the extreme rays of $\operatorname{core}_{K}(A)$, provided that the latter cone is nonzero, simplicial. By an interplay between cone-theoretic and graph-theoretic ideas, the extreme rays of the core of a nonnegative matrix are fully described. Characterizations of $K$-irreducibility or $K$-primitivity of $A$ are also found in terms of $\operatorname{core}_{K}(A)$. Several equivalent conditions are also given on a matrix with an invariant proper cone so that its spectral radius is an eigenvalue of index one. An equivalent condition in terms of the peripheral spectrum is also found on a real matrix $A$ with the Perron-Schaefer condition for which there exists a proper invariant cone $K$ such that $\operatorname{core}_{K}(A)$ is polyhedral, simplicial, or a single ray. A method of producing a large class of invariant proper cones for a matrix with the Perron-Schaefer condition is also offered.
\end{abstract}

\section{INTRODUCTION}

The classical theorems of Perron and Frobenius on spectral properties of nonnegative matrices have been studied and generalized in various ways. By now there is an extensive literature on the subject. In the book of Berman and Plemmons [B-P] the finite-dimensional aspects of this theory are described, whereas in the two monographs of Schaefer [Scha 1,2] the infinite-dimensional theory is developed. The past decade has witnessed a rapid development of the graph-theoretic spectral theory of nonnegative matrices (see [Schn 2, B-N,

Received by the editors December 3, 1991.

1980 Mathematics Subject Classification (1985 Revision). Primary 15A48, 47A10; Secondary 47H07, 93C05.

Key words and phrases. Cone-preserving map, core, distinguished eigenvalue, distinguished invariant face, nonnegative matrix, peripheral spectrum, Perron-Schaefer condition, polyhedral cone, simplicial cone, spectral radius of index one.

Research of the first author partially supported by the National Science Council of the Republic of China; the second author's research partially supported by NSF grants DMS 8901445 and ECS 871891. 
H-S 1, 2, 3] and the references therein) where emphasis is put on the relation between the combinatorial structure and the spectral structure of the generalized eigenspace associated with the spectral radius or distinguished eigenvalues of a nonnegative matrix. Many of the graph-theoretic ideas of a nonnegative matrix were also generalized to the integral operator setting (see [Nel, Vic 1, 2 and $\mathrm{J}-\mathrm{V}]$ ). The present authors are writing a sequence of (more or less independent, but related) papers [T-W, Tam 1, the present paper, T-S and Tam 2] in an attempt to study the Perron-Frobenius theory of a nonnegative matrix and its generalizations from the cone-theoretic viewpoint. This paper is the third in this sequence. At present our study is confined to the finite-dimensional setting. Our approach exploits the fact that a convex cone is a geometric object-a particular kind of convex sets; the facial and duality concepts play an important role in our treatment. In contrast to the previous treatments of the subject, especially in the infinite-dimensional cases, where function-theoretic methods dominated, our treatment is elementary, algebraic, geometric, and sometimes combinatorial in nature, more in the spirit of the paper [B-S]. We have found that many of the results about nonnegative matrices which were previously obtained by matrix-theoretic or combinatorial methods can also be proved by cone-theoretic methods. For instance, in [Tam 1] a simple cone-theoretic proof is offered to the Nonnegative-Basis Theorem for a singular $M$-matrix. Our approach appears to be quite promising; many fundamental interesting results are obtained which can clarify some known results, and may stimulate further investigations either in a finite-dimensional or in an infinite-dimensional setting. (The paper [T-W] has already led to the work of Friedland [Fri 1, 2] in the settings of a Banach space or a $C^{*}$-algebra.)

In the sequel our main object of interest is the core of a cone-preserving map. If $K$ is a proper (that is, closed, pointed, full convex) cone in a finitedimensional real vector space, and if $A$ is a linear mapping which takes $K$ into itself, then by the core of $A$ relative to $K$, which we denote by $\operatorname{core}_{K}(A)$, we mean the (convex) cone $\bigcap_{i=1}^{\infty} A^{i} K$. There are plausible reasons which explain why a study of the core of a cone-preserving map is worthwhile. First, in an initial study of the core of a nonnegative matrix (relative to the nonnegative orthant) Pullman [Pu 1] succeeded in rederiving the famous Frobenius theorem for an irreducible nonnegative matrix. This theorem, as we now know, is important to the treatment of nonnegative matrices by matrix-theoretic methods. Second, Birkhoff [Bir] gave an elementary proof of the Perron-Frobenius theorem for a cone-preserving map by considering the Jordan canonical form of a matrix. His method was later modified by Vandergraft [Van] to obtain an equivalent condition, now known as the Perron-Schaefer condition (which will be given in $\S 2$ ), for a matrix to have an invariant proper cone. Their proofs start by considering the limit of a convergent subsequence of $\left(A^{i} x /\left\|A^{i} x\right\|\right)_{i \in \mathbb{N}}$, where $A$ is the cone-preserving map under consideration and $x$ is an appropriate nonzero vector in the cone. But any such limit belongs to the core of $A$ (relative to the cone). So it seems likely that the core of $A$ contains much information about its spectral properties.

Hereafter in this section, unless specified otherwise, we always use $A$ to denote a linear mapping preserving some proper cone $K$ of $\mathbb{R}^{n}$. This paper is organised as follows. Background results and most of the necessary definitions 
are given in $\S 2$. In particular, we show that $\operatorname{core}_{K}(A)$ is always a closed, pointed cone mapped onto itself under $A$. In $\S 3$ we show that if $\operatorname{core}_{K}(A)$ is polyhedral, which is the case when $K$ is, then $\operatorname{core}_{K}(A)$ is generated by the distinguished eigenvectors (i.e. eigenvectors lying in the cone) of positive powers of $A$ corresponding to nonzero distinguished eigenvalues. The important concept of a distinguished $A$-invariant face of $K$ is introduced, which corresponds to the combinatorial concept of a distinguished class (introduced by Victory [Vic 3], related to the concept of a distinguished eigenvalue, and exploited in his work) in the nonnegative matrix case. Besides many other useful by-products, we prove a significant theorem (Theorem 3.14) which describes a one-to-one correspondence between the distinguished $A$-invariant faces of $K$ and the cycles of the permutation induced by $A$ on the extreme rays of core $_{K}(A)$, provided that the latter cone is nonzero, simplicial (which is the case, if $K$ is the nonnegative orthant and $A$ is a nonnilpotent nonnegative matrix). The relation between the $A$-invariant faces of $K$ and the spectral properties of $A$ will be investigated in our next paper [T-S].

In $\S 4$, where we apply our results of $\S 3$ to study the nonnegative matrix case, an interplay between cone-theoretic and graph-theoretic ideas dominates. The relation among the distinguished classes, the distinguished invariant faces of a nonnegative matrix and the cycles of the permutation induced by the matrix on the extreme rays of its core are clarified.

In $\S 5$ we characterize the $K$-primitivity or $K$-irreducibility of $A$ in terms of its core. The latter result extends a corresponding result of Pullman [Pul] on an irreducible nonnegative matrix. We also give several equivalent conditions on $A$ for its spectral radius to be an eigenvalue of index one, extending the work of Schaefer [Scha 3] on a nonnegative matrix.

Multipurpose examples illustrating how some of the results in the previous sections fail to hold when $\operatorname{core}_{K}(A)$ is not polyhedral or simplicial are given in $\S 6$. A subcone of $\operatorname{core}_{K}(A)$ which includes the cone generated by the distinguished eigenvectors of positive powers of $A$ corresponding to nonzero distinguished eigenvalues is also found. Our examples show that, in general, it is difficult to determine core $_{K}(A)$ completely, or to find an equivalent condition in terms of $A$ and $K$ for $\operatorname{core}_{K}(A)$ to be polyhedral or simplicial.

In $\S 7$ we solve completely the following problems: given an $n \times n$ real matrix $A$ that satisfies the Perron-Schaefer condition, find an equivalent condition on $A$ for which there exists a proper cone $K$ of $\mathbb{R}^{n}$ invariant under $A$ such that core $_{K}(A)$ is a polyhedral cone (a single ray, or a simplicial cone). It turns out that our conditions, like the Perron-Schaefer condition, are given in terms of the peripheral spectrum of $A$. In the infinite-dimensional settings, the peripheral point spectrum of a positive operator has been a source of interest, where the operator is usually assumed to be irreducible, or the underlying space is restricted to a special type, for instance, a Banach lattice (see [Scha 1, 2]). Our work may suggest further generalizations. In the course of establishing our results, we also develop a method of producing a large class of invariant proper cones for a matrix with the Perron-Schaefer condition. Conceivably, our method of construction will be useful not only to the study of the spectral properties of cone-preserving maps, but also to the study of allied fields, like linear dynamical systems, where invariant cones for matrices are often encountered (see, for instance, [B-N-S]). 
Finally, in $\S 8$ we indicate how one can carry over the results in this paper to the complex case.

\section{Preliminaries}

Unless stated otherwise, all matrices considered in this paper are square. We take for granted standard properties of nonnegative matrices, complex matrices and of graphs that can be found in many textbooks. A familiarity with elementary properties of finite-dimensional convex sets, convex cones and conepreserving maps is also assumed. To fix notation and terminology, we give some definitions.

Let $K$ be a nonempty subset of a finite-dimensional real vector space $V$. $K$ is called a convex cone if $\alpha x+\beta y \in K$ for all $x, y \in K$ and $\alpha, \beta \geq 0$. $K$ is pointed if $K \cap(-K)=\{0\} . K$ is full if its interior int $K$ (in the usual topology of $V$ ) is nonempty; equivalently, $K-K=V$. If $K$ is closed and satisfies all the above properties, $K$ is called a proper cone. Though our primary interest is on proper cones, closed, pointed cones occur frequently in our study. Hereafter in this section, we use $K$ to denote a closed, pointed cone in the $n$-dimensional euclidean space $\mathbb{R}^{n}$. By the dual cone of $K$, denoted by $K^{*}$, we mean the (closed) cone $\left\{z \in R^{n}:\langle z, x\rangle \geqq 0\right.$ for all $\left.x \in K\right\}$, where $\langle$, denotes the usual inner product of $\mathbb{R}^{n}$.

The cone $K$ induces a partial ordering on $\mathbb{R}^{n}$ by: $x \geqq^{K} y$ (also write as $y^{K} \leqq x$ ) if and only if $x-y \in K$. A subcone $F$ of $K$ is called a face of $K$ if $0^{K} \leqq y^{K} \leqq x$ and $x \in F$ imply $y \in F$. (Our definition of a face is related to the concept of an ideal in a partially ordered vector space as used by Bonsall [Bon] or an order ideal as in Ellis [Ell], and agrees with the usual definition of a face of a convex set, except that the empty set is not treated as a face of a cone.) If $S \subseteq K$, we denote by $\Phi(S)$ the face of $K$ generated by $S$, that is, the intersection of all faces of $K$ including $S$. If $x \in K$, we write $\Phi(\{x\})$ simply as $\Phi(x)$. For any face $F$ of $K$, we denote its relative interior and relative boundary (relative to its linear span) respectively by relint $F$ and $\operatorname{rbd} F$. If $x$ is a nonzero vector in $\mathbb{R}^{n}$, by the ray generated by $x$, which we denote by $\operatorname{ray}(x)$, we mean the set $\{\lambda x: \lambda \geqq 0\}$. Two nonzero vectors $x, y$ are said to be distinct if $\operatorname{ray}(x) \neq \operatorname{ray}(y)$. A vector $x \in K$ is called an extreme vector if either $x$ is the zero vector or $x$ is nonzero and $\Phi(x)=\operatorname{ray}(x)$; in the latter case, the face $\Phi(x)$ is called an extreme ray. $K$ is said to be polyhedral (or finitely generated, according to some authors) if it has finitely many extreme rays; it is called simplicial if it has exactly $m$ extreme rays, where $m$ is the dimension of $K$ (i.e. $\operatorname{dim} \operatorname{span} K$ ) .

$K$ is said to be the direct sum of its subcones $K_{1}, \ldots, K_{p}$, and we write $K=$ $K_{1} \oplus \cdots \oplus K_{p}$, if each vector in $K$ can be expressed uniquely as $x_{1}+x_{2}+\cdots+x_{p}$, where $x_{i} \in K_{i}, 1 \leqq i \leqq p . K$ is called decomposable if it is the direct sum of two nonzero subcones; otherwise, it is said to be indecomposable.

Let $C$ be a nonempty convex set in $\mathbb{R}^{n}$. We denote by $O^{+} C$ the set $\{y \in$ $\mathbb{R}^{n}: x+\lambda y \in C$ for every $\lambda \geqq 0$ and $\left.x \in C\right\}$; this set is a cone and is called the recession cone of $C$ [Roc, §8]. If, in addition, $C$ is closed, then the set $\left\{\alpha\left(\begin{array}{l}1 \\ x\end{array}\right): x \in C, \alpha \geqq 0\right\} \cup\left(\{0\} \times O^{+} C\right)$ is a closed cone of $\mathbb{R}^{n+1}$ [Roc, Theorem 8.2], referred to as the closed cone of $\mathbb{R}^{n+1}$ that arises from $C$ in the standard way. It is not difficult to prove that this latter cone is a proper cone of $\mathbb{R}^{n+1}$ 
if and only if the closed set $C$ has nonempty interior and contains no straight line.

We often use the terms "matrix" and "linear mapping" interchangeably. The nullspace and the range space of a matrix $A$ are denoted respectively by $\mathfrak{N}(A)$ and $\mathfrak{R}(A)$. When the nullspace or range space of a real matrix is considered, the subspace is usually meant to be taken in the corresponding real space. The spectrum of $A$ (in $\mathbb{C}$ ) is denoted by $\sigma(A)$, and its spectral radius by $\rho(A)$. Eigenvalues of $A$ with modulus $\rho(A)$ are said to compose the peripheral spectrum of $A$. By the index of $A$ we mean that smallest nonnegative integer $k$ such that $\operatorname{rank} A^{k}=\operatorname{rank} A^{k+1}$, and is denoted by $\nu(A)$. For any eigenvalue $\lambda$ of $A$. We denote by $\nu_{\lambda}(A)$ the index of $\lambda$ as an eigenvalue of $A$, that is, $\nu(A-\lambda I)$.

Let $A$ be an $n \times n$ real matrix. $K$ is said to be invariant under $A$, (or $A$ leaves invariant $K$ ) if $A K \subseteq K$. If $K$ is a proper cone of $\mathbb{R}^{n}$, we denote by $\pi(K)$ the set of all such matrices $A$. Matrices in $\pi(K)$ are referred to as cone-preserving maps (or more commonly as positive-operators on $K$ ).

Let $A \in \pi(K)$ (where $K$ is a proper cone). $A$ is said to be strictly $K$-positive if $A(K \backslash\{0\}) \subseteq$ int $K . A$ is said to be $K$-primitive if $A^{p}$ is strictly $K$-positive for some positive integer $p . A$ is said to be irreducible with respect to $K$, or called simply $K$-irreducible if the only faces of $K$ that it leaves invariant are $\{0\}$ and $K$ itself. By the index of imprimitivity of a $K$-irreducible matrix we mean the number of its distinct eigenvalues with modulus equal to its spectral radius.

We denote by $\mathbb{R}_{+}^{n}$ the nonnegative orthant of $\mathbb{R}^{n}$. Clearly $\pi\left(\mathbb{R}_{+}^{n}\right)$ is equal to the set of all $n \times n$ nonnegative matrices. Also when $K=\mathbb{R}_{+}^{n}$, the concepts of strict $K$-positivity, $K$-primitivity and $K$-irreducibility reduce respectively to the usual concepts of positivity, primitivity and irreducibility for nonnegative matrices.

A matrix $A \in \pi(K)$ is said to be an automorphism of $K$ if its inverse $A^{-1}$ exists and belongs to $\pi(K)$. The set of all automorphisms of $K$ forms a group under matrix multiplication and is denoted by $\operatorname{Aut}(K)$. It is clear that for any $n \times n$ real matrix $A, A \in \operatorname{Aut}(K)$ iff $A K=K$.

Let $A$ be an $n \times n$ real matrix. It is known that a necessary and sufficient condition for the existence of a proper cone $K$ of $\mathbb{R}^{n}$ such that $A \in \pi(K)$ is that the following conditions are satisfied:

(a) $\rho(A)$ is an eigenvalue of $A$.

(b) If $\lambda$ is an eigenvalue in the peripheral spectrum of $A$, then $\nu_{\lambda}(A) \leqq$ $\nu_{\rho(A)}(A)$.

The above conditions (a) and (b) together are now referred to as the PerronSchaefer condition (see [Schn 1, the paragraph after Theorem 1.1]). For more theorems of the above type, which relate the spectral properties and the geometric properties of a matrix, see [Dok; Els 1, 2; Schn 1; S-W 1, 2 and Van].

Let $A \in \pi(K)$. An eigenvalue $\lambda$ of $A$ is called a distinguished eigenvalue of $A$ for $K$ (or simply a distinguished eigenvalue of $A$, if there is no danger of confusion) if $A x=\lambda x$ for some nonzero vector $x \in K$; then $x$ is called a corresponding distinguished eigenvector. By the Perron-Frobenius theory for a cone-preserving map, $\rho(A)$ is always a distinguished eigenvalue of $A$. If $\lambda$ is a distinguished eigenvalue of $A$, then nonzero extreme vectors of the cone $\mathfrak{N}(\lambda I-A) \cap K$ (the cone of distinguished eigenvectors of $A$ corresponding to the eigenvalue $\lambda$ ) are called extremal distinguished eigenvectors of $A$. 
We now collect the necessary graph-theoretic definitions. For reference, see [Schn 2].

Let $P$ be an $n \times n$ nonnegative matrix. Denote by $\langle n\rangle$ the set $\{1, \ldots, n\}$. As usual, we define the directed graph of $P$ to be the graph $G(P)$ with vertex set $\langle n\rangle$ where $(i, j)$ is an arc if and only if $p_{i j} \neq 0$. The vertex sets of the strongly connected components of $G(P)$ are called simply classes of $P$, and are denoted by Greek letters $\alpha, \beta$, etc. For any two classes $\alpha, \beta$ of $P$, we say $\alpha$ has access to $\beta$, or $\beta$ has access from $\alpha$, if either $\alpha=\beta$ or there is a path in $G(P)$ from a vertex in $\alpha$ to some vertex in $\beta$. Two classes of $P$ are said to be noncomparable if they have no access to each other. Accessibility relations between vertices of $G(P)$ are also defined in a similar way. We also say a vertex $i$ has access to a class $\alpha$ with the obvious meaning. A class $\alpha$ of $P$ is said to be distinguished if $\rho\left(P_{\alpha \alpha}\right)>\rho\left(P_{\beta \beta}\right)$ for any class $\beta$ which has access to $\alpha$ but not equal to $\alpha$, where we use $P_{\alpha \alpha}$ to denoted the (principal) submatrix of $P$ associated with the class $\alpha$. A class $\alpha$ is said to be basic if $\rho\left(P_{\alpha \alpha}\right)=\rho(P)$.

The following theorem which was implicit in [Frob] and proved by Victory [Vic 3] (see the paragraph preceding Theorem 3.7 in [Schn 2]) relates the concept of a distinguished eigenvalue to the concept of a distinguished class for a nonnegative matrix. We shall make use of this result several times, and refer to it as the Frobenius-Victory Theorem. We have also found a cone-theoretic proof and a generalization of this result. For the details, see [T-S].

Theorem 2.1. Let $P$ be an $n \times n$ nonnegative matrix.

(i) For any real number $\lambda, \lambda$ is a distinguished eigenvalue of $P\left(\right.$ for $\left.\mathbb{R}_{+}^{n}\right)$ if and only if there exists a distinguished class $\alpha$ of $P$ such that $\rho\left(P_{\alpha \alpha}\right)=\lambda$.

(ii) If $\alpha$ is a distinguished class of $P$, then there is a (up to multiple) unique distinguished (i.e. nonnegative) eigenvector $x^{\alpha}=\left(\xi_{1}, \ldots, \xi_{n}\right)^{T}$ corresponding to $\rho\left(P_{\alpha \alpha}\right)$ with the property that $\xi_{i}>0$ if and only if $i$ has access to $\alpha$.

(iii) For each distinguished eigenvalue $\lambda$ of $P$, the cone $\mathfrak{N}(\lambda I-P) \cap \mathbb{R}^{n}$ is simplicial, its extreme vectors being the distinguished eigenvectors $x^{\alpha}$ of $P$ associated with its distinguished classes $\alpha$ such that $\rho\left(P_{\alpha \alpha}\right)=\lambda$ as given in (ii).

If $\alpha$ is a distinguished class of a nonnegative matrix $P$, we shall call $\alpha$ a distinguished class for the eigenvalue $\rho\left(P_{\alpha \alpha}\right)$. The original form of the theorem as stated by Victory [Vic 3] contains only parts (i) and (ii). Part (iii) is essentially due to Schneider [Schn 2, Theorem 3.7]. But we have added the observation that the (distinct) extremal distinguished eigenvectors of $P$ corresponding to the eigenvalue $\lambda$ are linearly independent. This follows from the combinatorial properties of the supports of these vectors.

Let $A$ be an $n \times n$ real matrix, and let $K$ be a closed, pointed cone of $\mathbb{R}^{n}$ invariant under $A$. We shall denote by $\operatorname{core}_{K}(A)$ the core of $A$ relative to $K$, i.e. the cone $\bigcap_{i=1}^{\infty} A^{i} K$. When there is no dander of confusion, we shall write it simply as core $(A)$.

The first attempt to study the core of a cone-preserving map was made by Pullman [Pul]. His work was mainly concerned with the core of an irreducible nonnegative matrix, but he also did some initial work on the core of a linear mapping that leaves invariant a polyhedral cone. The following result about the core of a general cone-preserving map is known (see [B-P, Chapter 1, Exercise 5.16]). For completeness, we offer a simple proof here. 
Theorem 2.2. Let $K$ be a proper cone. If $A \in \pi(K)$ then $\operatorname{core}(A)$ is a closed, pointed cone and $\left.A\right|_{\text {span(core }(A))} \in \operatorname{Aut}(\operatorname{core}(A))$.

Proof. First of all, for any positive integer $p$, note that we can write $\operatorname{core}(A)$ as $\bigcap_{i=p}^{\infty} A^{i} K$, since $\left(A^{i} K\right)_{i \in \mathbb{N}}$ forms a descending sequence under inclusion. For simplicity, write $\nu(A)$ as $\nu$. It is clear that $\operatorname{core}(A)$ is a pointed cone. To establish the closedness of $\operatorname{core}(A)$, denote by $K_{1}$ the closed, pointed cone $\mathfrak{R}\left(A^{\nu}\right) \cap K$. Then for each $i=0,1, \ldots, A^{i} K \supseteq A^{i} K_{1} \supseteq A^{i+\nu} K$, and hence we have, $\operatorname{core}_{K}(A)=\operatorname{core}_{K_{1}}(A)$. As the restriction map $\left.A\right|_{\mathscr{N}\left(A^{\nu}\right)}$ is nonsingular and $K_{1} \subseteq \mathfrak{R}\left(A^{\nu}\right), A^{i} K_{1}$ is closed for $i=1,2, \ldots$; thus the closedness of core $_{K_{1}}(A)$ and hence that of core $K_{K}(A)$ follows. Since $\left.A\right|_{\mathscr{R}\left(A^{\nu}\right)}$ is one-to-one, we also have, $A[\operatorname{core}(A)]=A\left(\bigcap_{i=\nu}^{\infty} A^{i} K\right)=\bigcap_{i=\nu}^{\infty} A^{i+1} K=\operatorname{core}(A)$.

We can readily obtain the following

Corollary 2.3. Let $K$ be a proper cone. If $A \in \pi(K)$ then $\operatorname{core}(A)$ is the largest subcone of $K$ such that the restriction of $A$ to its linear span is an automorphism.

The following result is due to Pullman [Pul]. His proof depends on a compactness argument and invokes the use of a separation theorem for convex sets. For the details, refer to Theorem 2.1 there.

Theorem 2.4. Let $K$ be a proper polyhedral cone. If $A \in \pi(K)$ then $\operatorname{core}(A)$ is a polyhedral cone whose number of extreme rays does not exceed that of $K$.

It is clear that the result of Theorem 2.2 (as well as those of Corollary 2.3 and Theorem 2.4) still holds if $K$ is taken to be a closed, pointed cone invariant under $A$.

\section{POlyhedral CORE}

We first make an easy observation:

Let $A \in \pi(K)$ where $K$ is a proper cone. Then $\operatorname{core}(A)$ is the zero cone if and only if $A$ is nilpotent.

The "if" part of this result is obvious. The "only if" part follows from the Perron-Frobenius theorem for a cone-preserving map: if $\rho(A)>0$, the corresponding distinguished eigenvector of $A$ belongs to $\operatorname{core}(A)$. So, henceforth, when we consider a cone-preserving map we often tacitly assume that its spectral radius is positive.

If $A \in \pi(K)$, where $K$ is a proper cone, it is obvious that every distinguished eigenvector of $A$ (or, of its positive powers) that corresponds to a nonzero distinguished eigenvalue belongs to $\operatorname{core}(A)$. We shall denote by $D_{k}(A)$ (or simply by $D_{k}$, if there is no danger of confusion) the cone generated by the distinguished eigenvectors of $A^{k}$ corresponds to its nonzero distinguished eigenvalues. Then clearly $D_{k} \subseteq \operatorname{core}(A)$. Also we have the following result:

Lemma 3.1. Let $K$ be a proper cone, and let $A \in \pi(K)$. Then each of the following holds:

(i) For each positive integer $k, A D_{k}=D_{k}$.

(ii) For any positive integers $i, j, D_{i} \subseteq D_{j}$ if $j$ is a multiple of $i$. 
(iii) If $A$ has a nonzero distinguished eigenvalue other than $\rho(A)$, then for each positive integer $k, D_{k}$ is a decomposable cone.

(iv) $\bigcup_{i=1}^{\infty} D_{i}$ is included in $\operatorname{core}(A)$, and is equal to $D_{k}$ for some positive integer $k$.

Proof. The proofs of (i), (ii) are easy.

(iii): Under that given assumption, for any positive integer $k, A^{k}$ has a nonzero distinguished eigenvalue other than $\rho(A)^{k}$. But, as can be shown, $D_{k}=\bigoplus\left[\mathfrak{N}\left(\lambda I-A^{k}\right) \cap K\right]$, where the direct sum is taken over all nonzero distinguished eigenvalues $\lambda$ of $A^{k}$ (see, for instance, the proof of Lemma 5.3 in [Hor]), so $D_{k}$ admits a nontrivial direct decomposition.

(iv): It is obvious that $\bigcup_{i=1}^{\infty} D_{i} \subseteq \operatorname{core}(A)$. To prove the second part of (iv), we assume that the contrary holds. Then for each positive integer $k$, we can find a positive integer $p$ such that $D_{p} \not \subset D_{k}$; hence, by the result of part (ii) $D_{k}$ is strictly included in $D_{k p}$. It follows that we can find a strict ascending sequence $D_{k_{1}} \subset D_{k_{2}} \subset D_{k_{3}} \subset \cdots$ such that for each $i, k_{i}$ divides $k_{i+1}$. Let us examine the implication of a strict inclusion between $D_{k_{i}}$ and $D_{k_{i+1}}$. Denote by $\mu_{1}, \ldots, \mu_{q}$ all the distinct nonzero distinguished eigenvalues of $A^{k_{i}}$. Then $D_{k_{i}}=\bigoplus_{j=1}^{q}\left[\mathfrak{N}\left(\mu_{j} I-A^{k_{i}}\right) \cap K\right]$. Suppose that $k_{i+1}=p k_{i}$. Then $\mu_{1}^{p}, \ldots, \mu_{q}^{p}$ are distinct nonzero distinguished eigenvalues of $A^{k_{i+1}}$ and $\mathfrak{N}\left(\mu_{j} I-A^{k_{i}}\right) \subseteq \mathfrak{N}\left(\mu_{j}^{p} I-A^{k_{i+1}}\right)$ for $j=1, \ldots, q$. In order that $D_{k_{i}}$ is strictly included in $D_{k_{i+1}}$, either $A^{k_{i+1}}$ has a nonzero distinguished eigenvalue distinct from $\mu_{1}^{p}, \ldots, \mu_{q}^{p}$, or for some $j=1, \ldots, q$,

$$
\operatorname{dim} \mathfrak{N}\left(\mu_{j} I-A^{k_{i}}\right)<\operatorname{dim} \mathfrak{N}\left(\mu_{j}^{p} I-A^{k_{i+1}}\right) .
$$

So passing from $A^{k_{i}}$ to $A^{k_{i+1}}$, we have either an increase in the number of distinct nonzero distinguished eigenvalues, or an increase in the dimension of an eigenspace corresponding to a nonzero distinguished eigenvalue. But the number of distinct eigenvalues, and the dimensions of the eigenspaces are all bounded above by the dimension of $K$; so the existence of the above strict ascending sequence $D_{k_{1}} \subset D_{k_{2}} \subset \cdots$ is impossible.

Now we restrict our attention to the case when $\operatorname{core}_{K}(A)$ is a polyhedral cone. According to Theorem 2.4 this covers the important case when $K$ is a polyhedral cone, and hence also the nonnegative matrix case. By Theorem 2.2 $A$ is an automorphism of $\operatorname{core}(A)$ when restricted to its linear span. So $A$ maps the set of extreme rays of $\operatorname{core}(A)$ one-to-one onto itself; hence $A$ permutes the extreme rays of its core. We shall denote the induced permutation by $\tau_{A}$. By the order of $\tau_{A}$ we mean, as usual, the smallest positive integer $m$ such that $\tau_{A}^{m}$ is the identity permutation. As a permutation $\tau_{A}$ can be written as a composition of unique (up to the ordering) disjoint cycles. If $\sigma$ is one such cycle, then by abuse of language we shall refer to the extreme rays of $\operatorname{core}(A)$ which are not fixed by $\sigma$ as extreme rays in the cycle $\sigma$.

Theorem 3.2. Let $K$ be a proper cone, and let $A \in \pi(K)$. Suppose that $\operatorname{core}(A)$ is nonzero, polyhedral. Let $m$ be the order of the permutation $\tau_{A}$. Then for each positive integer $k$, we have, $D_{k}=\operatorname{core}(A)$ if and only if $k$ is a multiple of $m$.

Proof. "If" part: As mentioned before, we always have, $D_{k} \subseteq \operatorname{core}(A)$. If $x$ is an extreme vector of $\operatorname{core}(A)$, then since $\tau_{A}^{m}$ is the identity permutation, we 
have $A^{m} x=\lambda x$ for some positive scalar $\lambda$ (depending on $x$ ); hence $x \in D_{m}$, and so $x \in D_{k}$ for any positive multiple $k$ of $m$. Since this is true for each extreme vector $x$ of core $(A)$, it follows that whenever $k$ is a multiple of $m$, we have, $\operatorname{core}(A) \subseteq D_{k}$ and hence $\operatorname{core}(A)=D_{k}$.

"Only if" part: Suppose that $D_{k}=\operatorname{core}(A)$. Then since

$$
D_{k}=\bigoplus\left[\mathfrak{N}\left(\lambda I-A^{k}\right) \cap K\right],
$$

where the direct sum is taken over all nonzero distinguished eigenvalues $\lambda$ of $A^{k}$, each nonzero extreme vector of $\operatorname{core}(A)$ is an extremal distinguished eigenvector of $A^{k}$ corresponding to a nonzero eigenvalue. It follows that $\tau_{A}^{k}$ is the identity permutation, and so $k$ is a multiple of $m$.

Corollary 3.3. Let $K$ be a proper cone, and let $A \in \pi(K)$.

(i) Suppose that $\operatorname{core}(A)$ is polyhedral. If there exists a positive integer $k$ such that $A^{k}$ has a nonzero distinguished eigenvalue other than $\rho\left(A^{k}\right)$, then $\operatorname{core}(A)$ is a decomposable cone.

(ii) $\operatorname{core}(A)$ is a simplicial cone if $K=\mathbb{R}_{+}^{n}$ (or, is a simplicial cone).

Proof. (i): It is clear that $\operatorname{core}\left(A^{k}\right)=\operatorname{core}(A)$. If $t$ is the order of the permutation induced by $A^{k}$ on the extreme rays of its core, then by Theorem 3.2, we have, $\operatorname{core}\left(A^{k}\right)=D_{t}\left(A^{k}\right)$. Since $A^{k}$ has a nonzero distinguished eigenvalue other than $\rho\left(A^{k}\right)$, by Lemma 3.1(iii) $D_{t}\left(A^{k}\right)$, and hence core $(A)$, is a decomposable cone.

(ii): Suppose that $K=\mathbb{R}_{+}^{n}$. Then $A$ is a nonnegative matrix. Let $m$ denote the order of the permutation $\tau_{A}$. Then we have $\operatorname{core}(A)=D_{m}=$ $\bigoplus\left[\mathfrak{N}\left(\lambda I-A^{m}\right) \cap \mathbb{R}_{+}^{n}\right]$, where the direct sum is taken over all nonzero distinguished eigenvalues $\lambda$ of $A^{m}$. But by the Frobenius-Victory Theorem (Theorem 2.1), for each nonzero distinguished eigenvalue $\lambda$ of $A^{m}, \mathfrak{N}\left(\lambda I-A^{m}\right) \cap \mathbb{R}_{+}^{n}$ is a simplicial cone; hence, $\operatorname{core}(A)$ is a simplicial cone.

Corollary 3.4. Let $K$ be a proper cone, and let $A \in \pi(K)$. If $\operatorname{core}(A)$ is polyhedral, then in $\operatorname{core}(A)$ there is no generalized eigenvectors of any positive powers of $A$ other than eigenvectors.

Proof. It suffices to prove our assertion for $A$, since $A$ and its positive powers have the same core relative to $K$. From Theorem $3.2 \mathrm{span}(\operatorname{core}(A))$ has a basis consisted of (distinguished) eigenvectors of $A^{m}$, where $m$ is the order of the permutation $\tau_{A}$. So the restriction map $\left(\left.A\right|_{\operatorname{span}(\operatorname{core}(A))}\right)^{m}$ is diagonalizable, and hence so is $\left.A\right|_{\operatorname{span}(\operatorname{core}(A))}$, since the latter linear map is nonsingular (see [L-T, Theorem 9.4.7]). It follows that in $\operatorname{core}(A)$ there is no generalized eigenvectors of $A$ of order two or more.

Theorem 3.5. Let $K$ be a proper polyhedral cone. If $A \in \operatorname{Aut}(K)$ then $A$ is diagonalizable (over $\mathbb{C})$.

Proof. If $A \in \operatorname{Aut}(K)$, then $\operatorname{core}_{K}(A)=K$. So our assertion follows from the proof of Corollary 3.4.

We still assume that $\operatorname{core}_{K}(A)$ is a polyhedral cone. As a permutation $\tau_{A}$ distributes the extreme rays of $\operatorname{core}(A)$ into various (disjoint) cycles. As noted by Pullman [Pul] there is a useful connection between these cycles and the nonzero distinguished eigenvalues of $A$ : Suppose that $\sigma$ is one such cycle of length $d$. Choose a nonzero vector, say $x$, from one of the extreme rays in the 
cycle. Since $A^{d}$ mays $\operatorname{ray}(x)$ onto itself, there exists a positive number $\lambda$ such that $A^{d} x=\lambda^{d} x$. Let $v=\sum_{i=0}^{d-1} \lambda^{-i} A^{i} x$. Then $v$ is a nonzero vector of $K$, since each of the vectors $A^{i} x, 0 \leqq i \leqq d-1$, is a nonzero vector in $K$ and $K$ is a pointed cone. A straightforward computation shows that $A v=\lambda v$. Hence $v$ is a distinguished eigenvector of $A$ corresponding to the nonzero eigenvalue $\lambda$. If we have chosen a different vector $x$ from either the same ray or from a different ray in the cycle, the resulting vector $v$ would have differed only by a positive multiple. We shall call $v$ the distinguished eigenvector of $A$ associated with the cycle $\sigma$ for the eigenvalue $\lambda$.

If a cycle $\sigma$ of $\tau_{A}$ contains a ray, $\operatorname{ray}(x)$, such that $x$ is a distinguished eigenvector of $A^{k}$ corresponding to the distinguished eigenvalue $\lambda^{k}$, where $\lambda>0$, then it is easy to show that every nonzero vector in each ray of $\sigma$ is also a distinguished eigenvector of $A^{k}$ corresponding to $\lambda^{k}$; furthermore, the distinguished eigenvector of $A$ associated with $\sigma$ is for the eigenvalue $\lambda$ (and $k$ is a multiple of the length of $\sigma$ ).

The above argument can also be used to establish the following result (in which no polyhedrality assumption is made on core $(A))$.

Lemma 3.6. Let $A \in \pi(K)$ where $K$ is a proper cone. Let $k$ be a positive integer. Then for any $\lambda \geqq 0, \lambda$ is a distinguished eigenvalue of $A$ if and only if $\lambda^{k}$ is a distinguished eigenvalue of $A^{k}$.

We now introduce a new concept: a distinguished face for a cone-preserving map, which corresponds to the concept of a distinguished class in the nonnegative matrix case (see Lemma 4.1). Let $K$ be a proper cone, and let $A \in \pi(K)$. A face $F$ of $K$ is said to be $A$-invariant if $A F \subseteq F ; F$ is said to be distinguished $A$-invariant (for the eigenvalue $\rho_{F}$ ) if $F$ is nonzero $A$-invariant, and for any nonzero $A$-invariant face $G$ properly included in $F$, we have, $\rho_{G}<\rho_{F}$, where we use $\rho_{F}$ to denote the spectral radius of the restriction map $\left.A\right|_{\text {span } F}$. We often call a distinguished $A$-invariant face simply a distinguished face. Note that if $F$ is a distinguished face, the eigenvector of $A$ corresponding to $\rho_{F}$ must lie in relint $F$. Also this eigenvector is (up to multiples) the only eigenvector of $A$ in $F$ that corresponds to $\rho_{F}$, and hence is an extremal distinguished eigenvector of $A$. So it is clear that a face is distinguished if and only if it is generated by an extremal distinguished eigenvector.

We shall need the following general results.

Lemma 3.7. Let $K$ be a proper cone, and let $A \in \operatorname{Aut}(K)$. If $F$ is an $A$ invariant face of $K$, then $A F=F$.

Proof. By hypothesis, $A F \subseteq F$. If the equality does not hold, then there exists a vector $x \in F$ whose (unique) pre-image (in $K$ ) under $A$, say $y$, does not lie in $F$. Then $\operatorname{span} F+\operatorname{span} \Phi(y)$ is a subspace properly including $\operatorname{span} F$ such that $A[\operatorname{span} F+\operatorname{span} \Phi(y)] \subseteq \operatorname{span} F$. This contradicts the fact that $A$ is nonsingular.

Lemma 3.8. Let $K$ be a proper cone, and let $A \in \pi(K)$. Let $F$ be an $A$ invariant face of $K$. Then $\operatorname{core}_{F}(A)$ is equal to $\operatorname{core}_{K}(A) \cap F$, and is in fact an $A$-invariant face of $\operatorname{core}_{K}(A)$. 
Proof. It is straightforward to verify that core $_{K}(A) \cap F$ is an $A$-invariant face of $\operatorname{core}_{K}(A)$. Since $\left.A\right|_{\text {spancore }(A)} \in \operatorname{Aut}(\operatorname{core}(A))$, by Lemma 3.7, we have,

$$
\begin{aligned}
\operatorname{core}_{K}(A) \cap F & =A\left[\operatorname{core}_{K}(A) \cap F\right]=\bigcap_{i=1}^{\infty} A^{i}\left[\operatorname{core}_{K}(A) \cap F\right] \\
& \subseteq \bigcap_{i=1}^{\infty} A^{i}(F)=\operatorname{core}_{F}(A) .
\end{aligned}
$$

But the reversed inclusion $\operatorname{core}_{F}(A) \subseteq \operatorname{core}_{K}(A) \cap F$ is obvious, so the equality holds.

If $A$ is an $n \times n$ real matrix and $\mu$ is a nonreal complex eigenvalue, then we call the subspace $\mathbb{R}^{n} \cap[\mathfrak{N}(\mu I-A) \oplus \mathfrak{N}(\bar{\mu} I-A)]$ the real eigenspace of $A$ corresponding to the conjugate pair $\mu, \bar{\mu}$. Similarly, we define the real generalized eigenspace of $A$ corresponding to the conjugate pair $\mu, \bar{\mu}$ to be $\mathbb{R}^{n} \cap\left[\mathfrak{N}\left((\mu I-A)^{m}\right) \oplus \mathfrak{N}\left((\bar{\mu} I-A)^{m}\right)\right]$ where $m=\nu_{\mu}(A)$. (Alternatively, one can also define the real eigenspace and the real generalized eigenspace to be respectively $\mathfrak{N}\left(\left(a^{2}+b^{2}\right) I-2 a I+A^{2}\right)$ and $\mathfrak{N}\left(\left[\left(a^{2}+b^{2}\right) I-2 a I+A^{2}\right]^{m}\right)$, where $a, b \in \mathbb{R}, b \neq 0$ such that $\mu=a+i b$. For references, see [B-N-S] or [G-L-R].) For convenience, we will say $M$ is the direct sum of the (real) eigenspaces (resp. generalized eigenspaces) of a real matrix $A$ corresponding to eigenvalues with certain properties to mean that it is the direct sum of the eigenspaces (resp. generalized eigenspaces) of $A$ corresponding to real eigenvalues and the real eigenspaces (resp. real generalized eigenspaces) corresponding to conjugate pair of complex eigenvalues, where all eigenvalues to be concerned have the given properties.

Theorem 3.9. Let $K$ be a proper cone of $\mathbb{R}^{n}$, and let $A \in \pi(K)$ with $\rho(A)>0$. Suppose that $\rho(A)$ is an eigenvalue of $A$ of index one. Let $M($ resp. $N)$ be the direct sum of the (real) eigenspaces (resp. (real) generalized eigenspaces) of $A$ corresponding to eigenvalues with modulus $\rho(A)$ (resp. with modulus less than $\rho(A))$. Denote by $P$ the projection of $\mathbb{R}^{n}$ onto $M$ along $N$. Then each of the following holds:

(i) There exists a subsequence of $\left((A / \rho(A))^{k}\right)_{k \in \mathbb{N}}$ which converges to $P$. Hence $P \in \pi(K)$.

(ii) $M=\operatorname{span}(M \cap K)$.

(iii) $M \cap K \subseteq \operatorname{core}(A)$.

(iv) The peripheral spectrum of $A$ and that of the restriction of $A$ to $\operatorname{span}(\operatorname{core}(A))$ are the same, counting algebraic multiplicities.

Proof. Note that since the eigenvalue $\rho(A)$ is of index one, by the PerronSchaefer condition, all eigenvalues in the peripheral spectrum of $A$ are of index one; hence $\mathbb{R}^{n}=M \oplus N$ and $P$ is a well-defined projection. To establish our result we need only consider the special case when $A$ is in a real canonical form. (This observation has also been exploited by Stern and Wolkowicz in their paper [S-W 1].) Also, we may, without loss of generality, assume that $\rho(A)=1$.

(i): We may assume that

$$
A=\operatorname{diag}\left(I_{p},-I_{q}, D_{1}, \ldots, D_{k}, B\right)
$$

where $D_{i}(i=1, \ldots, k)$ are real $2 \times 2$ matrices of the form

$$
\left[\begin{array}{cc}
\cos \theta_{i} & \sin \theta_{i} \\
-\sin \theta_{i} & \cos \theta_{i}
\end{array}\right],
$$


in which $\theta_{i} \neq t \pi$ for any integer $t, \rho(B)<1$ and, except for the identity block $I_{p}$, the blocks may or may not appear. By a well-known result in number theory about the simultaneous approximation of real numbers by rational numbers (see, for instance, [H-W, p. 170, Theorem 201]), given any $\varepsilon>0$, we can find a positive integer $q$ such that for every $i, 0 \leqq i \leqq k, q \theta_{i} / 2 \pi$ differs from an integer by less than $\varepsilon$, where we have set $\bar{\theta}_{0}$ to be $\pi$ (to take into account the possible eigenvalue -1$)$. It follows that we can find positive integers $q_{1}<q_{2}<\cdots$ such that the sequence $\left(A^{q_{i}}\right)_{i \in \mathbb{N}}$ converges to the projection $P$, and hence $P \in \pi(K)$.

(ii): Since $K$ is a full cone of $\mathbb{R}^{n}$ and $P$ is a projection onto $M$, clearly $P K$ is a full cone of $M$. But as proved in part (i) $P \in \pi(K)$, so $P K$ is included in $M \cap K$. (In fact, the equality holds.) Hence, we have $M=\operatorname{span}(M \cap K)$.

(iii): By the result of part (i), there exists a sequence $\left(q_{i}\right)_{i \in \mathbb{N}}$ of natural numbers such that $P=\lim _{i \rightarrow \infty} A^{q_{i}}$. So it is clear that $M \subseteq \mathfrak{R}\left(A^{\nu(A)}\right)$. Let $K_{1}$ denote the cone $\mathfrak{R}\left(A^{\nu(A)}\right) \cap K$. Since $A$ is nonsingular when restricted to $\mathfrak{R}\left(A^{\nu(A)}\right), A^{i} K_{1}$ is closed for every positive integer $i$. Choose any vector $x \in M \cap K$. Then $x \in K_{1}$, and we have

$$
x=P x=\lim _{i \rightarrow \infty} A^{q_{i}} x \in \bigcap_{i=1}^{\infty} \mathrm{cl} A^{q_{i}} K_{1}=\bigcap_{i=1}^{\infty} A^{q_{i}} K_{1}=\operatorname{core}_{K_{1}}(A) .
$$

But from the proof of Theorem 2.2 the last set is equal to $\operatorname{core}(A)$. So our assertion holds.

(iv): Since $\operatorname{core}(A)$ contains an eigenvector of $A$ corresponding to $\rho(A)$, it is clear that $\rho\left(\left.A\right|_{\mathrm{span}(\operatorname{core}(A))}\right)=\rho(A)$. By the results of parts (ii) and (iii), $M=\operatorname{span}(M \cap K) \subseteq \operatorname{span}(\operatorname{core}(A))$. So by the definition of $M$, it follows that the linear mappings $A$ and $\left.A\right|_{\text {span(core }(A))}$ have the same eigenvalues in their peripheral spectra, counting geometric multiplicities, which in this case are the same as algebraic multiplicities.

A modification of the proof of part (iii) of Theorem 3.9 yields the following:

Remark 3.10. Let $A \in \pi(K)$ where $K$ is a proper cone. If $y$ is a vector in $K$ which is the limit of some convergent subsequence of a sequence of the form $\left(x_{i}\right)_{i \in \mathbb{N}}$, with $x_{i} \in A^{i} K$ for each $i$, then $y \in \operatorname{core}_{K}(A)$.

Corollary 3.11. Let $K$ be a proper cone, and let $A \in \pi(K)$. Suppose that core $(A)$ is a polyhedral cone. If the index of $\rho(A)$ as an eigenvalue of $A$ is one, then every eigenvalue in the peripheral spectrum of $A$ is equal to $\rho(A)$ times a root of unity.

Proof. It is known that if $B$ is a linear mapping that leaves invariant a proper polyhedral cone, then every eigenvalue in the peripheral spectrum of $B$ is equal to $\rho(B)$ times a root of unity (see [B-T, Theorem 2]; also [Tam 1, Theorem 7.6] for a correction of the proof). So by part (iv) of Theorem 3.9 our assertion follows.

It is natural to ask of the relation between the $A$-invariant faces of $K$ and those of $\operatorname{core}_{K}(A)$, and in particular between their distinguished $A$-invariant faces. Denote by $\mathfrak{F}(K)$ the set of all faces of $K$, and also by $\mathfrak{F}_{A}(K)$ the set of all $A$-invariant faces of $K$. As can be easily shown, the association $F \mapsto \operatorname{core}_{K}(A) \cap F$ is a well-defined mapping from $\mathfrak{F}(K)$ to $\mathfrak{F}\left(\operatorname{core}_{K}(A)\right)$, 
sending $A$-invariant faces to $A$-invariant faces. Similarly, the association $G \mapsto$ $\Phi(G)$ is also a well-defined mapping from $\mathfrak{F}\left(\operatorname{core}_{K}(A)\right)$ to $\mathfrak{F}(K)$, sending $A$ invariant faces to $A$-invariant faces. In the following example we show that both mappings are in general not one-to-one, nor onto, and also that the first mapping may not send distinguished faces to distinguished faces. However, after giving the example, we shall show that the second mapping always induces a bijection between the distinguished faces of $\operatorname{core}_{K}(A)$ and those of $K$ for nonzero distinguished eigenvalues.

Example 3.12. Let $K_{1}=\operatorname{pos}\left\{e_{1}, e_{2}-e_{1}\right\}$, where we use $\operatorname{pos}(S)$ to denote the positive hull of the set $S$, and $e_{1}, e_{2}$ to denote the standard unit vectors of $\mathbb{R}^{2}$. Also let $A=\operatorname{diag}(1,2)$. Then $A \in \pi\left(K_{1}\right)$ and core $K_{1}(A)=\mathbb{R}_{+}^{2}$. Note that $K_{1}$ has exactly two nonzero $A$-invariant faces, namely, $K_{1}$ itself and $\Phi\left(e_{1}\right)$, both being distinguished faces. On the other hand, core $K_{1}(A)$ has three nonzero $A$-invariant faces, namely, $\operatorname{core}_{K_{1}}(A), \operatorname{ray}\left(e_{1}\right)$ and $\operatorname{ray}\left(e_{2}\right)$. The mapping $F \mapsto$ core $_{K_{1}}(A) \cap F$ from $\mathfrak{F}_{A}\left(K_{1}\right)$ to $\mathfrak{F}_{A}\left(\operatorname{core}_{K_{1}}(A)\right)$ is not onto, as $\operatorname{ray}\left(e_{2}\right)$ has no preimage. Note also that, whereas $K_{1}$ is a distinguished face of itself, $\operatorname{core}_{K_{1}}(A) \cap$ $K_{1}=\operatorname{core}_{K_{1}}(A)$ is not a distinguished face of $\operatorname{core}_{K_{1}}(A)$, as $\rho_{\text {core }_{K_{1}}(A)}=\rho_{\text {ray }\left(e_{2}\right)}=$ 2 . On the other hand, the mapping $G \mapsto \Phi(G)$ from $\mathfrak{F}_{A}\left(\operatorname{core}_{K_{1}}(A)\right)$ to $\mathfrak{F}_{A}\left(K_{1}\right)$ is not one-to-one, because we have $\Phi\left(\operatorname{ray}\left(e_{2}\right)\right)=\Phi\left(\right.$ core $\left._{K_{1}}(A)\right)=K_{1}$.

Next, consider $K_{2}=\operatorname{pos}\left\{e_{2}-e_{1}, e_{2}\right\}$. With the same $A$ as above, clearly $A \in$ $\pi\left(K_{2}\right)$ and core $_{K_{2}}(A)=\operatorname{ray}\left(e_{2}\right)$. In this case, the mapping $F \mapsto \operatorname{core}_{K_{2}}(A) \cap F$ from $\mathfrak{F}_{A}\left(K_{2}\right)$ to $\mathfrak{F}_{A}\left(\right.$ core $\left._{K_{2}}(A)\right)$ is not one-to-one, as $K_{2} \cap\left(\operatorname{core}_{K_{2}}(A)\right)$ and $\Phi\left(e_{2}\right) \cap$ core $_{K_{2}}(A)$ are both equal to $\operatorname{ray}\left(e_{2}\right)$, whereas the mapping $G \mapsto \Phi(G)$ from $\mathfrak{F}_{A}\left(\operatorname{core}_{K_{2}}(A)\right)$ to $\mathfrak{F}_{A}\left(K_{2}\right)$ is not onto, as $K_{2}$ has no pre-image.

Theorem 3.13. Let $A \in \pi(K)$. Suppose that $\operatorname{core}_{K}(A)$ is a nonzero cone. Then the association $G \mapsto \Phi(G)$ gives a one-to-one correspondence between the set of distinguished $A$-invariant faces of $\operatorname{core}_{K}(A)$ and the set of distinguished $A$ invariant faces of $K$ for nonzero distinguished eigenvalues.

Proof. Let $G$ be a distinguished face of $\operatorname{core}_{K}(A)$. By Theorem 2.2 and Lemma 3.7 we have $A G=G$; hence $\rho_{G}>0$. Let $x$ be the unique eigenvector of $A$ corresponding to $\rho_{G}$ that lies in $G$. Then $x \in$ relint $G$, and hence $\widetilde{\Phi}(x)=$ $G$ and $\Phi(G)=\Phi(x)$, where we use $\widetilde{\Phi}(x)$ to denote the face of $\operatorname{core}_{K}(A)$ generated by $x$ (and, as before, use $\Phi(S)$ to denote the face of $K$ generated by $S$ ). Note that $\Phi(x)$ cannot contain an eigenvector of $A$, distinct from $x$, that corresponds to $\rho_{G}$, because any such vector necessarily belongs to $\operatorname{core}_{K}(A)$ (as $\rho_{G}>0$ ) and hence lies in $G$, which contradicts the above uniqueness assumption on $x$. It follows that $\Phi(G)$ is a distinguished face of $K$. This proves that the assumption $G \mapsto \Phi(G)$ is a well-defined mapping from the set of distinguished faces of $\operatorname{core}_{K}(A)$ to the set of distinguished faces of $K$ for nonzero distinguished eigenvalues.

Let $G_{1}, G_{2}$ be distinguished $A$-invariant faces of $\operatorname{core}_{K}(A)$ such that $\Phi\left(G_{1}\right)$ $=\Phi\left(G_{2}\right)$. Then $G_{i}, i=1,2$, contains a unique eigenvector of $A$ corresponding to $\rho_{G_{i}}$, say, $x_{i}$. From our preceding proof, we have, $G_{i}=\widetilde{\Phi}\left(x_{i}\right), \Phi\left(G_{i}\right)$ is a distinguished face of $K$ and $x_{i}$ is the corresponding unique distinguished eigenvector of $A$ that lies in its relative interior. But now $\Phi\left(G_{1}\right)=\Phi\left(G_{2}\right)$, so $x_{1}=x_{2}$, and hence $G_{1}=\widetilde{\Phi}\left(x_{1}\right)=\widetilde{\Phi}\left(x_{2}\right)=G_{2}$. This proves that the mapping $G \mapsto \Phi(G)$ is one-to-one. 
If $F$ is a distinguished face of $K$ such that $\rho_{F}>0$, and if $x$ is the corresponding distinguished eigenvector of $A$ that lies in relint $F$, then it is not difficult to show that $\widetilde{\Phi}(x)$ is a distinguished face of $\operatorname{core}_{K}(A)$ such that $\Phi(\widetilde{\Phi}(x))=F$. This proves that the mapping $G \mapsto \Phi(G)$ is onto.

Now we come to the main theorem of this section.

Theorem 3.14. Let $K$ be a proper cone, and let $A \in \pi(K)$. Suppose that core $_{K}(A)$ is a nonzero, simplicial cone. For each cycle $\sigma$ of $\tau_{A}$ we denote by $F_{\sigma}$ the face of $K$ generated by the distinguished eigenvector of $A$ associated with $\sigma$. Then we have

(i) The association $\sigma \mapsto F_{\sigma}$ is a one-to-one correspondence between the set of cycles of $\tau_{A}$ and the set of distinguished A-invariant faces of $K$ for nonzero distinguished eigenvalues.

(ii) For any cycle $\sigma$ of $\tau_{A}$, the eigenvalues in the peripheral spectrum of $\left.A\right|_{\text {span } F_{\sigma}}$ are simple, and are exactly $\rho_{F_{\sigma}}$ times all the $d_{\sigma}$ th roots of unity, where $d_{\sigma}$ is the length of $\sigma$.

Proof. (i): Suppose that $\tau_{A}$ is the product of disjoint cycles $\sigma_{1}, \ldots, \sigma_{k}$. For each $i, 1 \leqq i \leqq k$, denote by $v_{i}$ the distinguished eigenvector of $A$ associated with the cycle $\sigma_{i}$. Since core $_{K}(A)$ is simplicial, it is not difficult to see that $\operatorname{core}_{K}(A)$ is a direct sum of $\widetilde{\Phi}\left(v_{1}\right), \ldots, \widetilde{\Phi}\left(v_{k}\right)$, where $\widetilde{\Phi}\left(v_{i}\right)$ denotes the face of $\operatorname{core}_{K}(A)$ generated by $v_{i}$, and that $\widetilde{\Phi}\left(v_{i}\right), 1 \leqq i \leqq k$, are all the distinguished faces of core $_{K}(A)$. Hence $\sigma_{i} \mapsto \widetilde{\Phi}\left(v_{i}\right)$ gives a one-to-one correspondence between the set of cycles of $\tau_{A}$ and the set of distinguished faces of core $_{K}(A)$. On the other hand, by Theorem $3.13 \widetilde{\Phi}\left(v_{i}\right) \mapsto \Phi\left(\widetilde{\Phi}\left(v_{i}\right)\right)$ gives a one-to-one correspondence between the set of distinguished faces of $\operatorname{core}_{K}(A)$ and the set of distinguished faces of $K$ for nonzero distinguished eigenvalues. But $\Phi\left(\widetilde{\Phi}\left(v_{i}\right)\right)=\Phi\left(v_{i}\right)=F_{\sigma_{i}}$, so our assertion follows.

(ii): Denote by $v$ the distinguished eigenvector of $A$ associated with the cycle $\sigma$ of $\tau_{A}$. By Lemma 3.8 and the above proof of part (i), $\operatorname{core}_{F_{\sigma}}(A)=$ core $_{K}(A) \cap F_{\sigma}=$ core $_{K}(A) \cap \Phi(v)$ and is an $A$-invariant face of core ${ }_{K}(A)$, which clearly includes the face $\widetilde{\Phi}(v)$. Now the index of $\rho_{F_{\sigma}}$ as an eigenvalue of $A$ is equal to one, as $A$ has an eigenvector, namely $v$, that lies in relint $F_{\sigma}$. Hence by Theorem 3.9(iv) the peripheral spectrum of $\left.A\right|_{\operatorname{span} F_{\sigma}}$ and that of the restriction of $A$ to $\operatorname{span}\left(\operatorname{core}_{F_{\sigma}}(A)\right)$ are the same, counting algebraic multiplicities. Note that as a simplicial cone $\operatorname{core}_{K}(A) \cap \Phi(v)$ is a direct sum of the face $\widetilde{\Phi}(v)$ and its complementary face $G$, both of which are $A$-invariant. Also we have $\rho\left(\left.A\right|_{\text {span } G}\right)<\rho_{F_{\sigma}}$; otherwise, $G$, and hence $\Phi(v)$ would contain a distinguished eigenvector of $A$ distinct from $v$ that corresponds to $\rho_{F_{\sigma}}$, contradicting the already proved fact that $F_{\sigma} \quad(=\Phi(v))$ is a distinguished face of $K$. Hence the peripheral spectrum of the restriction of $A$ to $\operatorname{span}\left(\operatorname{core}_{F_{\sigma}}(A)\right)$ is the same as the peripheral spectrum of the restriction of $A$ to $\operatorname{span} \tilde{\Phi}(v)$, counting algebraic multiplicities. Since $\widetilde{\Phi}(v)$ is a simplicial cone with $d_{\sigma}$ extreme rays and $A$ permutes these rays cyclically, it is not difficult to show (see, for instance, the proof of [Scha 3, Proposition 2]) that the eigenvalues of the restriction of $A$ to span $\widetilde{\Phi}(v)$ are simple, and are exactly $\rho_{F_{\sigma}}$ times all the $d_{\sigma}$ th root of unity. This completes the proof.

As an application of Theorem 3.14 we prove the following: 
Corollary 3.15. Let $K$ be a proper cone, and let $A \in \pi(K)$. Suppose that $\operatorname{core}(A)$ is a nonzero, simplicial cone. Let $\lambda$ be a positive number and let $k$ be a positive integer. If $x$ is an extremal distinguished eigenvector of $A^{k}$ corresponding to the nonzero distinguished eigenvalue $\lambda^{k}$, then $\sum_{i=0}^{k-1} \lambda^{-i} A^{i} x$ is an extremal distinguished eigenvector of $A$ corresponding to the eigenvalue $\lambda$.

Proof. Replacing $A$ by $A / \lambda$, we may assume that $\lambda=1$. Since $x$ is an extremal distinguished eigenvector of $A^{k}, \Phi(x)$ is a distinguished $A^{k}$-invariant face of $K$. By Theorem 3.14 there exists a unique cycle of $\tau_{A^{k}}$ that corresponds to $\Phi(x)$; say, the cycle is $\sigma$, of length $d$, and is composed of the ray $\operatorname{ray}(y)$ and its first $d-1$ iterates under the action of $A^{k}$. Then $\left(A^{k}\right)^{d} y=\alpha^{d} y$ for some $\alpha>0$; so the distinguished eigenvector of $A^{k}$ associated with the cycle $\sigma$ is for the eigenvalue $\alpha$. But by definition this eigenvector lies in relint $\Phi(x)$, and hence must be a positive multiple of $x$. It follows that $\alpha=1$, and replacing $x$ by a suitable positive multiple, we may assume that $x=\sum_{i=0}^{d-1} A^{i k} y$. Since $\operatorname{core}\left(A^{k}\right)=\operatorname{core}(A), \operatorname{ray}(y)$ is also an extreme ray of $\operatorname{core}(A)$; say, $\pi$ is the cycle of $\tau_{A}$ that contains $\operatorname{ray}(y)$ and is of length $l$. Then $A^{l} y=\beta^{l} y$ for some $\beta>0$. Since $y$ is a distinguished eigenvalue of $A^{d k}$ corresponding to the eigenvalue $1 \quad\left(=1^{d k}\right), \sum_{i=0}^{l-1} A^{i} y$ is the distinguished eigenvector of $A$ associated with the cycle $\pi$, and is for the eigenvalue 1 . (Refer to the last but one paragraph preceding Lemma 3.6). Repeating the argument that we have used at the beginning of our proof, we obtain $\beta=1$ and hence $A^{l} y=$ $y$. But $l$ is the smallest positive integer with this property, and as shown above $A^{d k} y=y$; hence $d k$ is divisible by $l$. So $\sum_{i=0}^{d k-1} A^{i} y$ is in fact just a positive multiple of $\sum_{i=0}^{l-1} A^{i} y$. Since $x=\sum_{i=0}^{d-1} A^{i k} y$, it is straightforward to verify that $\sum_{i=0}^{k-1} A^{i} x=\sum_{i=0}^{d k-1} A^{i} y$; thus $\sum_{i=0}^{k-1} A^{i} x$ is the distinguished eigenvector of $A$ associated with a cycle of $\tau_{A}$. So, by part (i) of Theorem $3.14 \sum_{i=0}^{k-1} A^{i} x$ generates a distinguished $A$-invariant face, and hence is an extremal distinguished eigenvector of $A$.

The example below shows that in Theorem 3.14 and Corollary 3.15 the hypothesis that $\operatorname{core}(A)$ be simplicial is indispensable.

Example 3.16. Let $K$ be the polyhedral cone in $\mathbb{R}^{3}$ with extreme vectors $y_{1}=(0,1,0)^{T}, y_{2}=(1,1,-1)^{T}, y_{3}=(1,0,0)^{T}$ and $y_{4}=(1,1,1)^{T}$. Then clearly there is (up to multiples) exactly one linear relation between these extreme vectors, namely, $2\left(y_{1}+y_{3}\right)=y_{2}+y_{4}$. Let $A$ be the $3 \times 3$ matrix determined uniquely by $A y_{1}=y_{3}, A y_{3}=y_{1}, A y_{2}=y_{4}$ and $A y_{4}=y_{2}$. (It is a well-defined linear mapping because it preserves the above linear relation.) Then $A \in \operatorname{Aut}(K)$, and so core $(A)=K$. Note that $K$ itself is the only nonzero $A$-invariant face, and hence also the only distinguished $A$-invariant face of $K$. But $\tau_{A}$ has two cycles. Also observe that the distinguished eigenvectors of $A$ associated with these cycles are the same, both being (multiples of) $y_{1}+y_{3}$.

Now take $B$ to be the $3 \times 3$ matrix $\operatorname{diag}(1,1,-1)$. It is readily checked that $B \in \operatorname{Aut}(K) ; B$ fixes $\operatorname{ray}\left(y_{1}\right)$ and $\operatorname{ray}\left(y_{3}\right)$, and exchanges $\operatorname{ray}\left(y_{2}\right)$ and $\operatorname{ray}\left(y_{4}\right)$. So $\tau_{B}$ has three cycles, but $K$ has only two distinguished $B$-invariant faces, namely, $\Phi\left(y_{1}\right)$ and $\Phi\left(y_{3}\right)$. The distinguished eigenvector of $B$ associated with the cycle formed by $\operatorname{ray}\left(y_{2}\right)$ and $\operatorname{ray}\left(y_{4}\right)$ lies in int $K$, and does not generate a distinguished $B$-invariant face. Also, it is clear that $y_{2}$ is an extremal 
distinguished eigenvector of $B^{2}$ corresponding to the eigenvalue 1. However, $y_{2}+B y_{2}$ is not an extremal distinguished eigenvector of $B$. (Cf. Corollary 3.15.)

We now give two further necessary conditions for $\operatorname{core}(A)$ to be polyhedral.

Theorem 3.17. Let $K$ be a proper cone, and let $A \in \pi(K)$. Suppose that $\operatorname{core}(A)$ is nonzero, polyhedral. Then there cannot exist two distinct extreme rays of $\operatorname{core}(A)$ both lying in the relative interior of the same face of $K$.

Proof. Assume to the contrary that $\operatorname{core}(A)$ has two distinct extreme rays, say, generated by $x$ and $y$, both lying in the relative interior of the same face of $K$, say $F$. Let $m$ be the order of the permutation $\tau_{A}$. Clearly $x, y$ are distinguished eigenvectors of $A^{m}$ both corresponding to the eigenvalue $\rho_{F}^{m}$. It follows that $x$ and $y$ both lie in the relative interior of the cone $\operatorname{span}\{x, y\} \cap K$, and this cone in turn is included in $\operatorname{core}(A)$. This contradicts the assumption that $x, y$ are extreme vectors of $\operatorname{core}(A)$.

Let $K$ be a proper cone, and let $A \in \pi(K)$. Following [Pul], we call an extreme vector $x$ of $\operatorname{core}(A)$ principal if (except for multiples) $x$ is the only extreme vector of core $(A)$ that lies in $\Phi(x)$; or in other words, $\Phi(x) \cap$ core $A$ is equal to $\operatorname{ray}(x)$.

Theorem 3.18. Let $K$ be a proper cone, and let $A \in \pi(K)$. Suppose that $\operatorname{core}(A)$ is polyhedral. If $x$ is a principal (nonprincipal) extreme vector of $\operatorname{core}(A)$, then so are $A^{i} x$ for $i=1,2, \ldots$.

Proof. First, let $x$ be a nonprincipal extreme vector of $\operatorname{core}(A)$. We are going to show that $A x$ (and hence also each $A^{i} x, i=1,2, \ldots$ ) is a nonprincipal extreme vector of $\operatorname{core}(A)$. Since $A$ maps $\operatorname{core}(A)$ onto itself, clearly $A x$ is an extreme vector of $\operatorname{core}(A)$. By definition of a nonprincipal extreme vector, there is an extreme vector $y$ of $\operatorname{core}(A)$, distinct from $x$, which lies in $\Phi(x)$. Then $A y$ is an extreme vector of core $(A)$ and lies in $\Phi(A x)$. As the restriction of $A$ to $\operatorname{span}(\operatorname{core}(A))$ is nonsingular, $A y$ must be distinct from $A x$. This proves that $A x$ is a nonprincipal extreme vector of $\operatorname{core}(A)$.

Now let $x$ be a principal extreme vector of $\operatorname{core}(A)$. Denote by $m$ the order of the permutation $\tau_{A}$. Then $A^{m} x$ is a positive multiple of $x$. If $A x$ is a nonprincipal extreme vector of $\operatorname{core}(A)$, then from what we have just proved, we infer that $x$ is also a nonprincipal extreme vector, which is a contradiction. This proves that each $A^{i} x, i=1,2, \ldots$, is a principal extreme vector of $\operatorname{core}(A)$.

\section{THE NONNEGATIVE MATRIX CASE}

We now derive our results on the core of a nonnegative matrix.

By the support of a vector $x=\left(\xi_{1}, \ldots, \xi_{n}\right)^{T}$ in $\mathbb{R}^{n}$ we mean the set $\{i \in$ $\left.\langle n\rangle: \xi_{i} \neq 0\right\}$. Let $P$ be a nonnegative matrix and let $\alpha$ be a class of $P$. We shall use $F_{\alpha}$ to denote the set of all vectors in $\mathbb{R}_{+}^{n}$ whose supports are included in the union of all classes having access to $\alpha$. For any vector $x \in \mathbb{R}_{+}^{n}$, it is readily checked that $\operatorname{supp}(P x)=\{i \in\langle n\rangle:(i, j)$ is an arc of $G(P)$ for some $j \in \operatorname{supp}(x)\}$. It follows that $F_{\alpha}$ is a $P$-invariant face of $\mathbb{R}_{+}^{n}$.

Lemma 4.1. Let $P$ be an $n \times n$ nonnilpotent nonnegative matrix. Then the association $\alpha \rightarrow F_{\alpha}$ is a one-to-one correspondence between the set of distin- 
guished classes of $P$ and the set of distinguished P-invariant faces of $\mathbb{R}_{+}^{n}$, both for nonzero distinguished eigenvalues.

Proof. To show that the association $\alpha \rightarrow F_{\alpha}$ is a well-defined mapping, let $\alpha$ be a distinguished class of $P$ for a nonzero distinguished eigenvalue $\lambda$. Then according to part (ii) of the Frobenius-Victory Theorem, there exists a distinguished eigenvector $x^{\alpha}$ of $P$ corresponding to $\lambda\left(=\rho_{F_{\alpha}}\right)$ that lies in relint $F_{\alpha}$. Furthermore, by part (iii) of the same theorem, the vector $x^{\alpha}$ is an extremal distinguished eigenvector. Hence $F_{\alpha}=\Phi\left(x^{\alpha}\right)$ and is a distinguished face of $\mathbb{R}_{+}^{n}$.

For different classes $\alpha_{1}, \alpha_{2}$ of $P$, it is clear that $F_{\alpha_{1}} \neq F_{\alpha_{2}}$, so the mapping $\alpha \rightarrow F_{\alpha}$ is one-to-one.

If $F$ is a distinguished face of $\mathbb{R}_{+}^{n}$ for a nonzero eigenvalue $\lambda$, then there exists an extremal distinguished eigenvector of $P$ that corresponds to $\lambda$ and generates $F$. But this vector must be equal to some $x^{\alpha}$ as given in part (iii) of the Frobenius-Victory Theorem, where $\alpha$ is a distinguished class such that $\rho\left(P_{\alpha \alpha}\right)=\lambda$. Hence $F=F_{\alpha}$, and the mapping $\alpha \rightarrow F_{\alpha}$ is onto. The proof is complete.

Recall that if $P$ is an $n \times n$ nonnegative matrix, then $D_{k}(P)$ (or simply $D_{k}$ ) denoted the cone generated by the distinguished eigenvectors of $P^{k}$ (in $\left.\mathbb{R}_{+}^{n}\right)$ corresponding to its nonzero distinguished eigenvalues.

Theorem 4.2. Let $P$ be an $n \times n$ nonnegative matrix with positive spectral radius. For each distinguished class $\alpha$ of $P$, denoted by $h_{\alpha}$ the index of imprimitivity of the irreducible submatrix $P_{\alpha \alpha}$, and also by $x^{\alpha}$ the extremal distinguished eigenvector of $P$ associated with the class $\alpha$ as given in part (ii) of the FrobeniusVictory Theorem. Then we have the following.

(i) For each cycle $\sigma$ of $\tau_{P}$ we associate with it the distinguished class $\alpha$ of $P$ with the property that $x^{\alpha}$ is the distinguished eigenvector of $P$ associated with the cycle $\sigma$. Then this association is a one-to-one correspondence between the set of cycles of $\tau_{P}$ and the set of distinguished classes of $P$ for nonzero distinguished eigenvalues. Furthermore, if $\sigma$ is a cycle of $\tau_{P}$ and $\alpha$ is the corresponding distinguished class, then the length of $\sigma$ is equal to $h_{\alpha}$.

(ii) $\operatorname{core}(P)$ is a simplicial cone with $\sum h_{\alpha}$ extreme rays, where the summation is taken over all distinguished classes $\alpha$ of $P$ for nonzero distinguished eigenvectors.

(iii) For any positive integer $k, \operatorname{core}(P)=D_{k}$ if and only if $k$ is a multiple of $q$, where $q$ is the least common multiple of all $h_{\alpha}$ with $\alpha$ running through all distinguished classes of $P$ for nonzero distinguished eigenvalues.

Proof. (i): First, by Corollary 3.3(ii) $\operatorname{core}(P)$ is a simplicial cone. Let $\sigma$ be a cycle of $\tau_{P}$. Then by Theorem 3.14 there exists a unique distinguished face of $\mathbb{R}_{+}^{n}$ for a nonzero distinguished eigenvalue with the property that the distinguished eigenvector of $P$ associated with the cycle $\sigma$ lies in its relative interior. By Lemma 4.1 this distinguished face can also be represented as $F_{\alpha}$ for a unique distinguished class $\alpha$ of $P$ for a nonzero distinguished eigenvalue. The vector $x^{\alpha}$ associated with the class $\alpha$ as described in part (ii) of the Frobenius-Victory Theorem lies in relint $F_{\alpha}$, and hence must be the distinguished eigenvector of $P$ associated with the cycle $\sigma$, as $F_{\alpha}$ is a distinguished face. This shows that the given association is a well-defined mapping from the set of cycles of $\tau_{P}$ to 
the set of distinguished classes of $P$ for nonzero distinguished eigenvalues. In fact, by Theorem 3.14 and Lemma 4.1 it is not difficult to see that this mapping is one-to-one and onto.

If $\sigma$ is a cycle and $\alpha$ is the corresponding distinguished class, then by Theorem 3.14(ii) the length of the cycle $\sigma$ is equal to the number of eigenvalues in the peripheral spectrum of $\left.P\right|_{\operatorname{span} F_{n}}$. But the latter quantity is also equal to $h_{\alpha}$, because $\left.P\right|_{\text {span } F_{\alpha}}$ can be represented by the submatrix of $P$ with row and column indices taken from classes having access to the distinguished class $\alpha$. So our second assertion follows.

(ii): It is clear that the number of extreme rays of the simplicial cone core $(P)$ is equal to the sum of lengths of the cycles of $\tau_{P}$. Hence, by the result of part (i) our assertion follows.

(iii): If $m$ denotes the order of permutation $\tau_{A}$, then it is clear that $m$ is equal to the least common multiple of the lengths of the cycles of $\tau_{P}$. So by Theorem 3.2 and the result of part (i) our result follows.

The following result is immediate.

Corollary 4.3. Let $P$ be a nonnegative matrix. Then $\operatorname{core}(P)$ is a single ray if and only if $P$ has exactly one initial class, this initial class is basic, and the corresponding submatrix of $P$ is primitive.

Corollary 4.4. Let $P$ be a nonnegative matrix. Then $\operatorname{core}(P)$ is a full cone if and only if each class of $P$ is a distinguished class for a nonzero distinguished eigenvalue, and the principal submatrix associated with each class of $P$ is a one-cycle matrix, that is, one which is permutationally similar to a matrix of the form

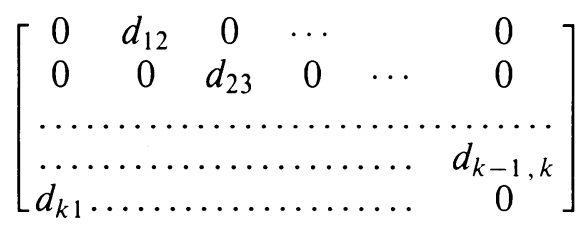

where $d_{12}, \ldots, d_{k 1}$ are all positive numbers.

Proof. By Theorem 4.2(ii) $\operatorname{core}(P)$ is always a simplicial cone. So, core $(P)$ is a full cone if and only if it has exactly $n$ extreme rays. Also by the same theorem core $(P)$ has $\sum h_{\alpha}$ extreme rays, where $h_{\alpha}$ denotes the index of imprimitivity of the submatrix $P_{\alpha \alpha}$ and the summation is taken over all the distinguished classes of $P$ for nonzero distinguished eigenvalues. It is also clear that $h_{\alpha}$ is always less than or equal to the order of $P_{\alpha \alpha}$ and that the equality holds if and only if $P_{\alpha \alpha}$ is a one-cycle matrix. So our theorem follows.

Berman and Plemmons [B-P, Chapter 1, Exercise 5.17] asked the question of when $\operatorname{core}_{K}(A)$ is a proper cone for a general proper cone $K$. In view of the above result and Examples 6.1, 6.2 in $\S 6$, we think that there is no simple answer to this question.

For a nonnegative matrix $P$, Theorem 4.2(i) tells us a connection between the cycles of the permutation $\tau_{P}$ and the distinguished classes of $P$. In what follows we shall give a more explicit description of the extreme rays of core $(P)$.

The following result is known (see [B-P, Chapter 2, Exercise 6.9]). We give an alternative proof from our viewpoint. 
Lemma 4.5. Let $P$ be an $n \times n$ irreducible nonnegative matrix with index of imprimitivity $h$. Let $q$ be a positive integer. Then $P^{q}$ is permutationally similar to a direct sum of $d$ irreducible matrices each having the same spectral radius where $d$ is the greatest common divisor of $q$ and $h$.

Proof. Without loss of generality, we may assume that $\rho(P)=1$. Since $P$ is irreducible, it has only one (basic) class. So by Theorem $4.2 \tau_{P}$ is a cycle of length $h$, say, consisted of the rays $\operatorname{ray}(x), \operatorname{ray}(P x), \ldots, \operatorname{ray}\left(P^{h-1} x\right)$. It is not difficult to show that then $\tau_{P}^{q}\left(=\tau_{P^{q}}\right)$ is a product of $d$ disjoint cycles each of length $q / d$, where $d$ is the greatest common divisor of $q$ and $h$. By Theorem 4.2(i) these $d$ cycles of $\tau_{P^{q}}$ give rise to $d$ distinct distinguished classes of $P^{q}$, and by Lemma 3.6 and the irreducibility of $P$ these classes are all for the eigenvalue 1 . Note that these $d$ distinguished basic classes of $P^{q}$ are all initial; otherwise, $P^{q}$ would have a distinguished class for an eigenvalue less than 1, and again by Lemma 3.6 we will arrive at a contradiction. As an irreducible nonnegative matrix $P$ has a unique eigenvector which lies in int $\mathbb{R}_{+}^{n}$; this eigenvector is clearly equal to the distinguished eigenvector associated with the cycle $\tau_{P}$ and hence, as can be readily verified, is a positive linear combination of the extremal distinguished eigenvectors of $P^{q}$ associated with the $d$ distinguished basic classes mentioned above. It follows that the union of these $d$ distinguished basic classes of $P^{q}$ is equal to $\langle n\rangle$. So our result follows.

Let $P$ be an $n \times n$ nonnegative matrix. Note that for any $i, j \in\langle n\rangle$, and any positive integer $q, i$ has access to $j$ in $G\left(P^{q}\right)$ if and only if there is a path from $i$ to $j$ in $G(P)$ with length a multiple of $q$. Hence if $i$, $j$ belong to the same class of $P^{q}$, then they also belong to the same class of $P$, but not conversely. It follows that every class of $P$ splits into (i.e. can be partitioned into) classes of $P^{q}$, and every class of $P^{q}$ is included in a unique class of $P$.

Corollary 4.6. Let $\alpha$ be a class of a nonnegative matrix $P$ such that $\rho\left(P_{\alpha \alpha}\right)>0$, and let $h_{\alpha}$ be the index of imprimitivity of $P_{\alpha \alpha}$. Then for any positive integer $q$, the class $\alpha$ of $P$ splits into $d_{\alpha}$ noncomparable classes of $P^{q}$, where $d_{\alpha}$ is the greatest common divisor of $q$ and $h_{\alpha}$. Furthermore, if $\alpha$ is a distinguished class, then the classes of $P^{q}$ into which it splits are also all distinguished.

Proof. From the preceding discussion, the class $\alpha$ of $P$ splits into classes of $P^{q}$. Note that for any $i, j \in \alpha$, every path of $G(P)$ from $i$ to $j$ (and vice versa) necessarily lies in $G\left(P_{\alpha \alpha}\right)$. So to determine how the class $\alpha$ splits, it suffices to consider the irreducible submatrix $P_{\alpha \alpha}$. Our assertion now follows from Lemma 4.5.

Last Part: Let $\alpha^{\prime}$ be a class of $P^{q}$ which is included in the class $\alpha$ of $P$. Let $\beta^{\prime}$ be a class of $P^{q}$ having access to $\alpha^{\prime}$ but not equal to $\alpha^{\prime}$. Let $\beta$ be the unique class of $P$ that includes $\beta^{\prime}$. Then clearly $\beta$ has access to $\alpha$ in $G(P)$. Furthermore, since $\alpha^{\prime}$ and $\beta^{\prime}$ are comparable classes of $P^{q}$, according to the first part of our result, the classes $\alpha$ and $\beta$ must be different. As $\alpha$ is a distinguished class of $P$, we have, $\rho\left(P_{\alpha \alpha}\right)>\rho\left(P_{\beta \beta}\right)$. Hence, we have, $\rho\left(\left(P^{q}\right)_{\alpha^{\prime} \alpha^{\prime}}\right)=\rho\left(P_{\alpha \alpha}\right)^{q}>\rho\left(P_{\beta \beta}\right)^{q}=\rho\left(\left(P^{q}\right)_{\beta^{\prime} \beta^{\prime}}\right)$. This proves that $\alpha^{\prime}$ is a distinguished class of $P^{q}$, as required.

Theorem 4.7. Let $P$ be a nonnegative matrix, and let $\alpha$ be a distinguished class of $P$ such that $\rho\left(P_{\alpha \alpha}\right)>0$. Let the index of imprimitivity of $P_{\alpha \alpha}$ be $h_{\alpha}$. Then $\operatorname{core}(P)$ has exactly $h_{\alpha}$ distinct (up to multiples) extreme vectors that lie in the 
$P$-invariant face $F_{\alpha}$ and whose supports meet $\alpha$. These $h_{\alpha}$ vectors generate the rays constituting the cycle of $\tau_{P}$ which corresponds to the class $\alpha$ in the sense of Theorem 4.2(i). Also, they are precisely the extremal distinguished eigenvectors of $P^{h_{\alpha}}$ associated with the distinguished classes of $P^{h_{\alpha}}$ into which the class $\alpha$ of $P$ splits.

Proof. Let $\sigma$ be the cycle of $\tau_{P}$ that corresponds to the distinguished class $\alpha$ (for a nonzero eigenvalue) in the sense of Theorem 4.2(i). By the same theorem, the length of $\sigma$ is equal to $h_{\alpha}$. Denote by $y$ the distinguished eigenvector of $P$ associated with the cycle $\sigma$. Since $y$ corresponds to the eigenvalue $\rho_{F_{\alpha}}$ of $P$ and lies in $F_{\alpha}$, clearly the $h_{\alpha}$ (distinct) vectors that generate the rays of the cycle $\sigma$ are extremal distinguished eigenvectors of $P^{h_{\alpha}}$ lying in $F_{\alpha}$ that correspond to the eigenvalue $\rho_{F_{\alpha}}^{h_{\alpha}}$. We claim that there is no other extremal distinguished eigenvector of $P^{h_{\alpha}}$ lying in $F_{\alpha}$ that corresponds to the eigenvalue $\rho_{F_{\alpha}}^{h_{\alpha}}$.

Suppose not. Let $x \in F_{\alpha}$ be another such extremal distinguished eigenvector. Then $\sum_{i=0}^{h_{\alpha}-1} \rho_{F_{\alpha}}^{-i} P^{i} x$ is a distinguished eigenvector of $P$ in $F_{\alpha}$ corresponding to $\rho_{F}$ and hence is a positive multiple of $y$, since $F_{\alpha}$ is a distinguished $P$-invariant face. As can be readily verified, since $x$ is an extremal distinguished eigenvector of $P^{h_{\alpha}}$ corresponding to $\rho_{F_{\alpha}}^{h_{\alpha}}$, so are the vectors $P^{i} x$, $i=1, \ldots, h_{\alpha}-1$. So we obtain two distinct representations of the vector $y$ in terms of the extremal distinguished eigenvectors of $P^{h_{\alpha}}$ : one in terms of the vectors that generate the rays of cycle $\sigma$, and the other in terms of the vectors $x, P x, \ldots, P^{h_{\alpha}-1} x$. This contradicts the fact that distinct extremal distinguished eigenvectors of a nonnegative matrix are linearly independent. This establishes our claim.

By Corollary 4.6 the class $\alpha$ of $P$ splits into $h_{\alpha}$ distinguished classes for the nonzero eigenvalue $\rho_{F_{\alpha}}^{h_{\alpha}}$, giving rise to $h_{\alpha}$ extremal distinguished eigenvectors of $P^{h_{\alpha}}$, which clearly belong to the face $F_{\alpha}$. Hence these $h_{\alpha}$ extremal distinguished eigenvectors of $P^{h_{\alpha}}$ are precisely the above-mentioned $h_{\alpha}$ extremal distinguished eigenvectors. Furthermore, since the support of each of these $h_{\alpha}$ vectors consists of all classes having access to one of the distinguished class into which $\alpha$ splits, it is clear that the supports of these vectors meet $\alpha$. Other extreme vectors of $\operatorname{core}(P)$ that lie in $F_{\alpha}$ belong to a cycle of $\tau_{P}$ different from $\sigma$, and the corresponding distinguished class of $P$ must be one which has access to $\alpha$, but different from $\alpha$. So the supports of the other extreme vectors of $\operatorname{core}(P)$ do not meet $\alpha$. The proof is complete.

If $P$ is a nonnegative matrix with positive spectral radius, then according to Theorem 4.2(ii) core $(P)$ has $\sum h_{\alpha}$ distinct extreme vectors, where $h_{\alpha}$ denotes the index of imprimitivity of the irreducible submatrix $P_{\alpha \alpha}$ and the summation is taken over all distinguished classes of $P$ for nonzero distinguished eigenvalues. By Theorem 4.7 a little bit more can be said: Each distinguished class $\alpha$ of $P$ for a nonzero eigenvalue gives rise to $h_{\alpha}$ distinct extreme vectors of $\operatorname{core}(P)$, which are precisely the extremal distinguished eigenvectors of $P^{h_{\alpha}}$ associated with the distinguished classes of $P^{h_{\alpha}}$ [as described in part (ii) of the Frobenius-Victory Theorem] into which the class $\alpha$ of $P$ splits.

Pullman [Pul, Lemma 6.1 and the preceding paragraph] has observed that for an irreducible nonnegative matrix $P, \tau_{P}$ is a single ray and the index of imprimitivity of $P$ is equal to the number of extreme rays of core $(P)$. Schaefer 
[Scha 3, Remark (i) following Proposition 1] also has made a parenthetic remark about the cycle of $\tau_{P}$ for a nonnegative matrix $P$ whose spectral radius is an eigenvalue of index one. Their observations clearly follow from our results.

\section{CONE-PRESERVING MAPS WITH SPECTRAL RADII OF INDEX ONE}

Pullman [Pul, Theorem 4] proved that if $A$ is a positive matrix, then $\operatorname{core}(A)$ is a ray generated by a positive vector. We shall give a generalization of this result. To do that, we need a number of lemmas. The first lemma follows from a standard compactness argument.

Lemma 5.1. Let $M$ be a compact metric space with metric $d$, and let $f$ be a continuous mapping of $M$ into itself. Let $\left(x_{i}\right)_{i \in \mathbb{N}}$ be a sequence in $M$ with $x_{i} \in f^{i}[M]$ for each $i$. Then $\lim _{i \rightarrow \infty} d\left(x_{i}, \bigcap_{k=1}^{\infty} f^{k}[M]\right)=0$, where $d\left(x_{i}, \bigcap_{k=1}^{\infty} f^{k}[M]\right)$ denotes the distance from $x_{i}$ to the set $\bigcap_{k=1}^{\infty} f^{i}[M]$.

Pullman [Pul, Lemma 2.2] gave the following result and its converse in the special case when $K$ is a polyhedral cone, but no proof was offered.

Lemma 5.2. Let $K$ be a proper cone, and let $A \in \pi(K)$. Also let $\|\cdot\|$ be a fixed norm of $\mathbb{R}^{n}$. If $\operatorname{core}(A)$ is a single ray generated by a unit vector $x$, then $A$ is nonnilpotent, and for every vector $y \in K \backslash \mathfrak{N}\left(A^{\nu(A)}\right)$, we have, $\lim _{i \rightarrow \infty} A^{i} y /\left\|A^{i} y\right\|=x$.

Proof. For convenience, denote $\nu(A)$ by $\nu$. It is clear that $A$ is nonnilpotent. Denote by $K_{1}$ the cone $\mathfrak{R}\left(A^{\nu}\right) \cap K$. Also denote by $\tilde{A}$ the mapping from $\mathfrak{R}\left(A^{\nu}\right) \cap S_{n-1}$ into itself given by $\tilde{A} x=A x /\|A x\|$, where $S_{n-1}$ is the unit sphere of $\mathbb{R}^{n}$ with respect to the norm $\|\cdot\|$. Let $y \in K \backslash \mathfrak{N}\left(A^{\nu}\right)$. Then $A^{\nu} y /\left\|A^{\nu} y\right\| \in$ $K_{1} \cap S_{n-1}$. It is easy to verify that for any vector $w, w \in \bigcap_{i \geqq 0} A^{i} K_{1}$ if and only if $w /\|w\| \in \bigcap_{i \geqq 0} \widetilde{A}^{i}\left(K_{1} \cap S_{n-1}\right)$. Since core $K_{K}(A)$ is just the single ray generated by $x$ and $\bigcap_{i \geqq 0} A^{i} K_{1}=\operatorname{core}_{K_{1}}(A)=\operatorname{core}_{K}(A)$, where the last equality has been shown in the proof of Theorem 2.2, it follows that we have $\bigcap_{i \geq 0} \widetilde{A}^{i}\left(K_{1} \cap S_{n-1}\right)=$ $\{x\}$. As $\tilde{A}$ is a continuous mapping of the metric space $K_{1} \cap S_{n-1}$ (with metric induced by the given norm of $\mathbb{R}^{n}$ ) into itself, we can now apply Lemma 5.1 with $M=K_{1} \cap S_{n-1}$ to conclude that $\lim _{i \rightarrow \infty} A^{i} y /\left\|A^{i} y\right\|=x$.

Lemma 5.3. Let $A \in \pi(K)$ where $K$ is a proper cone in $\mathbb{R}^{n}$. If $\rho(A)$ is a simple eigenvalue of $A$ with modulus greater than all other eigenvalues of $A$ and is also the only nonzero distinguished eigenvalue of $A$, then $\operatorname{core}(A)$ is a single ray.

Proof. We may assume that $\rho(A)=1$. Denote the cone $\mathfrak{R}\left(A^{\nu}\right) \cap K$ by $K_{1}$, and the restriction map $\left.A\right|_{\mathscr{R}\left(A^{\nu}\right)}$ by $B$, where $\nu=\nu(A)$. Then, as we have shown in the proof of Theorem 2.2, $\operatorname{core}_{K}(A)=\operatorname{core}_{K_{1}}(B)$. Note that any distinguished eigenvalue of $B$ for $K_{1}$ is necessarily a distinguished eigenvalue of $A$ for $K$. So by the given hypotheses on the eigenvalues of $A$, it follows readily that $\rho(B)$ is equal to 1 , is a simple eigenvalue of $B$, and is also the only distinguished eigenvalue of $B$ for $K_{1}$. (Since $B$ is nonsingular, 0 is not an eigenvalue of $B$.) So by [Tam 1, Theorem 5.4] $B^{T}$ (the adjoint of $B$ ) has an eigenvector, say $v$, in relint $K_{1}^{V}$ corresponding to 1 , where $K_{1}^{V}$ denotes the dual cone of $K_{1}$ in span $K_{1}$. Then, as can be readily shown, $C=\{y \in$ $\left.K_{1}:\langle y, v\rangle=1\right\}$ is a complete compact $B$-invariant cross-section of $K_{1}$. Let $u \in C$ be the (unique) eigenvector of $B$ corresponding to 1 . Clearly, every 
vector $x \in C$ can be written as $x=u+y$, where $y \in[\operatorname{span}\{v\}]^{\perp} \cap \operatorname{span} K_{1}$. Since 1 is a simple eigenvalue of $B$, (and $u \in C$ is already a corresponding eigenvector) it is clear that 1 cannot be an eigenvalue of the restriction of $B$ to $[\operatorname{span}\{v\}]^{\perp} \cap \operatorname{span} K_{1}$. The given hypothesis on $A$ also implies that except for $1, B$ has no other unimodular eigenvalues. Thus the spectral radius of the restriction of $B$ to $[\operatorname{span}\{v\}]^{\perp} \cap \operatorname{span} K_{1}$ is less than 1 and hence its $k$ th power tends to the zero operator as $k$ goes to infinity. Now it is not difficult to see that $\bigcap_{k=1}^{\infty} B^{k} C=\{u\}$. It follows that core $K_{1}(B)$, and hence core ${ }_{K}(A)$, is equal to the ray generated by the vector $u$.

For completeness, we also include the following result.

Theorem 5.4. Let $K$ be a proper cone, and let $A \in \pi(K)$. Denote by $\|\cdot\| a$ fixed norm of $\mathbb{R}^{n}$. If core con $_{K}(A)$ is a single ray, then $A$ is nonnilpotent and there exists a unit vector $x \in K$ such that $\lim _{i \rightarrow \infty} A^{i} y /\left\|A^{i} y\right\|=x$ for every vector $y \in K \backslash \mathfrak{N}\left(A^{\nu(A)}\right)$. The converse also holds, if either the index of $\rho(A)$ as an eigenvalue of $A$ is one or $\operatorname{core}_{K}(A)$ is polyhedral.

Proof. The firs part of our result follows from Lemma 5.2. Now we consider the converse parts. If $\nu_{\rho(A)}(A)=1$ then, using an argument similar to the one given in the proof of Lemma 5.3, we can show that $\operatorname{core}_{K}(A)$ is equal to $\operatorname{ray}(x)$. Next, suppose that $\operatorname{core}_{K}(A)$ is nonzero, polyhedral. Denote by $m$ the order of the permutation $\tau_{A}$. Then $A^{m}$ takes each extreme ray of $\operatorname{core}_{K}(A)$ onto itself, and by our hypothesis on the vector $x$, we have, for each nonzero extreme vector $y$ of $\operatorname{core}(A)$,

$$
x=\lim _{i \rightarrow \infty} A^{i} y /\left\|A^{i} y\right\|=\lim _{i \rightarrow \infty} A^{m i} y /\left\|A^{m i} y\right\|=y /\|y\| .
$$

It follows that $\operatorname{core}_{K}(A)$ is equal to $\operatorname{ray}(x)$.

Theorem 5.6. Let $K$ be a proper cone in $\mathbb{R}^{n}, n \geqq 2$, and let $A \in \pi(K)$. Then the following conditions are equivalent:

(a) $A$ is $K$-primitive.

(b) (i) $\mathfrak{N}(A) \cap K=\{0\}$, and (ii) core $(A)$ is a single ray generated by a vector in int $K$.

(c) $\rho(A)$ is the only distinguished eigenvalue of $A$ for $K$ and is a simple eigenvalue with modulus greater than all other eigenvalues of $A$, and the unique (up to multiples) distinguished eigenvector of $A$ lies in int $K$.

Proof. (b) $\Rightarrow(\mathrm{a})$ : Let $\operatorname{core}(A)$ be $\operatorname{ray}(u)$, where $u$ is a unit vector lying in int $K$. Since $\mathfrak{N}(A) \cap K=\{0\}$, for any nonzero vector $x \in K$, by Lemma 5.2, $\lim _{k \rightarrow \infty} A^{k} x /\left\|A^{k} x\right\|$ exists and is equal to $u$. Hence, for $k$ sufficiently large, we have, $A^{k} x \in \operatorname{int} K$. The $K$-primitivity of $A$ now follows from Barker [Bar 1, Proposition 2].

$(a) \Rightarrow(b)$ : Condition (b)(i) is clearly satisfied. Condition (b)(ii) follows from Lemma 5.3 and the fact that the unique eigenvector of a $K$-primitive matrix lies in int $K$.

(a) $\Rightarrow$ (c): Obvious.

(c) $\Rightarrow(\mathrm{b})$ : Note that 0 is not a distinguished eigenvalue of $A$. Otherwise, by condition (c) $A$ has an eigenvector in int $K$ corresponding to the eigenvalue 0 , and so $A$ is the zero matrix. As $n \geqq 2$, this contradicts the hypothesis that the distinguished eigenvector of $A$ is unique. So condition (b)(i) is satisfied. Condition (b)(ii) also follows readily from Lemma 5.3. 
We shall also give a necessary and sufficient condition for $A$ to be $K$ irreducible in terms of its core. But we need a lemma first.

Lemma 5.6. Let $K$ be a proper cone, and let $A$ be an $n \times n$ real matrix. Then $A \in \operatorname{Aut}(K)$ and $A$ has an eigenvector in int $K$ if and only if $A^{T} \in \operatorname{Aut}\left(K^{*}\right)$ and $A^{T}$ has an eigenvector in int $K^{*}$.

Proof. In general, for any $A \in \pi(K)$, we have, $A \in \operatorname{Aut}(K)$ if and only if $A^{T} \in \operatorname{Aut}\left(K^{*}\right)$. Here is the proof: Let $A^{T} \in \operatorname{Aut}\left(K^{*}\right)$. Then $A \in \pi(K)$ and $\left(A^{T}\right)^{-1} \in \pi\left(K^{*}\right)$. Hence $A^{-1}=\left(\left(A^{T}\right)^{-1}\right)^{T} \in \pi(K)$; so $A \in \operatorname{Aut}(K)$. By a duality argument we can also show that if $A \in \operatorname{Aut}(K)$ then $A^{T} \in \operatorname{Aut}\left(K^{*}\right)$.

To prove our theorem, it suffices to establish the "if" part; the "only if" part again follows by a duality argument. Now suppose that $A^{T} \in \operatorname{Aut}\left(K^{*}\right)$ and $A^{T}$ has an eigenvector $z$ in int $K^{*}$. As just proved, then $A \in \operatorname{Aut}(K)$. Assume that $A$ has no eigenvector in int $K$. Choose a distinguished eigenvector $x$ of $A$ corresponding to $\rho(A)$ such that $\operatorname{dim} \Phi(x)$ is maximal. Since $\Phi(x)$ is invariant under $A$, its dual face in $K^{*}$ (which consists of vectors in $K^{*}$ orthogonal to $\Phi(x))$ is invariant under $A^{T}$. Hence there exists a distinguished eigenvector $w$ of $A^{T}$ corresponding to, say the eigenvalue $\lambda$, such that $x$ and $w$ are orthogonal to each other. Since $z \in$ int $K^{*}$ and $-w \notin K^{*}$, replacing $w$ by a positive multiple, if necessary, we may assume that $z-w \in \partial K^{*}$. Then $A^{T}(z-w)=\rho(A)(z-(\lambda / \rho(A) w)) \in \partial K^{*}$, since $A^{T} \in \operatorname{Aut}\left(K^{*}\right)$ and $z-w \in \partial K^{*}$; hence $\lambda=\rho(A)$. So $z-w$ is also an eigenvector of $A^{T}$ lying in $\partial K^{*}$. Hence we can also find a distinguished eigenvector $y$ of $A$ (in $\partial K$ ) which is orthogonal to $z-w$. The assumption that $A^{T}$ has an eigenvector in int $K^{*}$ readily implies that the eigenvector $y$ of $A$ necessarily corresponds to the eigenvalue $\rho(A)$. Note that since $x$ is orthogonal to $w$ and $y$ is orthogonal to $z-w$, if $y \in \Phi(x)$ then $y$ is orthogonal to $z$, which cannot happen because $z \in$ int $K^{*}$. So $x+y$ is an eigenvector of $A$ corresponding to $\rho(A)$ such that $\operatorname{dim} \Phi(x+y)>\operatorname{dim} \Phi(x)$. This contradicts the maximality of $\operatorname{dim} \Phi(x)$.

Theorem 5.7. Let $K$ be a proper cone, and let $A \in \pi(K)$. Then $A$ is $K$ irreducible if and only if the following conditions are all satisfied:

(i) $\mathfrak{N}(A) \cap K=\{0\}$;

(ii) $\operatorname{core}(A) \cap \operatorname{int} K \neq \varnothing$; and

(iii) The restriction of $A$ to span core $(A)$ is irreducible with respect to $\operatorname{core}(A)$.

Proof. "Only if" part: It is clear that conditions (i) and (ii) are satisfied. Since $A$ is $K$-irreducible, $\rho(A)$ is a simple eigenvalue of $A$ and $A$ has (up to multiples) only one eigenvector lying in $K$ (in fact in int $K$ ), say $x$. Also this vector is the only eigenvector of $A$ lying in $\operatorname{core}(A)$. Thus $\left.A\right|_{\text {span } \operatorname{core}(A)}$ has only one distinguished eigenvalue for $\operatorname{core}(A)$ and the index of its spectral radius is one. So the adjoint of $\left.A\right|_{\text {span core }(A)}$ has an eigenvector lying in the relative interior of the dual cone of $\operatorname{core}(A)$ in its own linear span; hence, by Lemma 5.6, $\left.A\right|_{\text {span core }(A)}$ has an eigenvector lying in the relative interior of $\operatorname{core}(A)$. Thus the only eigenvector of $A$ in $\operatorname{core}(A)$ lies in its relative interior. This establishes condition (iii).

"If" part: Assume that conditions (i) and (ii) are satisfied. We contend that if $A$ is $K$-reducible, then the restriction of $A$ to $\operatorname{span} \operatorname{core}(A)$ is reducible with respect to core $(A)$. Let $x$ be an eigenvector of $A$ in $\partial K$. By condition (i), $x$ must correspond to a nonzero distinguished eigenvector of $A$; hence, 
$x \in \operatorname{core}(A)$. Choose a vector $y \in \operatorname{core}(A) \cap \operatorname{int} K$. Then $x-\alpha y$ does not lie in $K$, and hence also not in $\operatorname{core}(A)$ for any $\alpha>0$. It follows that $x$ is an eigenvalue of $A$ lying in $\operatorname{rbd}[\operatorname{core}(A)]$. So condition (iii) is not satisfied.

In passing, we note that in Theorem 5.7 if we replace the word "irreducible" by "primitive" (in two places) the result still holds. The corresponding result is, in fact, just a restatement of Theorem $5.5(\mathrm{a}) \Leftrightarrow(\mathrm{b})$. The point is, if $A \in \operatorname{Aut}(K)$ and is $K$-primitive, then necessarily $K$ is a one-dimensional cone.

Corollary 5.8. Let $K$ be a proper cone, and let $A \in \pi(K)$. Suppose that $\operatorname{core}(A)$ is a nonzero polyhedral cone. Then $A$ is $K$-irreducible if the following conditions are all satisfied:

(i) $\mathfrak{N}(A) \cap K=\{0\}$;

(ii) $\operatorname{core}(A) \cap \operatorname{int} K \neq \varnothing$; and

(iii) $\tau_{A}$ is a single ray.

If, in addition, $\operatorname{core}(A)$ is simplicial, then the converse also holds.

Proof. In view of Theorem 5.7, it suffices to show that $\left.A\right|_{\mathrm{span} \operatorname{core}(A)}$ is irreducible with respect to core $(A)$. Assume that the contrary holds. Then $A$ has an eigenvector, say $x$, lying in $\operatorname{rbd}[\operatorname{core}(A)]$. By Lemma $3.7 A$ maps the face of core $(A)$ generated by $x$ onto itself, and hence $A$ permutes the extreme rays of this face among themselves. This contradicts the hypothesis that $\tau_{A}$ is a single cycle.

Last Part: Suppose that $A$ is $K$-irreducible and that $\operatorname{core}(A)$ is a simplicial cone. If $\tau_{A}$ is not a single cycle, then the distinguished eigenvector of $A$ associated with any cycle of $\tau_{A}$ lies in $\operatorname{rbd}[\operatorname{core}(A)]$, and hence by Theorem 5.7 $A$ is reducible, which is a contradiction. So condition (iii) (and also conditions (i) and (ii)) must be satisfied.

At the end of his paper, Pullman [Pul] also gave geometric characterization of irreducibility for a nonnegative matrix. His characterization contains condition (i) and (iii) of Corollary 5.8 (with $K$ replaced by $\mathbb{R}_{+}^{n}$ ) and with condition (ii) replaced by the following stronger condition:

(ii) $^{\prime}: \mathbb{R}^{n}$ can be written as a direct sum of $d$ coordinate subspaces each containing exactly one extreme ray of $\operatorname{core}(A)$ in its positive orthant.

The reason why condition (ii) ${ }^{\prime}$ is always satisfied by an $n \times n$ irreducible nonnegative matrix $A$ can now be readily seen as follows. Denote by $h$ the index of imprimitivity of $A$. Then by Corollary 4.6 the unique class of $A$ splits into $h$ noncomparable distinguished classes of $A^{h}$, and these $h$ classes are precisely all the classes of $A^{h}$. But by Theorem 4.2(i) and the discussion after Theorem $4.7 \tau_{A}$ is a single cycle of length $h$, whose rays are generated by extremal distinguished eigenvectors of $A^{h}$ associated with the distinguished classes into which the unique class of $A$ splits. So it is clear that condition (ii) follows.

In [Scha 3, Proposition 1] Schaefer gave several equivalent conditions on a nonnegative matrix for its spectral radius to be an eigenvalue of index one. We shall extend this result to the setting of a linear mapping preserving a proper cone. But we need the following result, which has interest of its own.

Theorem 5.9. Let $A$ be an $n \times n$ real matrix. The following conditions on $A$ are equivalent: 
(a) $A$ is nonzero, diagonalizable (over $\mathbb{C}$ ), all eigenvalues of $A$ are of the same moduli and $\rho(A)$ is an eigenvalue of $A$.

(b) There exists a proper cone $K$ such that $A \in \operatorname{Aut}(K)$ and $A$ has an eigenvector in int $K$.

(c) There exists a proper cone $K$ such that $A \in \pi(K)$, and for any such cone $K$, we have, $A \in \operatorname{Aut}(K)$.

(d) There exists a proper cone $K$ such that $A \in \pi(K)$, and for any such cone $K$, we have, $A \in \operatorname{Aut}(K)$ and $A$ has an eigenvector in int $K$.

(e) $A$ is nonzero, and there exists a proper cone $K$ such that $A / \rho(A)$ takes a complete cross-section of $K$ onto itself.

Proof. (b) $\Rightarrow(\mathrm{a})$ : Clearly $\rho(A) \in \sigma(A)$ and $\rho(A) \neq 0$. Since $A$ has an eigenvector in int $K, \nu_{\rho(A)}(A)=1$ and $\rho(A)$ is the only distinguished eigenvalue of $A^{T}$ (for $K^{*}$ ). By the Perron-Schaefer condition, every eigenvalue of $A$ of modulus $\rho(A)$ is also of index one. It remains to show that all eigenvalues of $A$ are of the same moduli. As $A \in \operatorname{Aut}(K)$, we also have $A^{T} \in \operatorname{Aut}\left(K^{*}\right)$, and hence $\left(A^{T}\right)^{-1} \in \pi\left(K^{*}\right)$. By the Perron-Frobenius theorem $\rho\left(\left(A^{T}\right)^{-1}\right)$ is a distinguished eigenvalue of $\left(A^{T}\right)^{-1}$ (for $\left.K^{*}\right)$, and hence $\left(\rho\left(\left(A^{T}\right)^{-1}\right)\right)^{-1}$ is a distinguished eigenvalue of $A^{T}$. Since $A^{T}$ has only one distinguished eigenvalue, necessarily, we have $\rho(A)=\left(\rho\left(\left(A^{T}\right)^{-1}\right)\right)^{-1}$. But $\left(\rho\left(\left(A^{T}\right)^{-1}\right)\right)^{-1}$ is also an eigenvalue of $A^{T}$ of the least modulus, it follows that all eigenvalues of $A^{T}$, and hence of $A$, are of the same moduli.

(a) $\Rightarrow(\mathrm{c})$ : Since $A$ satisfies the Perron-Schaefer condition, there exists a proper cone $K$ such that $A \in \pi(K)$. Consider one such proper cone $K$. By Theorem 3.9(i) (and in its notation), there exists a sequence $\left((A / \rho(A))^{q_{i}}\right)_{i \in \mathbb{N}}$ of positive powers of $A / \rho(A)$ converging to $P$, which in this case is the identity matrix. It follows that $A$ is nonsingular, and $A^{-1} \quad\left(=\lim _{i \rightarrow \infty} A^{q_{i}-1} /(\rho(A))^{q_{i}}\right) \in$ $\pi(K)$. This proves that $A \in \operatorname{Aut}(K)$.

(c) $\Rightarrow(\mathrm{a})$ : By the Perron-Schaefer condition, certainly $\rho(A)$ is an eigenvalue of $A$. Suppose that $A$ is not diagonalizable, or $A$ has an eigenvalue with modulus less than $\rho(A)$. Then for the proper cone $K$ obtained by Vandergraft's construction (see [Van, Theorem 3.1] or [B-P, Theorem 1.3.2]) one can check that $A \in \pi(K)$, but $A \notin \operatorname{Aut}(K)$.

(c) $\Rightarrow(\mathrm{d})$ : Let $K$ be a proper cone such that $A \in \pi(K)$. Then by condition (c) $A \in \operatorname{Aut}(K)$. From what we have just proved, condition (a) is also satisfied. Hence $\rho(A)$ is the only distinguished eigenvalue of $A$ and is of index one. So $A^{T}$ has an eigenvector in int $K^{*}$, and in view of Lemma 5.6, it follows that $A$ also has an eigenvector in int $K$.

(d) $\Rightarrow($ b): Obvious.

(b) $\Rightarrow(\mathrm{e})$ : By condition (b) and Lemma 5.6 there exists a proper cone $K$ such that $A \in \operatorname{Aut}(K)$ and $A^{T}$ has an eigenvector, say $u$, in int $K^{*}$ (corresponding to $\rho(A))$. It is straightforward to verify that $\{x \in K:\langle x, u\rangle=1\}$ is a complete cross-section of $K$, and that $A / \rho(A)$ takes this cross-section onto itself.

$(\mathrm{e}) \Rightarrow(\mathrm{b})$ : Suppose that there exists a proper cone $K$ and a complete crosssection $C$ of $K$ such that $(A / \rho(A)) C=C$. Then clearly $A \in \operatorname{Aut}(K)$. Note that the hypersubspace $W=$ aff $C-$ aff $C$ meets the cone $K$ only at the zero vector. By the Gordan-Stiemke theorem (see, for instance, [B-T-D, Corollary 2.6]), $W^{\perp} \cap$ int $K^{*} \neq \varnothing$. Since $C$ is invariant under $A / \rho(A)$, so is $W$; hence $W^{\perp}$ is also invariant under $A^{T}$. But the latter subspace is one-dimensional, so 
$A^{T}$ has an eigenvector in int $K^{*}$, and by Lemma 5.6 again, $A$ has an eigenvector in int $K$.

In passing, we note that Theorem 5.9 implies the observation made by Horne [Hor, Theorem 3.6 and the subsequent comment] on an irreducible automorphism of a cone. Also, using [S-W, Theorem 3.6], we can show that in condition (b) of Theorem 5.9 we can replace the words "a proper cone" by "an ellipsoidal cone" (that is one which is linearly isomorphic to an ice-cream cone).

We need a new notation and a new definition in order to state our result. For a given norm $\tau$ of $\mathbb{R}^{n}$, we denote by $\|\cdot\|_{\tau}$ the corresponding induced matrix norm. Given a proper cone $K$ of $\mathbb{R}^{n}$, a norm $\tau$ on $\mathbb{R}^{n}$ is said to be strictly $K$-monotone on a linear subspace $M$ of $\mathbb{R}^{n}$ if $x, y \in M$ and $0^{K} \leqq x^{K} \leqq y$, $x \neq y$, implies $\tau(x)<\tau(y)$.

Theorem 5.10. Let $K$ be a proper cone of $\mathbb{R}^{n}$, and let $A \in \pi(K)$ be such that $\rho(A)>0$. Denote by $M$ the direct sum of the (real) generalized eigenspaces of A corresponding to eigenvalues in its peripheral spectrum. Consider the following conditions:

(a) $A$ is an automorphism of the cone $M \cap K$ when restricted to its linear span. $M$.

(b) $\|A\|_{\tau}=\tau(A)$ for some norm $\tau$ of $\mathbb{R}^{n}$ which is strictly $K$-monotone on

(c) $\|A\|_{\tau}=\rho(A)$ for some norm $\tau$ of $\mathbb{R}^{n}$.

(d) The set of all positive powers of $A / \rho(A)$ is bounded in the euclidean metric of $\mathbb{R}^{n, n}$.

(e) Each eigenvalue in the peripheral spectrum of $A$ is of index one.

(f) $\rho(A)$ is an eigenvalue of $A$ of index one.

Then conditions (b), (c), (d), (e) and (f) are all equivalent. Condition (a) is always implied by conditions (b)-(f), and is equivalent to them when $K$ is polyhedral.

Proof. Replacing $A$ by $A / \rho(A)$, henceforth, we assume that $\rho(A)=1$.

For any complex matrix $A$, it is known that conditions (c), (d) and (e) are equivalent: the equivalence of (d) and (e) follows from a consideration of the asymptotic behaviour of the powers of the Jordan form of $A$; that (c) and (e) are equivalent follows from [Dok, Theorem 5], where two more equivalent conditions are also given. (See also [Els 1, Theorem 3.2].) In the present case when $A$ is a cone-preserving map, by the Perron-Schaefer condition, (f) is another equivalent condition. That $(\mathrm{b}) \Rightarrow$ (c) holds is obvious.

(e) $\Rightarrow(b)$ : We first consider the special case when each eigenvalue of $A$ is unimodular. By Theorem 5.9 and Lemma 5.6 $A \in \operatorname{Aut}(K)$ and $A^{T}$ has an eigenvector in int $K^{*}$. By a similarity transformation, we may assume that $A$ is in real canonical form with an identity matrix put in the left top corner. We may also assume that $e_{1} \in \operatorname{int} K^{*}$. Then $C=\left\{x \in K:\left\langle x, e_{1}\right\rangle=1\right\}$ is a complete, compact cross-section of $K$. Choose $a>0$ sufficiently large, so that $C$ is included in the compact convex set $\left\{\left(1, \xi_{2}, \ldots, \xi_{n}\right)^{T} \in \mathbb{R}^{n}: a \geqq\left(\sum_{i=2}^{n} \xi_{i}^{2}\right)^{1 / 2}\right\}$. Let $\widehat{K}$ be the proper cone of $\mathbb{R}^{n}$ generated by $C$. Define a norm $\tau$ on $\mathbb{R}^{n}$ by $\tau\left(\left(\xi_{1}, \ldots, \xi_{n}\right)^{T}\right)=\left(a^{2} \xi_{1}^{2}+\sum_{i=2}^{n} \xi_{i}^{2}\right)^{1 / 2}$. It is straightforward to check that the norm $\tau$ is strictly $\widehat{K}$-monotone (on $\mathbb{R}^{n}$ ), and hence strictly $K$-monotone, as $\widehat{K} \supseteq K$. Furthermore, $A$ is an isometry with respect to $\tau$, and hence we have $\|A\|_{\tau}=1$. 
Now consider the general case. We may assume that $A$ is of the form $A_{1} \oplus$ $A_{2}$, where each eigenvalue of $A_{1}$ is unimodular and $\rho\left(A_{2}\right)<1$. Denote the coordinate subspace of $\mathbb{R}^{n}$ corresponding to the blocks $A_{i}$ of $A$ by $W_{i}, i=$ 1,2. Then $M=W_{1}$, and (noting that by Theorem $3.9 M=\operatorname{span}(M \cap K)$ ) from the first part of our proof there exists a norm $\tau_{1}$ of $W_{1}$ which is strictly $(M \cap K)$-monotone such that $\|A\|_{\tau_{1}}=1$. Since $\rho\left(A_{1}\right)<1$, there also exists a norm $\tau_{2}$ of $W_{2}$ such that $\left\|A_{2}\right\|_{\tau_{2}}<1$ (see, for instance, [Dok, Lemma 4]). For any vector $x=x_{1}+x_{2} \in \mathbb{R}^{n}$, with $x_{1} \in W_{1}$ and $x_{2} \in W_{2}$, define $\tau(x)$ to be $\left[\tau_{1}\left(x_{1}\right)^{2}+\tau_{2}\left(x_{2}\right)^{2}\right]^{1 / 2}$. It is clear that the norm $\tau$ satisfies condition (b).

(e) $\Rightarrow$ (a): It is clear that $A(M \cap K) \subseteq M \cap K$. Since condition (e) is satisfied, by Theorem $5.9 A$ is an automorphism of $M \cap K$ when restricted to its linear span.

Now we establish $(\mathrm{a}) \Rightarrow(\mathrm{f})$ under the additional hypothesis that $K$ is polyhedral. Assume to the contrary that $1 \quad(=\rho(A))$ is an eigenvalue of $A$ of index $\geqq 2$. Since $K$ is polyhedral, by [Tam 1, Theorem 7.5], the generalized eigenspace of $A$ corresponding to 1 has a basis consisted of vectors in $K$. So, in particular, $K$ contains a generalized eigenvector of $A$ corresponding to 1 that is of order $\geqq 2$. Clearly, this vector belongs to $M \cap K$. Now $M \cap K$ is a polyhedral cone and by condition (a) $A$ is an automorphism of $M \cap K$ when restricted to its linear span; so according to Corollary $3.5 \mathrm{~A}$ cannot have a generalized eigenvector of order $\geqq 2$ lying in $M \cap K$. Thus we arrive at a contradiction.

It is illuminating to compare our Theorem 3.9(ii) and Theorem 5.10(a) with [Gro, Proposition 2.1(a)]. Also note that the lemma of [V-L] follows readily from our results.

By Theorem 5.10 it is clear that when $K$ is a polyhedral cone and $A \in$ $\pi(K)$ the conditions (a)-(f) as given there are equivalent. In his paper [Scha 3, Proposition 1] Schaefer also gave the same set of equivalent conditions for a nonnegative matrix except that $\mathbb{R}^{n}$ is replaced by $\mathbb{C}^{n}$ (which we could also have done) and that condition (a) is replaced by the following:

$(a)^{\prime} M$ has a basis, consisting of nonnegative vectors, on which $A / \rho(A)$ acts as a permutation.

We now explain why in the case of a nonnegative matrix $A$ condition $(a)^{\prime}$ is also another equivalent condition. First, suppose that the equivalent conditions of our theorem are satisfied. Denote by $d$ the order of the permutation induced by $A$ on the extreme rays of $M \cap \mathbb{R}_{+}^{n}$. Then from the last part of the proof the above theorem, $\left.A^{d}\right|_{\operatorname{span}\left(M \cap \mathbb{R}_{+}^{n}\right)}$ is the identity map (assuming again $\rho(A)=1$ for simplicity). But since $\nu_{1}(A)=1$, by Theorem 3.9(ii) $\operatorname{span}(M \cap K)=M$; thus $M \subseteq \mathfrak{N}\left(I-A^{d}\right)$. A consideration of the $d$ th power of the Jordan form of $A$ shows that if $x$ is an eigenvector of $A^{d}$ corresponding to 1 , then $x$ belongs to the direct sum of eigenspaces of $A$ corresponding to eigenvalues which are $d$ th roots of unity. Hence we have $\mathfrak{N}\left(I-A^{d}\right) \subseteq M$ and so the equality holds. By the Frobenius-Victory Theorem, $\mathfrak{N}\left(I-A^{d}\right) \cap \mathbb{R}_{+}^{n}$ is a simplicial cone, and by condition (a) $A$ permutes the extreme rays of this cone. But $\left.A^{d}\right|_{M}$ is the identity map, so we can choose a basis for $M$ which consists of extreme vectors of this simplicial cone in such a way that $A$ acts as a permutation on the basis vectors. 
Now suppose that condition $(a)^{\prime}$ is satisfied. Let $d$ be the order of the permutation $A / \rho(A)$ acts on the said basis of $M$. Then $\left.(A / \rho(A))^{d}\right|_{M}$ is the identity map. Hence, $M$ cannot contain a generalized eigenvector of $A$ corresponding to $\rho(A)$ of order $\geqq 2$. It follows that condition ( $\mathrm{f}$ ) is satisfied.

A final remark about Theorem 5.10 is in order. We know that condition (a) does not imply the equivalent conditions (b)-(f), even when $\operatorname{core}(A)$ or $M \cap K$ is a simplicial cone. As a counterexample, consider the cone $K$ and the matrix $A$ given in [T-W, Example 3.7]. It is not difficult to show that in this case $\operatorname{core}(A)$ and $M \cap K$ are both equal to the single ray generated by the eigenvector $(1,0,0)^{T}$. So condition (a) is satisfied. However, the index of $\rho(A) \quad(=1)$ as an eigenvalue of $A$ is two.

\section{EXAMPLES AND A FURTHER RESULT}

Example 6.1. Consider the ice-cream cone $K=\left\{\left(\xi_{1}, \xi_{2}, \xi_{3}\right)^{T} \in \mathbb{R}^{n}: 2 \xi_{1} \xi_{3} \geqq\right.$ $\left.\xi_{2}^{2}, \xi_{1}, \xi_{3} \geqq 0\right\}$, whose axis of rotation is generated by the vector $(1,0,1)^{T}$. The cross-section of $K$ by the hyperplane $\xi_{3}=1$ is the convex set $C$ bounded by the parabola $2 \xi_{1}=\xi_{2}^{2}, \xi_{3}=1$. Note that this cross-section meets every ray of $K$ except the one generated by the vector $(1,0,0)^{T}$. Let $A$ be the $3 \times 3$ matrix

$$
A=\left[\begin{array}{ccc}
1 & 1 & 1 / 2 \\
0 & 1 & 1 \\
0 & 0 & 1
\end{array}\right] .
$$

By direct calculations, we have,

$$
A\left(\xi^{2} / 2, \xi, 1\right)^{T}=\left((\xi+1)^{2} / 2, \xi+1,1\right)^{T} \text { for any real number } \xi .
$$

Hence $A$ maps the above-mentioned parabola onto itself. Since $C$ is the convex hull of this parabola, it follows that $A$ maps $C$ onto itself, and hence $A \in$ $\operatorname{Aut}(K)$. (The ray generated by $(1,0,0)^{T}$ is fixed by $A$.) In other words, $\operatorname{core}_{K}(A)$ is $K$ itself. On the other hand, one can check directly, or by the Rothblum Index Theorem (see [Rot, Theorem 3.1(2)]), that $A$ is similar to an elementary Jordan matrix of order three corresponding to the eigenvalue 1 . It follows that for any nonzero vector $y \in K$, we have, $\lim _{i \rightarrow \infty} A^{i} y /\left\|A^{i} y\right\|=$ $(1,0,0)^{T}$. So, without additional assumptions, the converse part of Theorem 5.4 does not hold. Clearly $K$ contains generalized eigenvectors of $A$ of order $\geqq 2$. So Corollary 3.4 is not valid if the polyhedrality assumption on $\operatorname{core}(A)$ is dropped.

Now consider the proper cone $\widetilde{K}=\left\{\left(\xi_{1}, \xi_{2}, \xi_{3}\right)^{T} \in \mathbb{R}_{+}^{3}: 2 \xi_{1} \xi_{3} \geqq \xi_{2}^{2}\right\}$, i.e. half of an ice-cream cone. Let $\widetilde{C}$ denote the cross-section of $\widetilde{K}$ by the hyperplane $\xi_{3}=1$. Take the same matrix $A$ as above. One can readily show that $\widetilde{A} C \subseteq \widetilde{C}$ and that $\bigcap_{i=1}^{\infty} A^{i} \widetilde{C}$ is the empty set. It follows that $A \in \pi(\widetilde{K})$ and core $_{\widetilde{K}}(A)$ is the single ray generated by the vector $(1,0,0)^{T}$. (Note that $\operatorname{core}_{\mathbb{R}_{+}^{3}}(A)$ is also a single ray, according to Corollary 4.3.) So even for the same cone-preserving map, for different cones, the corresponding cores can be quite different in nature.

Example 6.2. Let $\alpha$ be a real number such that $0<\alpha<1 / 2$. Let $\widetilde{C}$ be the convex set in $\mathbb{R}^{2}$ with extreme points $\left(k, \alpha^{k-1}\right)^{T}, k=0, \pm 1, \pm 2, \ldots$. Then the recession cone of $\widetilde{C}$ is given by

$$
O^{+} \widetilde{C}=\operatorname{pos}\left\{(1,0)^{T},(0,1)^{T}\right\} \text {. }
$$


Let $C_{0}$ be the unbounded closed convex set in $\mathbb{R}^{2}$ given by:

$$
\begin{aligned}
C_{0}=\left\{x \in \mathbb{R}^{2}: x=x_{1}+x_{2} \text { for some } x_{1}\right. \\
\left.\quad \in \operatorname{conv}\left\{(0,0)^{T},\left(k, \alpha^{k-1}\right)^{T}, k=-1,-2, \ldots\right\} \text { and } x_{2} \in O^{+} \widetilde{C}\right\} .
\end{aligned}
$$

Then as can be readily seen, $(0,0)^{T}$ and $\left(k, \alpha^{k-1}\right)^{T}, k=-1,-2, \ldots$, are precisely the extreme points of $C_{0}$ and also $C_{0}$ and $\widetilde{C}$ have the same recession cone. Let $\widetilde{K}$ be the proper cone of $\mathbb{R}^{3}$ that arises from $\widetilde{C}$ in the standard way; that is,

$$
\widetilde{K}=\left\{\lambda\left(\begin{array}{l}
1 \\
x
\end{array}\right): x \in \widetilde{C}, \lambda \geqq 0\right\} \cup \operatorname{pos}\left\{(0,1,0)^{T},(0,0,1)^{T}\right\} .
$$

Also let $K$ be the proper cone of $\mathbb{R}^{3}$ that arises from $C_{0}$ in the standard way. Let

$$
A=\left[\begin{array}{lll}
1 & 0 & 0 \\
1 & 1 & 0 \\
0 & 0 & \alpha
\end{array}\right] .
$$

By direct calculations, we can show that $A K$ is the proper cone of $\mathbb{R}^{3}$ that arises from $C_{1}$ in the standard way, where $C_{1}$ is the closed convex set in $\mathbb{R}^{2}$ with extreme points $(1,0)^{T},\left(k, \alpha^{k-1}\right)^{T}, k=0,-1,-2, \ldots$, and with the same recession cone as that of $C_{0}$. It is easily seen that $C_{1} \subseteq C_{0}$, so $A \in \pi(K)$. More generally, for $i=1,2, \ldots, A^{i} K$ is the proper cone that arises from $C_{i}$ in the standard way, where $C_{i}$ is the closed convex set in $\mathbb{R}^{2}$ with extreme points $(i, 0)^{T},\left(k, \alpha^{k-1}\right)^{T}, k=i-1, i-2, \ldots$, and again with the same recession cone as that of $C_{0}$. It is not difficult to see that $\bigcap_{k=0}^{\infty} C_{k}=\widetilde{C}$. Hence, we have, $\operatorname{core}_{k}(a)=\widetilde{K}$. Note that in this example $\operatorname{core}_{K}(A)$ has infinitely many extreme rays in int $K$. So the conclusion of Theorem 3.17 does not hold here. Note also that $\alpha$ is a nonzero distinguished eigenvalue of $A$ for $K$, different from 1, but $\operatorname{core}_{K}(A)$ is an indecomposable cone. So in Corollary 3.3(i) the polyhedrality assumption on $\operatorname{core}(A)$ is also indispensable.

Let $K$ be a proper cone of $\mathbb{R}^{n}$, and let $A \in \pi(K)$. By Remark 3.10 it is clear that if $y$ is a nonzero vector in $K$ such that for some $0 \neq x \in K$, some subsequence of $\left(A^{k} x /\left\|A^{k} x\right\|\right)_{k \in \mathbb{N}}$ converges to $y /\|y\|$ (where $\|\cdot\|$ is some norm of $\mathbb{R}^{n}$ ), then $y$ belongs to core ${ }_{K}(A)$. A careful examination of the proof given by Birkhoff [Bir] of the Perron-Schaefer condition for a cone-preserving map will reveal that the set of all such vectors $y$ is, in fact, included in $\bigcup_{\lambda}\left(W_{\lambda} \cap K\right)$ where the union is taken over all nonzero distinguished eigenvalues $\lambda$ of $A$ and $W_{\lambda}$ denotes the direct sum of all (real) eigenspaces of $A$ corresponding to eigenvalues with modulus equal to $\lambda$, our next result will strengthen this observation.

Theorem 6.3. Let $K$ be a proper cone in $\mathbb{R}^{n}$, and let $A \in \pi(K)$. For each nonzero distinguished eigenvalue $\lambda$ of $A$, denote by $W_{\lambda}$ the direct sum of all (real) eigenspaces of $A$ corresponding to eigenvalues with modulus equal to $\lambda$. For each positive integer $k$, let $D_{k}$ have the same meaning as in Lemma 3.1. Then $\bigcup_{i=1}^{\infty} D_{i} \subseteq \bigoplus\left(W_{\lambda} \cap K\right) \subseteq \operatorname{core}(A)$, where the direct sum is taken over all nonzero distinguished eigenvalues $\lambda$ of $A$. When $\operatorname{core}(A)$ is polyhedral, the inclusions become equalities. 
Proof. For each nonzero distinguished eigenvalue $\lambda$ of $A$, clearly, $A\left(W_{\lambda} \cap K\right) \subseteq$ $W_{\lambda} \cap K$. So by Theorem $5.9 A$ takes $W_{\lambda} \cap K$ onto itself; hence, we have, $\bigoplus\left(W_{\lambda} \cap K\right) \subseteq \operatorname{core}(A)$. Let $\mu$ be a nonzero distinguished eigenvalue of $A^{i}$, where $i$ is a positive integer. Then by Lemma 3.6 if $\lambda$ is the positive $i$ th root of $\mu$, then $\lambda$ is a distinguished eigenvalue of $A$. But by considering the $i$ th power of the Jordan form of $A$, we obtain $\mathfrak{N}\left(\mu I-A^{i}\right) \subseteq W_{\lambda}$. It follows that we have the inclusion $\bigcup_{i=1}^{\infty} D_{i} \subseteq \bigoplus\left(W_{\lambda} \cap K\right)$, where the direct sum is taken over all nonzero distinguished eigenvalues $\lambda$ of $A$. If $K$ is polyhedral, then by Theorem 3.2 and Lemma 3.1(iv), we have, $\operatorname{core}(A)=\bigcup_{i=1}^{\infty} D_{i}$; hence the inclusions become equalities.

In general, the inclusions in the above theorem cannot be replaced by equalities. Choose $K$ to be the 3-dimensional ice-cream cone, and let $A$ be a rotation about its axis through an angle which is not a rational multiple of $\pi$. Then $\bigcup_{k=1}^{\infty} D_{k} \neq \oplus\left(W_{\lambda} \cap K\right)$. Example 6.1 shows that the inclusion $\bigoplus\left(W_{\lambda} \cap K\right) \subseteq \operatorname{core}(A)$ can be strict. Also note that for each positive integer $k$, the set for $A^{k}$ corresponding to $\bigoplus\left(W_{\lambda} \cap K\right)$ is the same as that for $A$; this follows from Lemma 3.6 (and [L-T, Theorem 9.4.7]). So one does not obtain a set included in $\operatorname{core}(A)$ which is larger than $\bigoplus\left(W_{\lambda} \cap K\right)$ by taking into account all the positive powers of $A$.

In view of Examples 6.1, 6.2 and Theorem 6.3 one might wonder whether core $_{K}(A)$ is always included in the direct sum of the (real) generalized eigenspaces of $A$ corresponding to eigenvalues with modulus equal to some nonzero distinguished eigenvalues. It is not hard to see that this is false. As a counterexample, take $K$ to be the 3 -dimensional ice-cream cone $\left\{\left(\xi_{1}, \xi_{2}, \xi_{3}\right)^{T}\right.$ : $\left.\left(\xi_{1}^{2}+\xi_{2}^{2}\right)^{1 / 2} \leqq \xi_{3}\right\}$ and take $A$ to be the matrix that satisfies

$$
2 A=\left[\begin{array}{ccc}
4 & \sqrt{ } 3 & \sqrt{ } 5 \\
0 & \sqrt{ } 5 & 1 \\
2 \sqrt{ } 3 & 2 & 2 \sqrt{ } 5
\end{array}\right] .
$$

(This example is borrowed from [Hor, Example 5.5].) One can check that in this case $A \in \operatorname{Aut}(K)$, and that $A$ has distinct positive eigenvalues $\lambda_{1}, 2, \lambda_{2}$, arranged in decreasing order. Furthermore, the eigenvalues $\lambda_{1}$ and $\lambda_{2}$ are both distinguished eigenvalues of $A$ for $K$, but not the eigenvalue 2. So it is clear that the above-mentioned conjecture is wrong.

A major problem in concern with the core of a cone-preserving map certainly is, to describe vectors in core $_{K}(A)$ in terms of $A$ and $K$. Also, we are interested in finding equivalent conditions for $\operatorname{core}_{K}(A)$ to be polyhedral or simplicial, because as we have shown in $\S 3$ many interesting results about cone-preserving maps hold under one of these conditions as a basic assumption. However, in view of the example given in this section, these problems seem intractable in the general case. In order to have any further fruitful investigations, we modify our problems slightly as follow: Given an $n \times n$ real matrix $A$ that satisfies the Perron-Schaefer condition, find an equivalent condition on $A$ for which there exists a proper cone $K$ such that $A \in \pi(K)$ and $\operatorname{core}_{K}(A)$ is polyhedral or simplicial. We shall solve these problems completely in the next section.

\section{Matrices with the Perron-Schaefer condition}

Let $A$ be an $n \times n$ complex matrix. For any eigenvalue $\lambda$ of $A$, let $E_{\lambda}^{(0)}$ denote the projection of $\mathbb{C}^{n}$ onto $\mathfrak{N}\left((\lambda I-A)^{\nu_{\lambda}}\right)$ [the generalized eigenspace of 
$A$ corresponding to $\lambda$ ] along $\mathfrak{R}\left((\lambda I-A)^{\nu_{\lambda}}\right)$ [the direct sum of the generalized eigenspaces of $A$ corresponding to eigenvalues other than $\lambda$ ], where we use $\nu_{\lambda}$ to denote $\nu_{\lambda}(A)$ for simplicity. Also define the components of $A$ by

$$
E_{\lambda}^{(r)}=(A-\lambda I)^{r} E_{\lambda}^{(0)}, \quad r=0,1, \ldots
$$

It is known that if $K$ is a proper cone of $\mathbb{R}^{n}$ and $A \in \pi(K)$ then $E_{\rho(A)}^{(\nu-1)} \mid \mathbb{R}^{n}$ is nonzero and belongs to $\pi(K)$, where $\nu=\nu_{\rho(A)}$ (see, for instance, [Schn 1, Theorem 5.2]).

We shall need the following result, which has interest of its own. We use $J_{k}(\lambda)$ to denote the $k \times k$ upper triangular elementary Jordan matrix that corresponds to the eigenvalue $\lambda$.

Theorem 7.1. Let $K$ be a proper cone of $\mathbb{R}^{n}$, and let $A \in \pi(K)$ with $\rho(A)>0$. Denote $\nu_{\rho(A)}(A)$ by $\nu$. Then we have the following:

(i) There is a subsequence of $\left((\nu-1) ! A^{k} /\left[\rho(A)^{k-\nu+1} k^{\nu-1}\right]\right)_{k \in \mathbb{N}}$ which converges to $\sum_{\lambda} E_{\lambda}^{(\nu-1)}$, where the summation runs through all eigenvalues $\lambda$ in the peripheral spectrum of $A$ with the same index as that of $\rho(A)$. Hence $\left.\left(\sum_{\lambda} E_{\lambda}^{(\nu-1)}\right)\right|_{\mathbb{R}^{n}} \in \pi(K)$.

(ii) Let $M$ denote the intersection of $\mathbb{R}^{n}$ with $\bigoplus_{\lambda}\left[(\lambda I-A)^{\nu-1} \mathfrak{N}\left((\lambda I-A)^{\nu}\right)\right]$, where $\lambda$ runs through the same set of eigenvalues as that described in the sum appearing in part (i). Then $M=\operatorname{span}(M \cap K)$ and $M \cap K \subseteq \operatorname{core}(A)$.

Proof. (i): Let $P$ be a nonsingular matrix such that $P^{-1} A P$ is a Jordan form of $A$, which we denote by $J$. Suppose that $J=\bigoplus_{j=1}^{r} J_{m_{j}}\left(\lambda_{j}\right)$. Rearranging the blocks of $J$, if necessary, we may assume that the blocks which correspond to eigenvalues in the peripheral spectrum of $A$ and are of order $\nu$ are among the first $h(\leqq r)$ blocks. For large values of $k$, the dominant entries of $J^{k}$ occur within the first $h$ blocks and are exactly at their right upper corners. For the $j$ th block, $1 \leqq j \leqq h$, this entry is $\left(\begin{array}{c}k \\ \nu-1\end{array}\right) \lambda_{j}^{k-\nu+1}$, which is of the same order of approximation as

$$
\left[k^{\nu-1} \rho(A)^{k-\nu+1} e^{i(k-\nu+1) \theta_{j}}\right] /(\nu-1) !,
$$

where $\theta_{j}$ satisfies $\lambda_{j}=\rho(A) e^{i \theta_{j}}$. By letting $k$ goes to $+\infty$ through a suitable increasing sequence of natural numbers, we can obtain $e^{i(k-\nu+1) \theta_{j}} \rightarrow 1$ for $j=1, \ldots, h$. (Refer to the proof of Theorem 3.9(i).) Hence, if we multiply $A^{k}$ by $(\nu-1) ! /\left(k^{\nu-1} \rho(A)^{k-\nu+1}\right)$, and letting $k$ go to $\infty$ through the abovementioned sequence of natural numbers, our sequence of matrices will tend to $\sum_{j=1}^{h} E_{\lambda_{j}}^{(\nu-1)}$ (in the space of $n \times n$ complex matrices). Because $K$ is a closed cone, it is clear that $\left.\left(\sum_{j=1}^{h} E_{\lambda_{j}}^{(\nu-1)}\right)\right|_{\mathbb{R}^{n}} \in \pi(K)$.

(ii): As is well known the range space of $\left.\left(\sum_{\lambda} E_{\lambda}^{(\nu-1)}\right)\right|_{\mathbb{R}^{n}}$ is equal to $M$, where $M$ is as given, and the summation is taken over all $\lambda$ as described in the statement of (i). Since $K$ is a full cone of $\mathbb{R}^{n},\left(\sum_{\lambda} E_{\lambda}^{(\nu-1)}\right) K$ is a full cone of $M$. But $\left(\sum_{\lambda} E_{\lambda}^{(\nu-1)}\right) K \subseteq M \cap K$, it follows that we have $\operatorname{span}(M \cap K)=M$. By Theorem 5.9 it is clear that $A(M \cap K)=M \cap K$. So we also have $M \cap K \subseteq$ $\operatorname{core}(A)$.

We note, in passing, that the subspace $M$ considered in Theorem 7.1(ii) can also be described as the direct sum of all subspaces of the form 


$$
\begin{aligned}
&(\lambda I-A)^{\nu-1} \mathfrak{N}\left((\lambda I-A)^{\nu}\right) \text { or } \\
& {\left[\left(a^{2}+b^{2}\right) I-2 a A+A^{2}\right]^{\nu-1} \mathfrak{N}\left(\left[\left(a^{2}+b^{2}\right) I-2 a A+A^{2}\right]^{\nu}\right), }
\end{aligned}
$$

where $\lambda, a+i b(a, b \in \mathbb{R}, b \neq 0)$ are respectively real and nonreal eigenvalues in the peripheral spectrum of $A$ with the same index as that of $\rho(A)$.

The following result extends our previous Corollary 3.11.

Corollary 7.2. Let $K$ be a proper cone of $\mathbb{R}^{n}$, and let $A \in \pi(K)$. If $\operatorname{core}(A)$ is polyhedral, then every eigenvalue in the peripheral spectrum of $A$ with the same index as that of $\rho(A)$ is equal to $\rho(A)$ times a root of unity.

Proof. Let $\mu$ be an eigenvalue in the peripheral spectrum of $A$ with the same index as that of $\rho(A)$. Let $M$ denote the subspace

$$
\mathbb{R}^{n} \cap \bigoplus\left[(\lambda I-A)^{(\nu-1)} \mathfrak{N}\left((\lambda I-A)^{\nu}\right)\right],
$$

where the direct sum is taken over all eigenvalues $\lambda$ in the peripheral spectrum of $A$ with the same index as that of $\rho(A)$. Then $\mu$ is in the peripheral spectrum of $\left.A\right|_{M}$, and hence also in that of $\left.A\right|_{\operatorname{span}(\operatorname{core}(A))}$, in view of Theorem 7.1(ii). But $\operatorname{core}(A)$ is polyhedral, so by [B-T, Theorem 2] $\mu$ must be equal to $\rho(A)$ times a root of unity.

We shall show that the condition given in Corollary 7.2 is, in fact, an equivalent condition for the existence of a proper cone $K$ invariant under $A$ (which satisfies the Perron-Schaefer condition) such that $\operatorname{core}_{K}(A)$ is polyhedral. To establish the reversed direction, we need a sequence of lemmas.

Lemma 7.3. Let $A$ be an $n \times n$ real matrix. Let $K_{1}$ and $K_{2}$ be closed, pointed cones of $\mathbb{R}^{n}$ both invariant under $A$.

(i) If $\operatorname{core}_{K_{1}}(A) \cap\left(-\operatorname{core}_{K_{2}}(A)\right)=\{0\}$ and $\operatorname{span} K_{1} \cap \operatorname{span} K_{2} \cap \mathfrak{N}\left(A^{\nu(A)}\right)=\{0\}$, then $K_{1} \cap\left(-K_{2}\right)=\{0\}$.

(ii) If $K_{1} \cap\left(-K_{2}\right)=\{0\}$ then $K_{1}+K_{2}$ is a closed, pointed cone, and we have

$$
\text { core }_{K_{1}+K_{2}}(A)=\operatorname{core}_{K_{1}}(A)+\operatorname{core}_{K_{2}}(A) .
$$

Proof. (i): Assume to the contrary that there exists a nonzero vector $x \in K_{1} \cap$ $\left(-K_{2}\right)$. Let $u$ be the limit of some convergent subsequence of the sequence $\left(A^{i} x /\left\|A^{i} x\right\|\right)_{i \in \mathbb{N}}$. (Note that by our hypothesis $A^{i} x \neq 0$ for all $i$.) Then using Remark 3.10, we infer that the nonzero vector $u$ belongs to core $K_{K_{1}}(A) \cap$ $\left(-\right.$ core $\left._{K_{2}}(A)\right)$, which contradicts our hypothesis.

(ii): It is straightforward to verify the pointedness of the cone $K_{1}+K_{2}$; by [Roc, Corollary 9.1.2] its closedness also follows.

The inclusion core $K_{1}+K_{2}(A) \supseteq \operatorname{core}_{K_{1}}(A)+\operatorname{core}_{K_{2}}(A)$ is obvious. To prove the reversed inclusion, let $z \in \operatorname{core}_{K_{1}+K_{2}}(A)$. Then for each positive integer $i$, there exist vectors $x_{i}^{(1)} \in A^{i} K_{1}$ and $x_{i}^{(2)} \in A^{i} K_{2}$ such that $z=x_{i}^{(1)}+x_{i}^{(2)}$. By a compactness argument, we can readily show that the sequences $\left(x^{(1)}\right)_{i \in \mathbb{N}}$ and $\left(x_{i}^{(2)}\right)_{i \in \mathbb{N}}$ are both bounded. Let $\left(x_{k_{i}}^{(1)}\right)_{i \in \mathbb{N}}$ and $\left(x_{k_{i}}^{(2)}\right)_{i \in \mathbb{N}}$ be convergent subsequences with limits $y_{1}$ and $y_{2}$ respectively. Then $z=y_{1}+y_{2}$. But by Remark 3.10, we have, $y_{i} \in \operatorname{core}_{K_{i}}(A), i=1,2$; so the desired reversed inclusion follows.

Lemma 7.4. Let $K_{1}$ and $K_{2}$ be proper cones in possibly different euclidean spaces. Let $A_{1} \in \pi\left(K_{1}\right)$ and $A_{2} \in \pi\left(K_{2}\right)$, each with spectral radius equal to 
one. Suppose that for each $\lambda \in \sigma\left(A_{1}\right) \cap \sigma\left(A_{2}\right)$, the geometric multiplicities of $\lambda$ as an eigenvalue of $A_{1}$ or of $A_{2}$ are both equal to one. Also assume that $\nu_{1}\left(\left.A_{2}\right|_{\text {span core }\left(A_{2}\right)}\right)=1$. Then there exists a rank-one matrix $y z^{T}$ such that the matrix

$$
\left[\begin{array}{cc}
A_{1} & y z^{T} \\
0 & A_{2}
\end{array}\right]
$$

belongs to $\pi\left(K_{1} \times K_{2}\right)$, its core relative to $K_{1} \times K_{2}$ is core $_{K_{1}}\left(A_{1}\right) \times\{0\}$, and the geometric multiplicity of each $\lambda$ in $\sigma\left(A_{1}\right) \cap \sigma\left(A_{2}\right)$ as an eigenvalue of this matrix is equal to one.

Proof. Suppose that the given cones $K_{1}$ and $K_{2}$ lie in the euclidean spaces $\mathbb{R}^{n_{1}}$ and $\mathbb{R}^{n_{2}}$ respectively. Choose a vector $y$ from int $K_{1}$ that does not belong to $\bigcup_{\mu}\left[\mathfrak{R}\left(A_{1}-\mu I\right) \cap \mathbb{R}^{n_{1}}\right]$, where the union is taken over $\mu$ in $\sigma\left(A_{1}\right) \cap \sigma\left(A_{2}\right)$, and the range spaces are taken over $\mathbb{C}^{n_{1}}$. Also choose a vector $z$ from int $K_{2}^{*}$ that does not belong to the union of the orthogonal complements (in $\mathbb{R}^{n_{2}}$ ) of eigenspaces of $A_{2}$ corresponding to real eigenvalues, or of real eigenspaces of $A_{2}$ corresponding to conjugate pairs of nonreal complex eigenvalues in $\sigma\left(A_{1}\right) \cap$ $\sigma\left(A_{2}\right)$. Now, let $A$ be the matrix

$$
\left[\begin{array}{cc}
A_{1} & y z^{T} \\
0 & A_{2}
\end{array}\right]
$$

We are going to show that this matrix possesses the desired properties. It is straightforward to check that $A \in \pi\left(K_{1} \times K_{2}\right)$. Let $\lambda \in \sigma\left(A_{1}\right) \cap \sigma\left(A_{2}\right)$, and let $\left[\begin{array}{l}u \\ v\end{array}\right]$, where $u \in \mathbb{C}^{n_{1}}$ and $v \in \mathbb{C}^{n_{2}}$, be a corresponding eigenvector of $A$. We contend that $v=0$. Once this is proved, we would obtain $u \in \mathfrak{N}\left(A_{1}-\lambda I\right)$; but the nullity of $A_{1}-\lambda I$ is one, hence so is $A-\lambda I$. By direct calculations, we have $\left(A_{1}-\lambda I\right) u+\left(z^{T} v\right) y=0$ and $\left(A_{2}-\lambda I\right) v=0$. By our choice of $y$, clearly $z^{T} v=0$. If $v$ is nonzero, then the real vector $z$ will be orthogonal to the 1-dimensional subspace $\mathfrak{N}\left(A_{2}-\lambda I\right)$ of $\mathbb{C}^{n}$, and hence to $\mathfrak{N}\left(A_{2}-\lambda I\right) \cap \mathbb{R}^{n_{2}}$ if $\lambda$ is real, or to the (2-dimensional) real eigenspace corresponding to the conjugate pair $\lambda, \bar{\lambda}$ if $\lambda$ is nonreal, in contrary to our choice of $z$. This proves our contention.

Now, we are going to prove that $\operatorname{core}_{K_{1} \times K_{2}}(A)=\operatorname{core}_{K_{1}}\left(A_{1}\right) \times\{0\}$. It is clear that $\operatorname{core}(A) \supseteq \operatorname{core}\left(A_{1}\right) \times\{0\}$. Let $\left[\begin{array}{l}u \\ v\end{array}\right] \in \operatorname{core}(A)$, where $u \in \mathbb{R}^{n_{1}}$ and $v \in \mathbb{R}^{n_{2}}$. Since $A$ maps $\operatorname{core}(A)$ onto itself, for each positive integer $k$, there exists a unique vector $\left[\begin{array}{l}x_{k} \\ y_{k}\end{array}\right] \in \operatorname{core}(A)$ such that $A^{k}\left[\begin{array}{l}x_{k} \\ y_{k}\end{array}\right]=\left[\begin{array}{l}u \\ v\end{array}\right]$. Then $A_{2}^{k} y_{k}=v$ for each positive integer $k$; hence, $v$ belongs to core $\left(A_{2}\right)$, and so does $y_{k}$ for each $k$. Choose an eigenvector $x$ of $A_{1}$ in $K_{1}$ corresponding to the eigenvalue 1 $\left(=\rho\left(A_{1}\right)\right)$ such that $y \geqq{ }^{K_{1}} x$. (This is possible, because $y \in \operatorname{int} K_{1}$.) By direct calculations, we have,

$$
\begin{aligned}
u & =A_{1}^{k} x_{k}+\left(\sum_{i=0}^{k-1} A_{1}^{i}\left(y z^{T}\right) A_{2}^{k-1-i}\right) y_{k} \\
& \geqq^{K_{1}} \sum_{i=0}^{k-1}\left\langle z,\left(\left.A_{2}\right|_{\text {span } \operatorname{cor}\left(A_{2}\right)}\right)^{-(i+1)} v\right\rangle A_{1}^{i} x \\
& =\sum_{i=0}^{k-1}\left\langle z, y_{i+1}\right\rangle x
\end{aligned}
$$


Since $1\left(=\rho\left(A_{2}\right)\right)$ is an eigenvalue of $\left.A_{2}\right|_{\text {span core }\left(A_{2}\right)}$ of index one, by [Dok, Theorem 5] there exists some norm $\tau$ of span core $\left(A_{2}\right) \operatorname{such}$ that $\left\|\left.A_{2}\right|_{\operatorname{span} \operatorname{core}\left(A_{2}\right)}\right\|_{\tau}=$ 1 , where $\|\cdot\|_{\tau}$ is the corresponding induced matrix norm. So, for each positive integer $i, \tau(v) \leqq\left\|\left.A_{2}\right|_{\text {span } \operatorname{core}\left(A_{2}\right)}\right\|_{\tau}^{i} \tau\left(y_{i}\right)=\tau\left(y_{i}\right)$. Let $\zeta=\min \{\langle z, w\rangle: w \in$ core $\left._{K_{2}}\left(A_{2}\right), \tau(w)=\tau(v)\right\}$. Then, for each positive integer $k$, we have, $u \geq K_{1}$ $\sum_{i=0}^{k-1} \zeta x=k \zeta x$. For this to happen, necessarily, $\zeta=0$. But $z \in$ int $K_{2}^{*}$, it follows that $v=0$. Now it is also clear that $u \in \operatorname{core}\left(A_{1}\right)$. This shows that $\operatorname{core}(A) \subseteq \operatorname{core}\left(A_{1}\right) \times\{0\}$, and hence the equality holds. The proof is complete.

For convenience, we shall use $e_{i}^{(n)}$ to denote the vector of $\mathbb{R}^{n}$ with 1 at its $i$ th entry and 0 elsewhere.

Lemma 7.5. For any positive integer $s$ and any $r \times r$ real matrix $B$ with $\rho(B)<$ 1 (where $B$ may be an empty matrix), there exists a proper polyhedral cone $K$ of $\mathbb{R}^{r+s}$ such that $J_{s}(1) \oplus B \in \pi(K)$ and $\operatorname{core}_{K}\left(J_{s}(1) \oplus B\right)$ is a single ray generated by the unique distinguished eigenvector of $J_{S}(1) \oplus B$ corresponding to the eigenvalue 1.

Proof. If $B$ is an empty matrix, we choose $K$ to be $\mathbb{R}_{+}^{s}$. Then clearly $J_{s}(1) \in$ $\pi(K)$ and by Corollary $4.3 \operatorname{core}_{K}\left(J_{S}(1)\right)$ is a single ray. So, henceforth, we assume that $r$ is a positive integer. We first deal with the case when $s=1$. Let $C=\operatorname{conv}\left\{ \pm B^{j} e_{i}^{(r)}: 1 \leqq i \leqq r, j=0,1,2, \ldots\right\}$. As $\rho(B)<1$, we have $\lim _{j \rightarrow \infty} B^{j}=0$. So, for $j$ sufficiently large, the points $\pm B^{j} e_{i}^{(r)}, 1 \leqq i \leqq r$, all belong to the interior of the convex set $\operatorname{conv}\left\{ \pm e_{i}^{(r)}: 1 \leqq i \leq r\right\}$. It follows that $C$ is a symmetric polytope, with nonempty interior, and is invariant under $B$; in fact, $\bigcap_{j=0}^{\infty} B^{j} C=\{0\}$. Let $K_{1}$ be the closed cone of $\mathbb{R}^{r+1}$ that arises from $C$ in the standard way. Then it is clear that $K_{1}$ is a proper polyhedral cone, $J_{1}(1) \oplus B \in \pi\left(K_{1}\right)$ and $\operatorname{core}_{K_{1}}\left(J_{1}(1) \oplus B\right)=\operatorname{ray}\left(e_{1}^{r+1}\right)$. Furthermore, $e_{1}^{(r+1)}$ is the unique distinguished eigenvector of $J_{1}(1) \oplus B$ (corresponding to 1) in $K_{1}$.

Now consider the case when $s>1$. Denote by $W$ the coordinate subspace of $\mathbb{R}^{r+s}$ corresponding to the block $B$ of $J_{s}(1) \oplus B$. From the first part of our proof, there exists a proper polyhedral cone $K_{1}$ of $\operatorname{span}\left\{e_{1}^{(r+s)}\right\} \oplus W$ invariant under $J_{s}(1) \oplus B$ such that $\operatorname{core}_{K_{1}}\left(J_{s}(1) \oplus B\right)=\operatorname{ray}\left(e_{1}^{(r+s)}\right)$. Let $K_{2}$ be the nonnegative orthant of the coordinate subspace corresponding to the first block of $J_{s}(1) \oplus B$. Then $K_{2}$ is invariant under $J_{s}(1) \oplus B$, and by Corollary 4.3 core $_{K_{2}}\left(J_{2}(1) \oplus B\right)=\operatorname{ray}\left(e_{1}^{(r+s)}\right)$. Now take $K=K_{1}+K_{2}$. Clearly $K$ is invariant under $A$. Furthermore, by Lemma 7.3 it is not difficult to see that $K$ is a proper cone of $\mathbb{R}^{r+s}$ and also that $\operatorname{core}_{K}\left(J_{s}(1) \oplus B\right)$ is equal to $\operatorname{ray}\left(e_{1}^{(r+s)}\right)$.

For any real number $\theta$, let $R_{\theta}$ denote the $2 \times 2$ rotation matrix

$$
\left[\begin{array}{cc}
\cos \theta & \sin \theta \\
-\sin \theta & \cos \theta
\end{array}\right]
$$

If $m$ is a positive integer and $A$ is a $k \times k$ matrix, we use $J_{m}(A)$ to denote 
the upper block triangular matrix

$$
\left[\begin{array}{llllll}
A & I & & & & \\
& A & (\bullet) & & & \\
& & & (\bullet) & & \\
& & & & (\bullet) & I \\
& & & & & A
\end{array}\right]
$$

with $m$ blocks $A$ on the main diagonal, $m-1 k \times k$ identity matrices on the superdiagonal, and zero elsewhere.

Lemma 7.6. Let $\theta$ be a real number which is not an integral multiple of $\pi$. For any positive integers $r, s$ with $s \geqq r$, there exists a proper cone $K$ of $\mathbb{R}^{2 r+s}$ such that $J_{s}(1) \oplus J_{r}\left(R_{\theta}\right) \in \pi(K)$ and $\operatorname{core}_{K}\left(J_{s}(1) \oplus J_{r}\left(R_{\theta}\right)\right)$ is equal to $\operatorname{ray}\left(e_{1}^{(2 r+s)}\right)$ if $s>r$, or a 3-dimensional cone that contains $e_{1}^{(3 r)}$ in its relative interior if $s=r$. In case $s=r$, if $C$ is any proper cone of the direct sum of the (real) eigenspaces of $J_{r}(1) \otimes J_{r}\left(R_{\theta}\right)$ that contains $e_{1}^{(3 r)}$ in its relative interior, then the cone $K$ can also be constructed in such a way that $\operatorname{core}_{K}\left(J_{r}(1) \oplus J_{r}\left(R_{\theta}\right)\right) \subseteq C$. If, in addition, $\theta$ is a rational multiple of $\pi$, then $K$ can always be chosen to be polyhedral.

Proof. We first treat the case when $s=r$. It is clear that the (euclidean) unit disc $D$ of $\mathbb{R}^{2}$ is mapped onto itself under the matrix $R_{\theta}$. Let $K_{1}$ be the closed, pointed cone that arises from $D$ in the standard way. Then $K_{1}$ is a proper cone of $\mathbb{R}^{3}$ and $J_{1}(1) \oplus J_{1}\left(R_{\theta}\right) \quad\left[=(1) \oplus R_{\theta}\right] \in \operatorname{Aut}\left(K_{1}\right)$; hence our assertion holds for $r=1$. For $r>1$, using Lemma 7.4 repeatedly, we can find an upper block triangular matrix $T$ with $r$ block equal to (1) $\oplus R_{\theta}$ along its main diagonal, which leaves invariant the cone $K_{1} \times \cdots \times K_{1}$ ( $r$ times) and is such that its core relative to this cone is equal to $K_{1} \times\{0\} \times \cdots \times\{0\} \quad(\{0\}$, occurring $r-1$ times). Furthermore, by the same lemma, $T$ is similar to $J_{r}(1) \oplus J_{r}\left(R_{\theta}\right)$. It is clear that the corrdinate subspace of $\mathbb{R}^{3 r}$ corresponding to the first block of $T$ is equal to the direct sum of the (real) eigenspaces of $T$, and that $\operatorname{core}(T)$ contains the (unique) eigenvector of $T$ corresponding to 1 (namely, $e_{1}^{(3 r)}$ ) in its relative interior. Hence, we can find a proper cone $K_{r}$ of $\mathbb{R}^{3 r}$ invariant under $J_{r}(1) \oplus J_{r}\left(R_{\theta}\right)$ such that core $K_{r}\left(J_{r}(1) \oplus J_{r}\left(R_{\theta}\right)\right)$ is included in the direct sum of the (real) eigenspaces of $J_{r}(1) \oplus J_{r}\left(R_{\theta}\right)$ and contains the vector $e_{1}^{(3 r)}$ in its relative interior. In the above construction of $K_{r}$, by replacing the unit disc $D$ by $\alpha D$ for some suitable positive scalar $\alpha$, we can also ensure that core $_{K_{r}}\left(J_{r}(1) \oplus J_{r}\left(R_{\theta}\right)\right)$ is included in any given cone $C$ with the properties given in the lemma.

Now consider the case when $s>r$. From the first part of our proof, there exists a proper cone $K_{r}$ of $\mathbb{R}^{3 r}$ invariant under $J_{r}(1) \oplus J_{r}\left(R_{\theta}\right)$ such that 1 is a simple eigenvalue of the restriction of this matrix to its core (relative to $K_{r}$ ). By Lemma 7.4 we can find a rank-one matrix $y z^{T}$ such that the matrix

$$
\left[\begin{array}{cc}
J_{s-r}(1) & y z^{T} \\
0 & J_{r}(1) \oplus J_{r}\left(R_{\theta}\right)
\end{array}\right]
$$

is similar to $J_{s}(1) \oplus J_{r}\left(R_{\theta}\right)$, and leaves invariant the cone $\mathbb{R}_{+}^{s-r} \times K_{r}$, and that its core relative to this cone is equal to $\operatorname{ray}\left(e_{1}^{(2 r+s)}\right)$. So the existence of the desired proper invariant cone for $J_{s}(1) \oplus J_{r}\left(R_{\theta}\right)$ follows. 
Finally, suppose in addition that $\theta$ is a rational multiple of $\pi$. Then $\theta$ is an $n$th root of unity for some positive integer $n$. In this case, we modify our above construction of the proper cone $K$ by working with the polygon

$$
C_{n}=\operatorname{conv}\left\{(\cos 2 k \pi / n, \sin 2 k \pi / n)^{T}: k=0,1, \ldots, n-1\right\}
$$

(or its suitable positive multiple) instead of the unit disc $D$. The resulting cone $K$ obtained thus is always polyhedral.

Remark 7.7. In Lemma 7.6 if we replace $J_{r}\left(R_{\theta}\right)$ by $J_{r}(-1)$, the corresponding result still holds, subject to the following slight changes: $\mathbb{R}^{2 r+s}, e_{1}^{(2 r+s)}$ are replaced by $\mathbb{R}^{r+s}$ and $e_{1}^{(r+s)}$ respectively, $\operatorname{core}_{K}\left(J_{s}(1) \oplus J_{r}(-1)\right)$ is a 2-dimensional cone in case $r=s$, and $K$ can always be chosen to be polyhedral. The proof is similar.

Theorem 7.8. Let $A$ be an $n \times n$ real matrix. Then there exists a proper cone $K$ such that $A \in \pi(K)$ and $\operatorname{core}_{K}(A)$ is polyhedral if and only if $A$ satisfies the Perron-Schaefer condition and every eigenvalue in the peripheral spectrum of $A$ with the same index as that of $\rho(A)$ is equal to $\rho(A)$ times a root of unity.

Proof. The "only if" part follows from Corollary 7.2.

"If" part: We may assume that $A$ is in real canonical form. If $\rho(A)=0$, simply take $K$ to be $\mathbb{R}_{+}^{n}$. So we need only consider the case when $\rho(A)>0$. By normalizing $A$, we may assume that $\rho(A)=1$. For simplicity, write $\nu_{1}(A)$ as $\nu$. We may assume that the first $p(\geqq 1)$ blocks of $A$ all correspond to the eigenvalue 1 and with the first block of order $\nu$, each of the next $q(\geqq 0)$ blocks is either of order $\nu$ and corresponds to the eigenvalue -1 or is of order $2 \nu$ and corresponds to a pair of conjugate unimodular complex eigenvalues, each of the next further $r(\geqq 0)$ blocks is either of order less than $\nu$ and corresponds to the eigenvalue -1 or is of order less than $2 \nu$ and corresponds to a pair of conjugate unimodular complex eigenvalues, and the remaining $s \quad(\geqq 0)$ blocks correspond to eigenvalues with modulus less than one. Write $\mathbb{R}^{n}$ as $\bigoplus_{j=1}^{t} W_{j}$, where $t=p+q+r+1, W_{j}$ is the coordinate subspace corresponding to the $j$ th block for $1 \leqq j \leqq p+q+r$, and $W_{t}$ is the direct sum of the coordinate subspaces corresponding to the last $s$ blocks (which, of course, may not exist).

For each $j, 2 \leqq j \leqq p$, choose $K_{j}$ to be the nonnegative orthant of $W_{j}$. Then since $\left.A\right|_{W_{j}}$ can be represented by $J_{k}(1)$ for some $k$ (depending on $j$ ), by Corollary 4.3, the core of $\left.A\right|_{W_{j}}$ relative to $K_{j}$ is a single ray.

Consider any $j, p+1 \leqq j \leqq p+q$. Then the restriction of $A$ to $W_{1} \oplus W_{j}$ is similar either to $J_{\nu}(1) \oplus J_{\nu}\left(R_{\theta}\right)$ for some real number $\theta$ not an integral multiple of $\pi$, or to $J_{\nu}(1) \oplus J_{\nu}(-1)$. In the former case, since the index of $e^{i \theta}$ as an eigenvalue of $A$ is $\nu$, according to our hypothesis $e^{i \theta}$ is a root of unity. So by Lemma 7.6 we can find a proper polyhedral cone $K_{j}$ of $W_{1} \oplus W_{j}$ invariant under $A$ such that $\operatorname{core}_{K_{j}}(A)$ contains the eigenvector $e_{1}^{(n)}$, and is a 3-dimensional cone included in the direct sum of the (real) eigenspaces of $\left.A\right|_{W_{1} \oplus W_{j}}$. In the latter case, by Remark 7.7 we can also find a proper polyhedral cone $K_{j}$ of $W_{1} \oplus W_{j}$ invariant under $A$ such that $\operatorname{core}_{K_{j}}(A)$ contains $e_{1}^{(n)}$, and is a 2-dimensional cone included in the direct sum of the eigenspaces of $\left.A\right|_{W_{1} \oplus W_{j}}$.

Consider any $j, p+q+1 \leqq j \leqq p+q+r$. Then $\left.A\right|_{W_{1} \oplus W_{j}}$ is similar either to $J_{\nu}(1) \oplus J_{m}\left(R_{\theta}\right)$ or to $J_{\nu}(1) \oplus J_{m}(-1)$ for some real number $\theta$ which is not 
an integral multiple of $\pi$, and some positive integer $m<\nu$. In either case, by applying Lemma 7.6 or Remark 7.7, we can find a proper cone $K_{j}$ of $W_{1} \oplus W_{j}$ invariant under $A$ such that $\operatorname{core}_{K_{j}}(A)$ equals $\operatorname{ray}\left(e_{1}^{(n)}\right)$.

Finally, by Lemma 7.5 we can also find a proper polyhedral cone $K_{t}$ of $W_{1} \oplus W_{t}$ (where $t=p+q+r+1$ ) invariant under $A$ such that $\operatorname{core}_{K_{j}}(A)$ equals $\operatorname{ray}\left(e_{1}^{(n)}\right)$.

Now take $K$ to be the cone $\bigoplus_{j=2}^{p} K_{j} \oplus\left(K_{p+1}+K_{p+2}+\cdots+K_{p+q+r+1}\right)$. Clearly $K$ is a full cone of $\mathbb{R}^{n}$ invariant under $A$. Note that, for any $j$, $p+1 \leqq j \leqq p+q+r$, we have,

$$
\left[\operatorname{core}_{K_{p+1}}(A)+\cdots+\operatorname{core}_{K_{j}}(A)\right] \cap\left[-\operatorname{core}_{K_{j+1}}(A)\right]=\{0\} .
$$

By repeated applications of Lemma 7.3 it follows that $K_{p+1}+\cdots+K_{p+q+r+1}$ is a closed, pointed cone invariant under $A$ such that the core of $A$ relative to this cone equals the sum of the polyhedral cones $\operatorname{core}_{K_{j}}(A), p+1 \leqq j \leqq p+q+r+1$. But core $_{K}(A)$ is the direct sum of the rays core $_{K_{j}}(A), 2 \leqq j \leqq p$, and the core of $A$ relative to $K_{p+1}+\cdots+K_{p+q+r+1}$, hence it is polyhedral. The proof is complete.

Theorem 7.9. Let $A$ be an $n \times n$ real matrix. Then there exists a proper polyhedral cone $K$ such that $A \in \pi(K)$ if and only if $A$ satisfies the Perron-Schaefer condition, and every eigenvalue in the peripheral spectrum of $A$ is equal to $\rho(A)$ times a root of unity.

Proof. The "only if" part follows from [B-T, Theorem 2]. The "if" part follows from a modification of the proof of the "if" part of Theorem 7.8.

Theorem 7.10. Let $A$ be an $n \times n$ real matrix. Then there exists a proper cone $K$ such that $A \in \pi(K)$ and core $_{K}(A)$ is a single ray if and only if $\rho(A)>0$, and the Jordan form of $A$ has exactly one block of maximal order corresponding to $\rho(A)$, and the index of $\rho(A)$ is larger than that of every other eigenvalue in the peripheral spectrum of $A$.

Proof. The "only if" part follows readily from Theorem 7.1(ii), because we always have $\operatorname{core}_{K}(A) \supseteq M \cap K, \operatorname{span}(M \cap K)=M$ (where $M$ denotes the linear subspace as given in Theorem 7.1) and $\operatorname{dim} M$ is equal to the number of Jordan blocks of $A$ of order $\nu_{\rho(A)}(A)$ corresponding to the eigenvalues in its peripheral spectrum.

"If" part: There is no loss of generality in assuming that $\rho(A)=1$. We apply the construction given in the "if" part of the proof of Theorem 7.8. In this case $q=0$. If $A$ does not have blocks of order less than $\nu$ corresponding to 1 , then $p=1$ and our construction will yield a proper cone $K$ of $\mathbb{R}^{n}$ invariant under $A$ such that $\operatorname{core}_{K}(A)=\operatorname{ray}\left(e_{1}^{(n)}\right)$, and we are done. Otherwise, for each $j, 2 \leqq j \leqq p$, as we are going to show, it is possible to construct a proper cone $\widetilde{K}_{j}$ of $W_{1} \oplus W_{j}$ invariant under $A$ such that $\operatorname{core}_{\widetilde{K}_{j}}(A)=\operatorname{ray}\left(e_{1}^{(n)}\right)$. For each $j$, instead of taking $K_{j}$ to be the nonnegative orthant of $W_{j}$ as in the original construction, we take $K_{j}$ to be this $\widetilde{K}_{j}$. Then our method still works.

Consider any fixed $j, 2 \leqq j \leqq p$. Clearly $\left.A\right|_{W_{1} \oplus W_{j}}$ is similar to $J_{\nu}(1) \oplus J_{k}(1)$ for some positive integer $k=k(j)<\nu$. For each positive integer $l, 1 \leqq l \leqq k$, let $P_{l}$ denote the cone generated by $e_{1}^{(\nu+k)}, e_{\nu+l}^{(\nu+k)}+e_{\nu}^{(\nu+k)}$ and the images of 
$e_{\nu+l}^{(\nu+k)}+e_{\nu}^{(\nu+k)}$ under the action of all positive powers of $J_{\nu}(1) \oplus J_{k}(1)$. Since

$$
\left[J_{\nu}(1) \oplus J_{k}(1)\right]^{i}\left(e_{\nu+l}^{(\nu+k)}+e_{\nu}^{(\nu+k)}\right) /\left\|\left[J_{\nu}(1) \oplus J_{k}(1)\right]^{i}\left(e_{\nu+l}^{(\nu+k)}+e_{\nu}^{(\nu+k)}\right)\right\|
$$

tends to $e_{1}^{(\nu+k)}$ as $i \rightarrow \infty$, it is clear that $P_{l}$ is a closed, pointed cone invariant under $A$. Noting the above limit, and the fact that for each positive integer $i$,

$$
\begin{aligned}
& {\left[J_{\nu}(1) \oplus J_{k}(1)\right]^{i} P_{l}} \\
& \quad=\operatorname{pos}\left\{e_{1}^{(\nu+k)},\left[J_{\nu}(1) \oplus J_{k}(1)\right]^{m}\left(e_{\nu+l}^{(\nu+k)}+e_{\nu}^{(\nu+k)}\right), m=i, i+1, \ldots\right\},
\end{aligned}
$$

it is not difficult to show that $\operatorname{core}_{P_{l}}\left(J_{\nu}(1) \oplus J_{k}(1)\right)=\operatorname{ray}\left(e_{1}^{(\nu+k)}\right)$. Now let $C_{j}$ be the sum of the cones $P_{l}, 1 \leqq l \leqq k$, and the cone $\operatorname{pos}\left\{e_{1}^{(\nu+k)}, e_{2}^{(\nu+k)}, \ldots, e_{\nu}^{(\nu+k)}\right\}$. Since $e_{1}^{(\nu+k)}, \ldots, e_{\nu}^{(\nu+k)}, e_{\nu+1}^{(\nu+k)}+e_{\nu}^{(\nu+k)}, \ldots, e_{\nu+k}^{(\nu+k)}+e_{\nu}^{(\nu+k)}$ form a basis of $\mathbb{R}^{\nu+k}$, clearly $C_{j}$ is a full cone of $\mathbb{R}^{\nu+k}$. An application of Lemma 7.3 shows that $C_{j}$ is in fact a proper cone of $\mathbb{R}^{\nu+k}$ invariant under $J_{\nu}(1) \oplus J_{k}(1)$ such that core $_{C_{j}}\left(J_{\nu}(1) \oplus J_{k}(1)\right)=\operatorname{ray}\left(e_{1}^{(\nu+k)}\right)$. Since $\left.A\right|_{W_{1} \oplus W_{j}}$ is similar to $J_{\nu}(1) \oplus J_{k}(1)$, the desired cone $\widetilde{K}_{j}$ clearly exists. The proof is complete.

In [Schn 1, Corollary 5.3] Schneider showed that if $A \in \pi(K)$ then $K$ contains at least $p$ linearly independent eigenvectors of $A$ corresponding to $\rho(A)$, where $p$ is the number of Jordan blocks of $A$ of order $\nu_{\rho(A)}(A)$. Our next result implies that this lower bound for the number of linearly independent eigenvectors is best possible.

Theorem 7.11. Let $A$ be an $n \times n$ real matrix that satisfies the Perron-Schaefer condition. Let $p$ be the number of blocks in the Jordan form of $A$ of maximal order that correspond to $\rho(A)$. Then for each positive integer $k, p \leqq k \leqq$ $\operatorname{dim} \mathfrak{N}(\rho(A) I-A)$, there exists a proper cone $K$ of $\mathbb{R}^{n}$ such that $A \in \pi(\bar{K})$ and $\mathfrak{N}(\rho(A) I-A) \cap K$ is a cone of dimension $k$.

Proof. When $\rho(A)>0$, the desired cone $K$ can be constructed by the method given in the proofs of the "if" part of Theorems 7.8 and 7.10.

Now consider the case when $\rho(A)=0$. We may assume that $A$ is in Jordan canonical form. It suffices to show that if $A$ has exactly one block of maximal order then we can construct a proper cone $K$ invariant under $A$ such that $\mathfrak{N}(A) \cap K$ is of dimension 1. (The general case then follows by a direct sum argument.) Suppose that the first block of $A$ is of maximal order, say of order $\nu$. It is straightforward to verify that the vectors $e_{1}^{(n)}, \ldots, e_{\nu}^{(n)}, e_{\nu+1}^{(n)}+e_{\nu}^{(n)}$, $e_{\nu+2}^{(n)}+e_{\nu}^{(n)}, \ldots, e_{n}^{(n)}+e_{\nu}^{(n)}$ form a basis of $\mathbb{R}^{n}$. Let $K$ be the cone generated by these basis vectors together with their images under the action of all positive powers of $A$. Since $A$ is nilpotent, clearly $K$ (being finitely generated) is polyhedral. $K$ is also pointed, because $K \subseteq \mathbb{R}_{+}^{n}$. Thus $K$ is a proper cone of $\mathbb{R}^{n}$, invariant under $A$. Also, it is not difficult to show that $\mathfrak{N}(A) \cap K=$ $\operatorname{ray}\left(e_{1}^{(n)}\right)$.

Now we treat the question of determining a necessary and sufficient condition on an $n \times n$ real matrix $A$ for which there exists a proper cone $K$ of $\mathbb{R}^{n}$ such that $A \in \pi(K)$ and $\operatorname{core}_{K}(A)$ is a nonzero simplicial cone. As we shall see, in this case, our equivalent condition depends not only on eigenvalues in the peripheral spectrum of $A$ with the same index as that of $\rho(A)$, but also on other 
eigenvalues in the peripheral spectrum. Just for convenience, we normalize our matrix $A$ and assume that $\rho(A)=1$.

Theorem 7.12. Let $A$ be an $n \times n$ real matrix with $\rho(A)=1$ that satisfies the Perron-Schaefer condition. Let $S$ be the multi-set of eigenvalues in the peripheral spectrum of $A$ with index equal to $\nu_{1}(A)$, the multiplicity of each number being equal to the number of corresponding blocks in the Jordan form of $A$ of order $\nu_{1}(A)$. Let $T$ be the multi-set of eigenvalues in the peripheral spectrum of $A$ for which there are corresponding blocks in the Jordan form of $A$ of order less than $\nu_{1}(A)$, the multiplicity of each number being equal to the number of such blocks. For each positive integer $m$, denote by $Z_{m}$ the set of all mth roots of unity. Then there exists a proper cone $K$ in $\mathbb{R}^{n}$ such that $A \in \pi(K)$ and core $_{K}(A)$ is a simplicial cone if and only if there is a (possibly empty) multi-subset $\widetilde{T}$ of $T$ such that the multi-set union of $S$ and $\widetilde{T}$ is the same as the multi-set union of certain $Z_{m}$ 's.

Before we come to the proof, an example is in order. Let $A$ be a $7 \times 7$ real matrix whose Jordan form is $J_{2}(1) \oplus J_{1}(1) \oplus J_{2}(-1) \oplus J_{2}(-1)$. In the notation of our theorem, the multi-sets $S$ and $T$ in this case are respectively $\{1,-1,-1\}$ and $\{1\}$. So their multi-set union is $\{1,1,-1,-1\}$ which is the same as the multi-set union of $Z_{2}$ and $Z_{2}$. According to our theorem, there exists a proper cone $K$ of $\mathbb{R}^{7}$ such that $A \in \pi(K)$ and core $_{K}(A)$ is a simplicial cone.

Proof of Theorem 7.12. "Only if" part: Suppose that there exists a proper cone $K$ in $\mathbb{R}^{n}$ such that $A \in \pi(K)$ and $\operatorname{core}_{K}(A)$ is a simplicial cone. Denote by $M$ the intersection of $\mathbb{R}^{n}$ with the subspace $\bigoplus_{\lambda}\left[(\lambda I-A)^{\nu-1} \mathfrak{N}\left((\lambda I-A)^{\nu}\right)\right]$, where the direct sum runs through all unimodular eigenvalues of $A$ with index $\nu=\nu_{1}(A)$. Then by Theorem 7.1(ii) $M=\operatorname{span}\left(M \cap \operatorname{core}_{K}(A)\right)$. Since $\left.A\right|_{M}$ is diagonalizable, all eigenvalues of $\left.A\right|_{M}$ are of modulus one and 1 is an eigenvalue of $\left.A\right|_{M}$, by Theorem $5.9(\mathrm{a}) \Rightarrow$ (d) $A$ has an eigenvector in $\operatorname{relint}(M \cap \operatorname{core}(A))$, say $x$, corresponding to the eigenvalue 1 . For any subset $S$ of $\operatorname{core}(A)$, denote by $\widetilde{\Phi}(S)$ the face of $\operatorname{core}(A)$ generated by $S$. Then $\widetilde{\Phi}(M \cap \operatorname{core}(A))=\widetilde{\Phi}(x)$ and is an $A$-invariant face of $\operatorname{core}(A)$, because $x$ is an eigenvector of $A$; hence by Lemma 3.7, $A(\widetilde{\Phi}(M \cap \operatorname{core}(A))=\widetilde{\Phi}(M \cap \operatorname{core}(A))$. For convenience, denote the restriction of $A$ to $\operatorname{span}[\widetilde{\Phi}(M \cap \operatorname{core}(A))]$ by $B$. Note that $x$ also lies in relint $\widetilde{\Phi}(M \cap \operatorname{core}(A))$, so by Theorem $5.9(\mathrm{~b}) \Rightarrow(\mathrm{a}), B$ is diagonalizable, and all of its eigenvalues are of modulus one. Hence the spectrum of $B$ (as a multiset) is included in the union of the multi-sets $S$ and $T$ (which is in fact the multi-set of all eigenvalues in the peripheral spectrum of $A$, the multiplicity of each number being equal to its geometric multiplicity as an eigenvalue of $A$ ). Note also that the spectrum of $B$ includes the spectrum of $\left.A\right|_{M}$ (as $M=$ $\operatorname{span}(M \cap \operatorname{core}(A)))$, and that the latter spectrum is in turn equal to the multi-set $S$. On the other hand, as a face of the simplicial cone $\operatorname{core}(A), \widetilde{\Phi}(M \cap \operatorname{core}(A))$ is itself a simplicial cone. Since $B$ takes this latter cone onto itself and the eigenvalues of $B$ are all of modulus one, it follows that $B$ is similar to a direct sum of nonnegative monomial matrices, each of which has spectral radius one, and hence the spectrum of $B$ is the multi-set union of certain $Z_{m}$ 's. Thus the existence of the desired multi-subset $\widetilde{T}$ of $T$ follows.

"If" part: We need treat only the case when $A$ has exactly one Jordan block of order $\nu=\nu_{1}(A)$ corresponding to the eigenvalue 1. The general case then follows by a direct sum argument. 
Suppose that there is a multi-subset $\widetilde{T}$ of $T$ such that the multi-set $S \cup$ $\tilde{T}$ is equal to the multi-set union of $Z_{r_{1}}, \ldots, Z_{r_{s}}$ for some positive integers $r_{1}, \ldots, r_{s}$. By a real similarity transformation, we may assume that $A$ is of the form $A_{1} \oplus \cdots \oplus A_{s+1}$ where $\rho\left(A_{i}\right)=1$ for $1 \leqq i \leqq s$ and the following conditions are satisfied: (i) For each $i, 1 \leqq i \leqq s$, the set of eigenvalues of $A_{i}$ is equal to $Z_{r_{i}}$ (not counting multiplicities) and $A_{i}$ has exactly one Jordan block corresponding to each of its eigenvalues; (ii) The index of each unimodular eigenvalue, if any, of the matrix $A_{s+1}$ (which may not exist at all) is less than $\nu$; and (iii) $\nu_{1}\left(A_{1}\right)=\nu>\nu_{1}\left(A_{i}\right)$ for $2 \leqq i \leqq s$.

Denote by $W_{i}$ the coordinate subspace of $\mathbb{R}^{n}$ corresponding to the block $A_{i}$ of $A$ for $i=1, \ldots, s+1$. Denote by $U$ the direct sum of the (real) eigenspaces of the restriction map $\left.A\right|_{\oplus_{i=1}^{s} W_{i}}$. Also let $u$ be the (unique) eigenvector of $A$ in $W_{1}$ that corresponds to the eigenvalue 1 . Then $\left.A\right|_{U}$ is similar to the matrix $\bigoplus_{i=1}^{s} P_{r_{i}}$, where we use $P_{r}$ do denote the $r \times r$ permutation matrix given by

$$
P_{r}=\left[\begin{array}{llllll}
0 & & & & & 1 \\
1 & 0 & & & & \\
& 1 & . & & & \\
& & \cdot & . & & \\
& & & \cdot & . & \\
& & & & 1 & 0
\end{array}\right] .
$$

Furthermore, there exists a proper $A$-invariant simplicial cone $C$ of $U$ that contains $u$ in its relative interior. [Indeed, if $T$ is an isomorphism between $\mathbb{R}^{m}$ and $U$, where $m=\sum_{i=1}^{s} r_{i}$, that takes $\sum_{i=1}^{m} e_{i}^{(m)}$ to $u$, and every (real) eigenspace of $\bigoplus_{i=1}^{S} P_{r_{i}}$ to the corresponding (real) eigenspace of $\left.A\right|_{U}$, then $T^{-1}\left(\left.A\right|_{U}\right) T=\bigoplus_{i=1}^{s} P_{r_{i}}$ and the desired cone $C$ can be taken to be $T \mathbb{R}_{+}^{m}$.] Note that $A C=C$.

For each $i, 1 \leqq i \leqq s$, and for each conjugate pair of nonreal complex eigenvalues $\lambda, \bar{\lambda}$ of $A_{i}$, by Lemma 7.6 we can construct an $A$-invariant cone $K_{i \lambda}$ which is a proper cone in the direct sum of the generalized eigenspace of $\left.A\right|_{W_{1}}$ corresponding to the eigenvalue 1 and the real generalized eigenspace of $\left.A\right|_{W_{i}}$ corresponding to the conjugate pair $\lambda, \bar{\lambda}$ such that core $_{K_{i \lambda}}(A)$ equals $\operatorname{ray}(u)$ if $\nu_{\lambda}(A)<\nu$, or is a (3-dimensional) proper cone of the direct sum of the corresponding (real) eigenspaces that contains $u$ in its relative interior, if $\nu_{\lambda}(A)=\nu$. Indeed, because $u \in \operatorname{relint} C$, when $\nu_{\lambda}(A)=\nu$ by the same lemma the cone $K_{i \lambda}$ can also be constructed in such a way that core $_{K_{i \lambda}}(A) \subseteq C$. For each $i, 1 \leqq i \leqq s$, also construct a similar cone $K_{i,-1}$ for the eigenvalue -1 of $\left.A\right|_{W_{i}}$, if it exists.

For each $i, 2 \leqq i \leqq s$, by the proof of the "if" part of Theorem 7.10, we can construct an $\bar{A}$-invariant cone $K_{i}$ which is a proper cone in the direct sum of the generalized eigenspace of $\left.A\right|_{W_{1}}$ corresponding to the eigenvalue 1 and that of $\left.A\right|_{W_{i}}$ corresponding to the same eigenvalue such that core $_{K_{i}}(A)$ equals $\operatorname{ray}(u)$. Finally, also construct an $A$-invariant cone $K_{s+1}$ which is a proper cone in the direct sum of the generalized eigenspace of $\left.A\right|_{W_{1}}$ corresponding to the eigenvalue 1 and the coordinate subspace $W_{s+1}$ such that $\operatorname{core}_{K_{s+1}}(A)$ equals $\operatorname{ray}(u)$.

Now take $K$ to be the sum of the cone $C$ and all the cones $K_{i \lambda}$ and $K_{i}$ constructed above. Using Lemma 7.3 it is not difficult to show that $K$ is a 
proper cone of $\mathbb{R}^{n}$ invariant under $A$ such that $\operatorname{core}_{K}(A)$ equals the simplicial cone $C$. The proof is complete.

In this section, Lemmas 7.3 and 7.4 have played an important role in our construction of invariant cones $K$ for a matrix $A$ with the Perron-Schaefer condition such that $\operatorname{core}_{K}(A)$ satisfies certain properties. So far, in our construction, $\operatorname{core}_{K}(A)$ is always included in the direct sum of (real) eigenspaces of $A$ corresponding to the eigenvalues in its peripheral spectrum. By a direct sum argument, it is clear that $K$ can be chosen in such a way that any nonnegative eigenvalue can be a distinguished eigenvalue of $A$ for $K$. By Corollary 7.14 below one can also choose $K$ so that core $_{K}(A)$ contains generalized eigenvectors of $A$ of order two or more. We omit the details.

Theorem 7.13. Let $\lambda$ be a positive real number. For each positive integer $n$, there exists a proper cone $K$ of $\mathbb{R}^{n}$ such that $J_{n}(\lambda) \in \operatorname{Aut}(K)$ if and only if $n$ is odd.

Proof. In view of $D^{-1}\left[J_{n}(\lambda) / \lambda\right] D=J_{n}(1)$, where $D=\operatorname{diag}\left(1, \lambda, \ldots, \lambda^{n-1}\right)$, it suffices to consider the special case when $\lambda=1$.

"Only if" part: Assume to the contrary that for some even positive integer $n$ and some proper cone $K$ of $\mathbb{R}^{n}$, we have, $J_{n}(1) \in \operatorname{Aut}(K)$. Since $K$ is full, we can choose a vector $x$ from $K$ with a nonzero $n$th component, say it is positive. Straightforward calculations yield $\lim _{k \rightarrow \infty} J_{n}(1)^{k} x /\left\|J_{n}(1)^{k} x\right\|=e_{1}^{(n)}$ and $\lim _{k \rightarrow \infty} J_{n}(1)^{-k} x /\left\|J_{n}(1)^{-k} x\right\|=-e_{1}^{(n)}$. In view of the closedness of $K$, we have, $e_{1}^{(n)} \in(-K) \cap K$, which contradicts the pointedness of $K$.

"If" part: When $n=1$ our assertion holds trivially. Consider an odd integer $n$ greater than 1. For any integer $k$ and any positive integer $r$, let $\left(\begin{array}{l}k \\ r\end{array}\right)$ denote the number $k(k-1) \cdots(k-r+1) / r$ !. Let $C$ be the unbounded convex set with extreme points

$$
x_{k}=\left(\left(\begin{array}{c}
k \\
n-1
\end{array}\right),\left(\begin{array}{c}
k \\
n-2
\end{array}\right), \ldots,\left(\begin{array}{l}
k \\
1
\end{array}\right)\right)^{T}, \quad k=0, \pm 1, \pm 2, \ldots,
$$

and recession cone $O^{+} C=\operatorname{ray}\left(e_{1}^{(n-1)}\right)$. As can be shown $C$ is a closed convex set, with nonempty interior. Let $K$ be the proper cone of $\mathbb{R}^{n}$ given by: $K=$ $\left\{\alpha\left(\begin{array}{l}x \\ 1\end{array}\right): \alpha \geqq 0, x \in C\right\} \cup\left(O^{+} C \times\{0\}\right)$. For each integer $k$, note that the point $\left(\begin{array}{c}x_{k} \\ 1\end{array}\right)$ is in fact $J_{n}(1)^{k} e_{n}^{(n)}$. Thus $J_{n}(1)$ takes the convex set $C_{n} \times\{1\}$ onto itself, and so $J_{n}(1) \in \operatorname{Aut}(K)$.

Corollary 7.14. Let $n$ be a given positive integer. Then for each odd positive integer $d \leqq n$, there exists a proper cone $K$ of $\mathbb{R}^{n}$ such that $J_{n}(1) \in \pi(K)$ and core $_{K}\left(J_{n}(1)\right)$ is a cone of dimension $d$.

Proof. Let $d$ be an odd positive integer less than or equal to $n$. By Theorem 7.13 there exists a proper cone $K_{d}$ of $\mathbb{R}^{d}$ such that $J_{d}(1) \in \operatorname{Aut}\left(K_{d}\right)$. By Lemma 7.4 there exists a rank-one matrix $y z^{T}$ such that the matrix

$$
\left[\begin{array}{cc}
J_{d}(1) & y z^{T} \\
0 & J_{n-d}(1)
\end{array}\right]
$$

is similar to $J_{n}(1)$, leaves invariant the cone $K_{d} \times \mathbb{R}_{+}^{n-d}$, and its core relative to $K_{d} \times \mathbb{R}_{+}^{n-d}$ is the $d$-dimensional cone $K_{d} \times\{0\}$. So our result follows. 
Finally, one may also ask the question of determining an equivalent condition on an $n \times n$ real matrix $A$ for which there exists a proper cone $K$ of $\mathbb{R}^{n}$ such that $A \in \pi(K)$ and core $_{K}(A)$ is a full cone. As can be readily seen, this question is equivalent to asking when there exists a proper cone $K$ such that $A \in \operatorname{Aut}(K)$. This question seems more difficult and will form the subject matter of our future work [Tam 2].

\section{THE COMPLEX CASE}

In this paper we restrict our attention to cones in a real vector space, because "cone" is a real concept. Schneider [Schn 1; see the discussion preceding Corollary 5.3] has shown that if $A$ is an $n \times n$ complex matrix which leaves invariant a proper cone of $\mathbb{C}^{n}$ then $A$ satisfies the Perron-Schaefer condition. Here we treat $\mathbb{C}^{n}$ as a real vector space (of dimension $2 n$ ) and borrow the definition of a proper cone from the real case. It is natural to ask whether the results in this paper still hold for a complex matrix preserving a proper cone of $\mathbb{C}^{n}$. As we are going to explain, to a great extent, the answer to this question is in the affirmative.

Let $A$ be an $n \times n$ complex matrix, and let $K$ be a proper cone of $\mathbb{C}^{n}$ such that $A K \subseteq K$. As before, we can define $\operatorname{core}_{K}(A)$ to be the set $\bigcap_{i=1}^{\infty} A^{i} K$. Treating $A$ as a real linear transformation on $\mathbb{C}^{n}$ (as a real vector space), we can apply Theorem 2.2 to deduce that $\operatorname{core}_{K}(A)$ is a closed, pointed cone, and that $A\left[\operatorname{core}_{K}(A)\right]=\operatorname{core}_{K}(A)$. Also, by Theorem $2.4 \operatorname{core}_{K}(A)$ is a polyhedral cone, whenever $K$ is. Since the definitions of a distinguished eigenvalue, a distinguished eigenvector, and $A$-invariant face, the nullspace of $A$, etc. depend only on the property of $A$ as a real linear transformation, many results in this paper a priori hold also in the complex case; for instance, Theorem 3.2, Lemmas 3.7, 3.9, 5.2 and Corollary 3.15 , to mention just a few. Because "cone" is a real concept, for a result or a concept in which a linear span is involved, to extend it to the complex case, we usually take the span to be a real span. With this slight modification, Theorem 3.14 and Lemma 7.3 can also be carried over to the complex case.

To extend results that involve the spectrum of $A$, care must be taken; because the spectrum of $A$ as a complex linear transformation and that of $A$ as a real linear transformation are not the same. Fortunately, the two spectra are related in a simple way, as indicated in the following result.

Lemma 8.1. Let $A$ be an $n \times n$ complex matrix. Treated as a real linear transformation acting on $\mathbb{C}^{n}, A$ is similar to the matrix $\operatorname{diag}(A, \bar{A})$, where $\bar{A}$ denotes the conjugate matrix of $A$.

Proof. Write $A$ as $C+i D$, where $C, D$ are $n \times n$ real matrix. For any vectors $x, y \in \mathbb{R}^{n}$, we have, $A(x+i y)=(C x-D y)+i(D x+C y)$. Denote by $\eta: \mathbb{C}^{n} \rightarrow \mathbb{R}^{2 n}$ the canonical real isomorphism given by $\eta(x+i y)=\left(\begin{array}{l}x \\ y\end{array}\right)$. Then it can be easily checked that $\eta \circ A=\tilde{A} \circ \eta$, where $\tilde{A}$ is the $2 n \times 2 n$ real matrix $\left[\begin{array}{cc}C & -D \\ D & C\end{array}\right]$. So $A$ (as a real linear transformation) is similar to the real matrix $\widetilde{A}$. But $\widetilde{A}$ is, in turn, similar to the matrix $\operatorname{diag}(A, \bar{A})$, because we have $P^{-1} \tilde{A} P=\operatorname{diag}(A, \bar{A})$, where $P$ is the $2 n \times 2 n$ nonsingular matrix given 
by

$$
P=\left[\begin{array}{cc}
I_{n} & -i I_{n} / 2 \\
-i I_{n} & I_{n} / 2
\end{array}\right]
$$

Hence, our assertion follows.

It is clear that if a complex matrix $A$ satisfies the Perron-Schaefer condition then so does the matrix $\operatorname{diag}(A, \bar{A})$, and conversely. In view of Lemma 8.1 , it follows that an $n \times n$ complex matrix $A$ leaves invariant some proper cone of $\mathbb{C}^{n}$ if and only if $A$ satisfies the Perron-Schaefer condition. (This completes the observation made by Schneider mentioned at the beginning of this section.)

Lemma 8.1 can also be used to deduce readily the complex analogs of many other results in this paper, including Theorems 5.4, 5.9, 7.8, 7.9, etc. For an $n \times n$ complex matrix $A$, the necessary and sufficient condition for the existence of a proper $A$-invariant cone $K$ of $\mathbb{C}^{n}$ such that $\operatorname{core}_{K}(A)$ is simplicial is not exactly the one given in Theorem 7.12. The condition given there has to be modified: consider the peripheral spectrum of $\operatorname{diag}(A, \bar{A})$ instead of that of $A$. For instance, by this modified condition, the matrix $J_{1}(1) \oplus J_{1}(-1) \oplus J_{1}(i)$ has an invariant proper cone in $\mathbb{C}^{3}$ such that its core relative to this cone is simplicial.

The complex analog of Theorem 6.3 also holds. This can be seen as follows. Consider a proper cone $K$ of $\mathbb{C}^{n}$ and an $n \times n$ complex matrix $A \in \pi(K)$. Let $\lambda$ be a nonzero distinguished eigenvalue of $A$. Also let $\mu$ be a nonreal eigenvalue of $A$ with the same modulus as $\lambda$. Then the direct sum $\mathfrak{N}(\mu I-A)$ $\oplus \mathfrak{N}(\bar{\mu} I-A)$ is equal to the real eigenspace of $A$ (when treated as a real linear transformation) corresponding to the conjugate pair $\mu, \bar{\mu}$. (Here, of course, $\mathfrak{N}(\bar{\mu} I-A)$ is the zero subspace if $\bar{\mu}$ is not an eigenvalue of $A$ as a complex matrix.) Indeed, both spaces are equal to $\mathfrak{N}\left(\left(a^{2}+b^{2}\right) I-2 a A+A^{2}\right)$, where $a, b \in \mathbb{R}, b \neq 0$ such that $\lambda=a+i b$. With this in mind, it is clear that the set $W_{\lambda}$ for $A$ as a real linear transformation is equal to the direct sum of all eigenspaces of $A$ (as a complex matrix) corresponding to eigenvalues with modulus $\lambda$. So Theorem 6.3 can be carried over to the complex case.

Similarly, it is also true that if $A$ is an $n \times n$ complex matrix and $\mu$ is a nonreal eigenvalue of $A$ then the direct sum of the generalized eigenspaces of $A$ corresponding to $\mu$ and $\bar{\mu}$ (if $\bar{\mu}$ is not an eigenvalue, the corresponding generalized eigenspace is the zero subspace) is equal to the real generalized eigenspace of $A$ (treated as a real linear transformation) corresponding to the conjugate pair $\mu, \bar{\mu}$. So Theorem 5.10 can also be extended to the complex case, if we take $M$ to be the direct sum of the generalized eigenspaces of $A$ corresponding to eigenvalues in its peripheral spectrum.

The complex analog of Theorem 7.1 also holds, since the original proof works.

One special feature about cone-preserving maps is that the important concepts of irreducibility, primitivity and strict positivity cannot be carried over to the complex case in the natural way. (This is also another reason why the theory of complex cone-preserving maps is less interesting.) The point is, there do not exist a proper cone $K$ of $\mathbb{C}^{n}(n \geqq 1)$ and an $n \times n$ complex matrix $A \in \pi(K)$ such that $A$ has (up to nonnegative multiples) a unique eigenvector that lies in int $K$; this is because, if $u \in$ int $K$ is an eigenvector of $A$ then in the real linear span generated by $u$ and $i u$, we can find another eigenvector of $A$ that lies in int $K$ and is not a nonnegative multiple of $u$. So the results 
we obtain in $\S 5$ about the primitivity or irreducibility of cone-preserving maps cannot be extended to the complex case. By the above reasoning (or by Lemma 8.1 and Theorem 7.10) it is clear that there do not exist a proper cone $K$ of $\mathbb{C}^{n}$ and an $n \times n$ complex matrix $A \in \pi(K)$ such that core ${ }_{K}(A)$ is a single ray. Thus Lemma 5.3 cannot be extended to the complex case, since its conclusion cannot be satisfied at all. Lemma 3.14 also has no complex analog, because its hypothesis cannot be satisfied in the complex case.

\section{ACKNOWLEDGMENT}

The first author is glad to acknowledge having fruitful discussions with D. Ž. Djoković on an earlier version of this paper.

\section{REFERENCES}

[Bar 1] G. P. Barker, On matrices having an invariant cone, Czechoslovak Math. J. 22 (1972), 49-68.

[Bar 2] _ Theory of cones, Linear Algebra Appl. 39 (1981), 263-291.

[B-N] R. Bru and M. Neumann, Nonnegative Jordan bases, Linear and Multilinear Algebra 23 (1988), 95-109.

[B-N-S] A. Berman, M. Neumann, and R. J. Stern, Nonnegative matrices in dynamical system, J. Wiley, New York, 1989.

[B-P] A. Berman and R. J. Plemmons, Nonnegative matrices in the mathematical sciences, Academic Press, New York, 1979.

[B-S] G. P. Barker and H. Schneider, Algebraic Perron-Frobenius theory, Linear Algebra Appl. 11 (1975), 219-233.

[B-T] G. P. Barker and R. E. L. Turner, Some observations on the spectra of cone-preserving maps, Linear Algebra Appl. 6 (1973), 149-153.

[B-T-D] G. P. Barker, B. S. Tam, and N. Davila, A geometric Gordan-Stiemke theorem, Linear Algebra Appl. 61 (1984), 83-89.

[Bir] G. Birkhoff, Linear transformations with invariant cones, Amer. Math. Monthly 74 (1967), 274-276.

[Bon] F. F. Bonsall, Sublinear functionals and ideals in partially ordered vector spaces, Proc. London Math. Soc. 4 (1954), 402-418.

[Dok] D. Ž. Djoković, Quadratic cones invariant under some linear operators, SIAM J. Algebraic Discrete Methods 8 (1987), 186-191.

[Ell] A. J. Ellis, On faces of compact convex sets and their annihilators, Math. Ann. 184 (1969), 19-24.

[Els 1] L. Elsner, Monotonie und Randspektrum bei vollstetigen Operatoren, Arch. Rational Mech. Anal. 36 (1970), 356-365.

[Els 2] __ On matrices leaving invariant a nontrivial convex. set, Linear Algebra Appl. 42 (1982), 103-107.

[Fri 1] S. Friedland, Characterizations of spectral radius of positive operators, Linear Algebra Appl. 134 (1990), 93-105.

[Fri 2] _ Characterizations of spectral radius of positive operators on $C^{*}$ algebras, J. Funct. Anal. 97 (1991), 64-70.

[Fro] G. F. Frobenius, Uber Matrizen aus nicht negativen Elementen, Sitzungsber. Kon. Preuss Akad. Wiss. Berlin, (1912), 456-477; Ges. Abh. 3 (1968), 546-567. 
[G-L-R] I. Gohberg, P. Lancaster, and L. Rodman, Invariant subspaces of matrices with applications, J. Wiley, New York, 1986.

[Gro] U. Groh, Some observations on the spectra of positive operators on finite-dimensional $C^{*}$ algebras, Linear Algebra Appl. 42 (1982), 213-222.

[H-S 1] D. Hershkowitz and H. Schneider, Height bases, level bases, and the equality of height and level characteristics of an M-matrix, Linear and Multilinear Algebra 25 (1989), 149-171.

[H-S 2] __ Combinatorial basis, derived Jordan sets and the equality of the height and the level characteristics of an M-matrix, Linear and Multilinear Algebra 29 (1991), 21-42.

[H-S 3] __, On the existence of matrices with prescribed height and level characteristics, Israel J. Math. 75 (1991), 105-117.

[H-W] G. H. Hardy and E. M. Wright, An introduction to the theory of numbers, 4th ed., Oxford Univ. Press, London, 1960.

[Hor] J. G. Horne, On the automorphism group of a cone, Linear Algebra Appl. 21 (1978), 111-121.

[J-V] R. J. Jang and H. D. Victory, Jr., On nonnegative solvability of linear integral equations, preprint.

[K-R] M. G. Krein and M. A. Rutman, Linear operators leaving invariant a cone in a Banach space, Amer. Math. Soc. Transl. Ser. (1) 10 (1962), 199-325 [originally Uspekhi Mat. Nauk (N.S.) 3 (1948), 3-95].

[L-T] P. Lancaster and M. Tismenetsky, The theory of matrices, 2nd ed., Academic Press, New York, 1985.

[Min] H. Minc, Nonnegative matrices, Wiley, New York, 1988.

[Nel] P. Nelson, Jr., The structure of a positive linear integral operator, J. London Math. Soc. (2) 8 (1974), 711-718.

[Pul] N. J. Pullman, A geometric approach to the theory of nonnegative matrices, Linear Algebra Appl. 4 (1971), 297-312.

[Roc] R. T. Rockafellar, Convex analysis, Princeton Univ. Press, Princeton, NJ, 1970.

[Rot] U. G. Rothblum, Algebraic eigenspaces of nonnegative matrices, Linear Algebra Appl. 12 (1975), 281-292.

[Scha 1] H. H. Schaefer, Banach lattices and positive operators, Springer, New York, 1974.

[Scha 2] _ , Topological vector spaces, 4th printing, Springer, New York, 1980.

[Scha 3] __ A metric variant of Frobenius theorem and some other remarks on positive matrices, Linear Algebra Appl. 42 (1982), 175-182.

[Schn 1] H. Schneider, Geometric conditions for the existence of positive eigenvalues of matrices, Linear Algebra Appl. 38 (1981), 253-271.

[Schn 2] _ The influence of the marked reduced graph of a nonnegative matrix on the Jordan form and on related properties: a survey, Linear Algebra Appl. 84 (1986), 161-189.

[S-W 1] R. Stern and H. Wolkowicz, Invariant ellipsoidal cones, Linear Algebra Appl. 150 (1991), 81-106.

[S-W 2] _ A note on generalized invariant cones and the Kronecker canonical form, Linear Algebra Appl. 147 (1991), 97-100.

[Tam 1] B. S. Tam, On the distinguished eigenvalues of a cone-preserving map, Linear Algebra Appl. 131 (1990), 17-37.

[Tam 2] _ On matrices with invariant proper cones, (in preparation).

[T-S] B. S. Tam and H. Schneider, On the invariant faces of a cone-preserving map, (in preparation).

[T-W] B. S. Tam and S. F. Wu, On the Collatz-Wielandt sets associated with a cone-preserving map, Linear Algebra Appl. 125 (1989), 77-95.

[Van] J. S. Vandergraft, Spectral properties of matrices which have invariant cones, SIAM J. Appl. Math. 16 (1968), 1208-1222.

[V-L] A. I. Veitsblit and Yu. I. Lyubich, Boundary spectrum of nonnegative operators, Siberian Math. J. 26 (1985), 798-802 [translated from Sibirsk. Mat. Zh. 26 (1985), 24-28].

[Vic] H. D. Victory, Jr., On linear integral operators with nonnegative kernels, J. Math. Anal. Appl. 29 (1982), 420-441. 
[Vic 2] _ The structure of the algebraic eigenspace to the spectral radius of eventually compact, nonnegative integral operators, J. Math. Anal. Appl. 90 (1982), 484-516.

[Vic 3] __ On nonnegative solutions to matrix equations, SIAM J. Algebraic Discrete Methods 6 (1985), 406-412.

Department of Mathematics, Tamkang University, Tamsui, Taiwan 25137, R.O.C. E-mail address: bsm01@twntku10

Department of Mathematics, University of Wisconsin, Madison, Wisconsin 53706 E-mail address: has@math.wisc.edu 